\title{
Long-Range Reactivity Modulations in Geranyl Chloride Derivatives
}

\author{
Michael B. Reardon, Muyun Xu, Qingzhe Tan, P. George Baumgartel, Danielle J. Augur, \\ Shuanghong Huo, Charles E. Jakobsche* \\ Carlson School of Chemistry \& Biochemistry, Clark University, Worcester, MA \\ Email: cjakobsche@clarku.edu
}

Table of Contents

1. Synthetic Procedures and Compound Data ……......................................................... S2

1.1. Abbreviations ………………………………..................................... S2

1.2. General Procedures …………………………........................................... S2

1.3. Synthetic Scheme for Chloride 24 .................................................................. S3

1.4. Representative NMR Data from Kinetics Experiments .................................. S4

1.5. Results of Rate Comparisons ...................................................................... S11

2. Computational Methods ……………............................................................... S15

2.1. Conformational Analysis of Geranyl Chloride ............................................... S15

2.2. Analysis of Reactants ..................................................................................... S S15

2.3. Reactant Orbital Structures ........................................................................ S16

2.4. Analysis of Transition States ..................................................................... S17

2.5. Transition-State Orbital Structures ………………........................................... S $\mathrm{S} 18$

3. NMR Spectra from Synthesis .............................................................................. S20

4. Atomic Coordinates for Calculated Structures .......................................................... S56

4.1. Conformations of Geranyl Chloride Reactant ................................................ S56

4.2. Refined Reactant Geometries ……….......................................................... S66

4.3. Refined Transition-State Geometries ............................................................... S6 $\quad$ S6 


\section{Synthetic Procedures and Compound Data}

\subsection{Abbreviations}

$\mathrm{AcOH}=$ acetic acid

$\mathrm{DCM}=$ dichloromethane

$$
\begin{aligned}
& \mathrm{MeOH}=\text { methanol } \\
& \text { Quant }=\text { quantitative conversion } \\
& \text { TEA }=\text { triethylamine } \\
& \text { TFA }=\text { trifluoroacetic acid } \\
& \text { THF }=\text { tetrahydrofuran } \\
& \mathrm{y}=\text { yield }
\end{aligned}
$$

\subsection{General Procedures}

Column chromatography was performed with $60 \AA 40-63 \mu \mathrm{m}$ silia-P flash silica gel.

Solvents for reactions (DMF, DCM, THF, and toluene) were dried using a LC Technology Solutions purification system. Other solvents were used as received unless noted otherwise.

Purchased Chemicals were used as received unless noted otherwise.

NMR Spectra were measured in $\mathrm{CDCl}_{3}$ at ambient temperature unless otherwise noted.

${ }^{1}$ H NMR spectra were recorded on either a 600 or $200 \mathrm{MHz}$ spectrometer. Chemical shifts are reported in ppm $(\delta)$ relative to tetramethylsilane using the solvent as a reference $\left(\mathrm{CDCl}_{3}=7.26 \mathrm{ppm}\right.$, DMSO- $d_{6}=2.49 \mathrm{ppm}, \mathrm{D}_{2} \mathrm{O}=$ $4.80 \mathrm{ppm}, \mathrm{CD}_{3} \mathrm{OD}=3.30$ ). The following is an example data point: chemical shift (multiplicity [s = singlet, $\mathrm{d}=$ doublet, $\mathrm{t}=$ triplet, $\mathrm{q}=$ quartet, pent $=$ pentet, $\mathrm{sext}=$ sextet, $\mathrm{sept}=$ septet, oct $=$ octet, $\mathrm{m}=$ multiplet, $\mathrm{br}=$ broad, and combinations thereof], coupling constants [Hz], integration, assignment [if any]).

${ }^{13}$ C NMR spectra were recorded on either a 600 or $200 \mathrm{MHz}(150$ or $50 \mathrm{MHz})$ spectrometer with complete proton decoupling. Chemical shifts are reported in ppm $(\delta)$ relative to tetramethylsilane using the solvent or $\mathrm{MeOH}$ as a reference $\left(\mathrm{CDCl}_{3}=77.16 \mathrm{ppm}, \mathrm{DMSO}-d_{6}=39.52 \mathrm{ppm}, \mathrm{CD}_{3} \mathrm{OD}=49.00 \mathrm{ppm}, \mathrm{MeOH}=49.5\right)$.

IR spectra are partially reported $\left(v_{\max }, \mathrm{cm}^{-1}\right)$.

MS were obtained using quadrupole analyzers..

TLC was performed on $60 \AA \mathrm{F}_{254}$ pre-coated silica gel plates. Samples were visualized by either ultraviolet irradiation, potassium permanganate staining, or cerium ammonium molybdate staining.

Yield refers to isolated material.

Quantitative recovery means that mostly pure material was recovered in approximately the expected mass, and the material was used directly for the next step without purification. 


\subsection{Synthetic Scheme for Chloride 24}

Scheme S1 outlines the synthesis of chloride 24 .
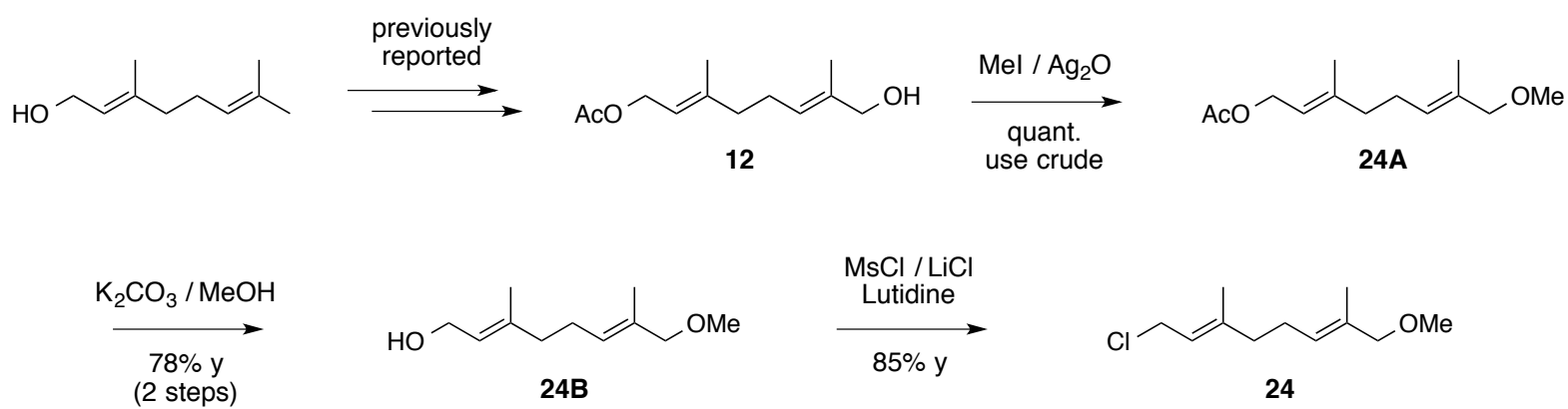

Scheme S1. Synthetic scheme for accessing chloride 24. 


\subsection{Representative NMR Data from Kinetics Experiments}

Reaction of Chloride 3 into Pyridinium 9

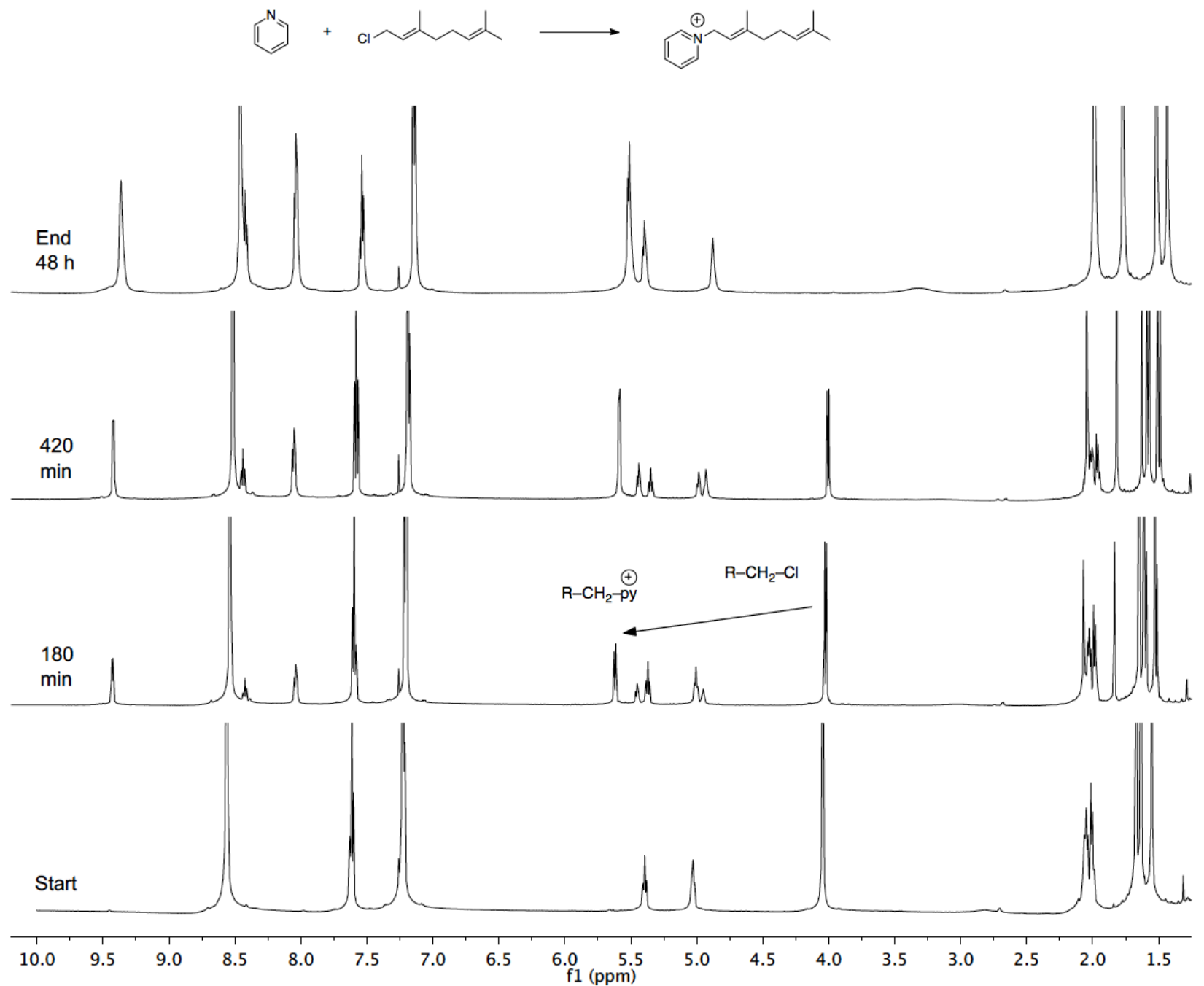


Reaction of Chloride 4 into Pyridinium 10

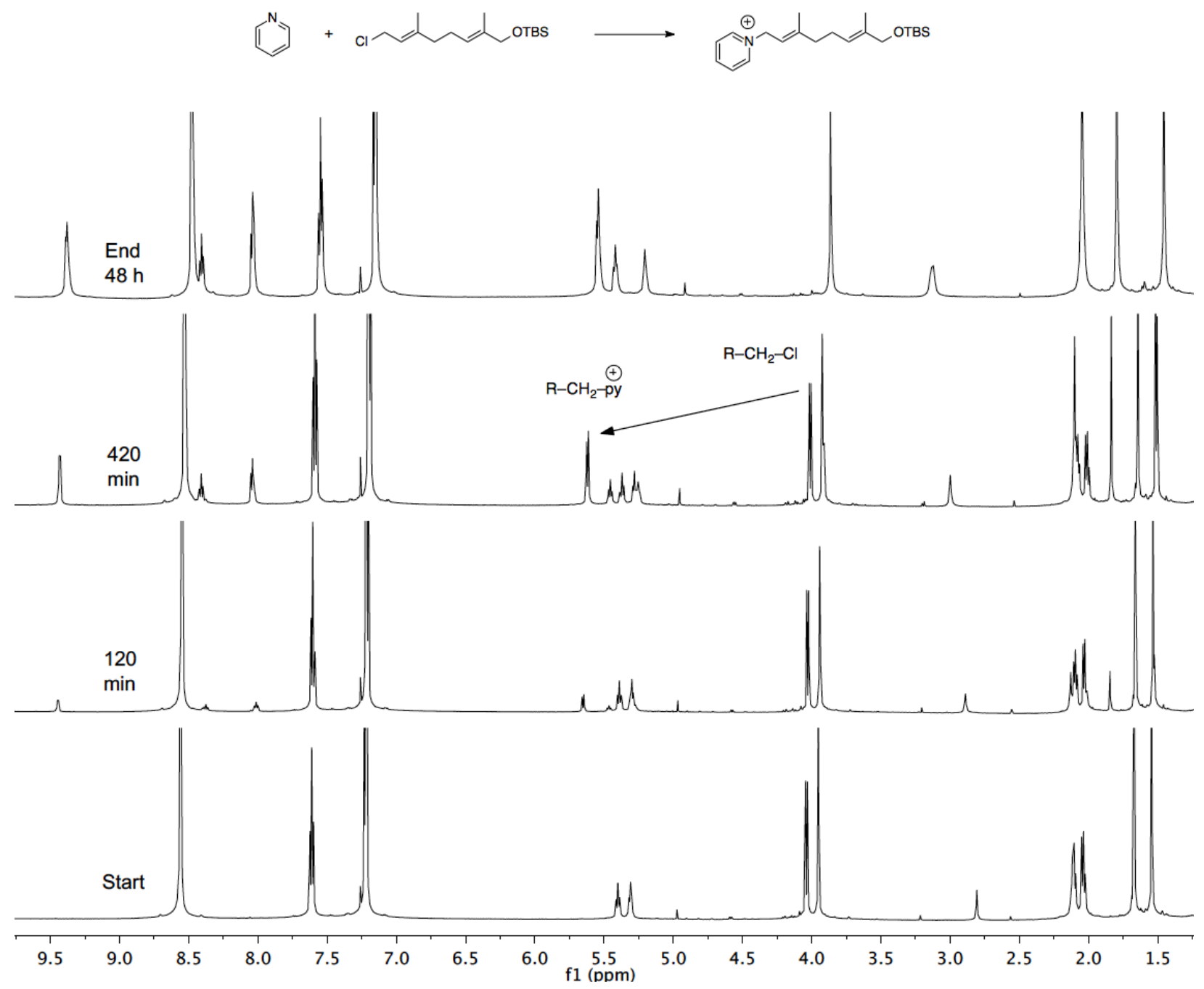




\section{Reaction of Chloride 5 into Pyridinium 11}

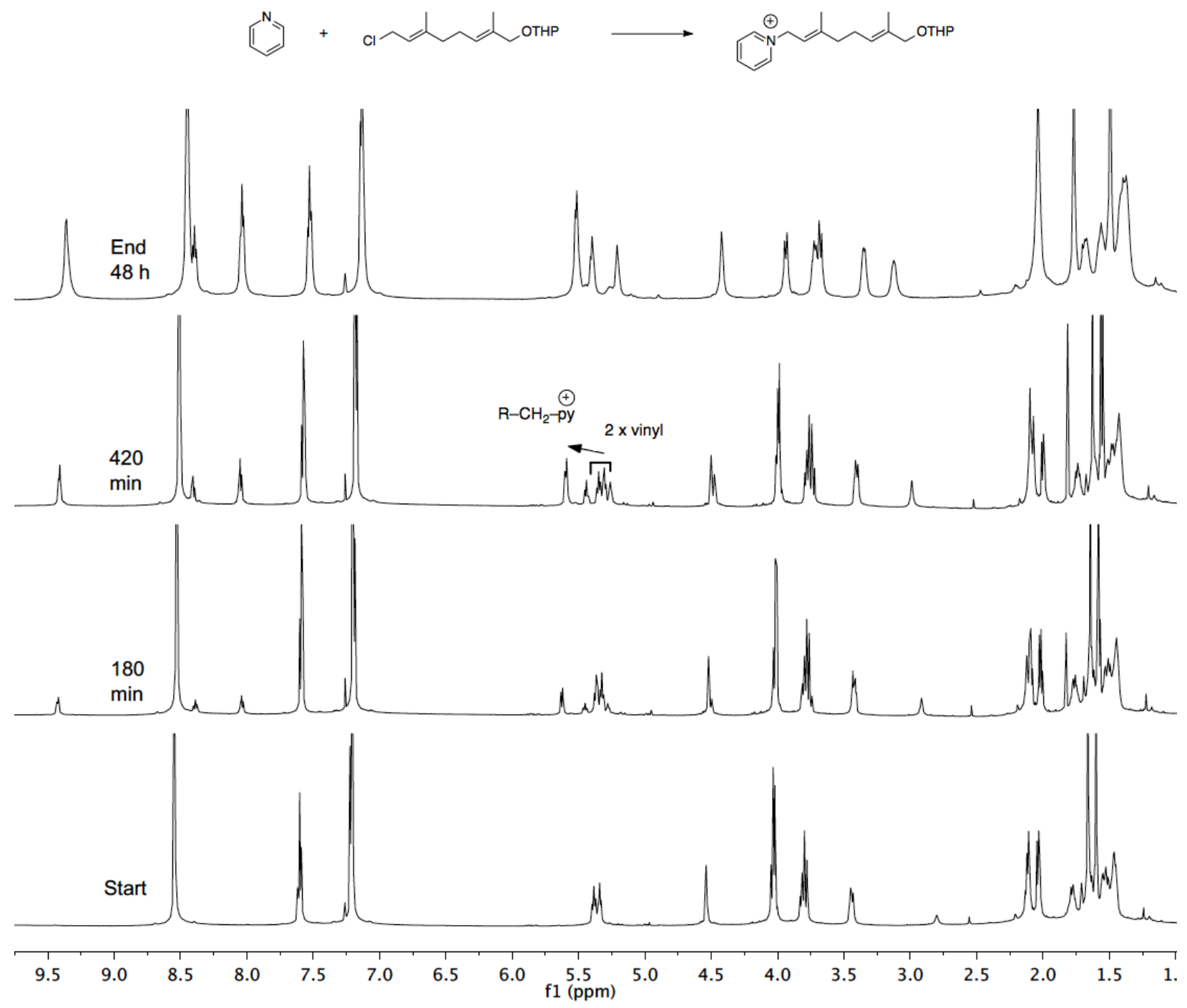




\section{Reaction of Chloride 16 into Pyridinium 16A}
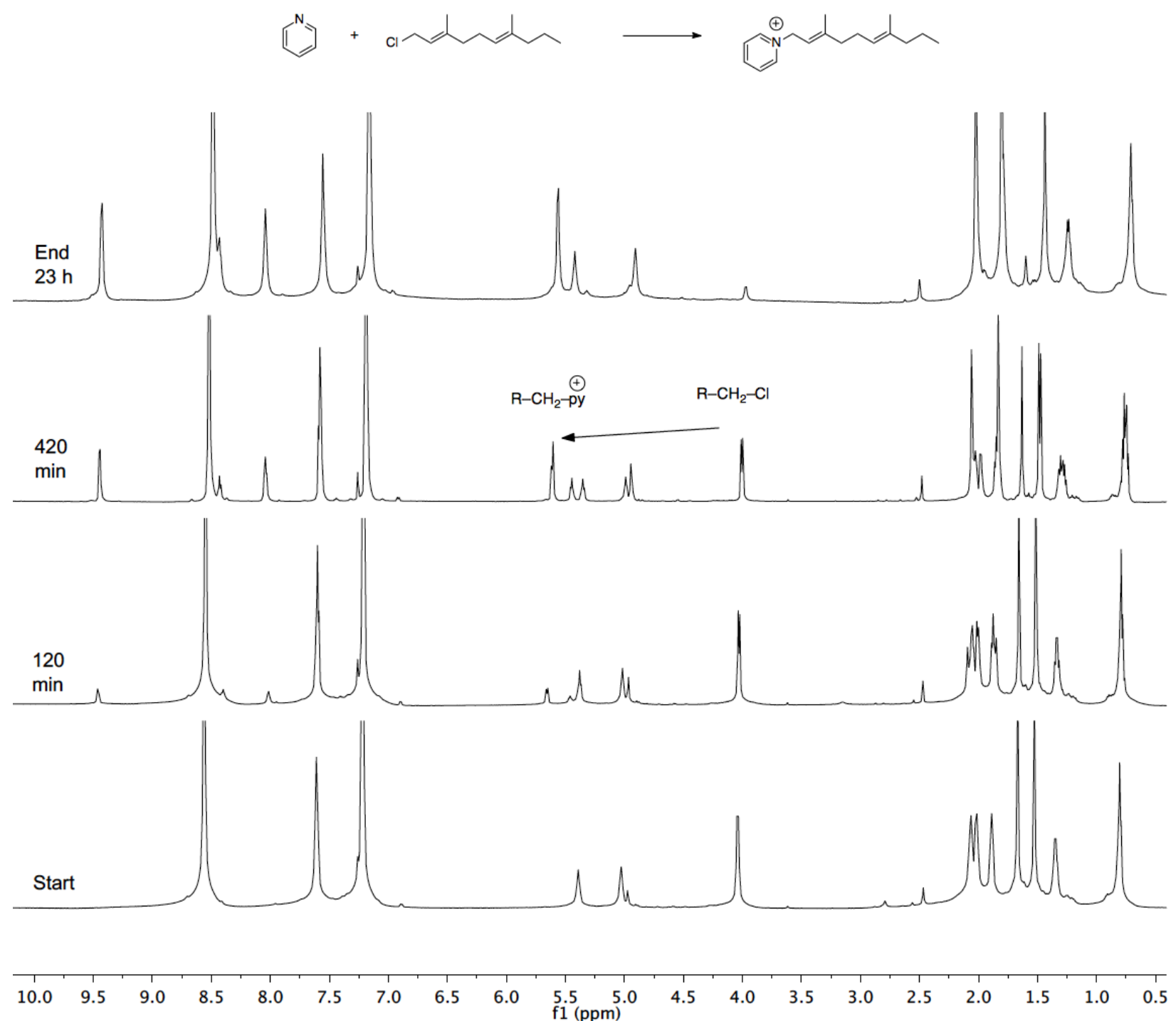
Reaction of Chloride 22 into Pyridinium 22A

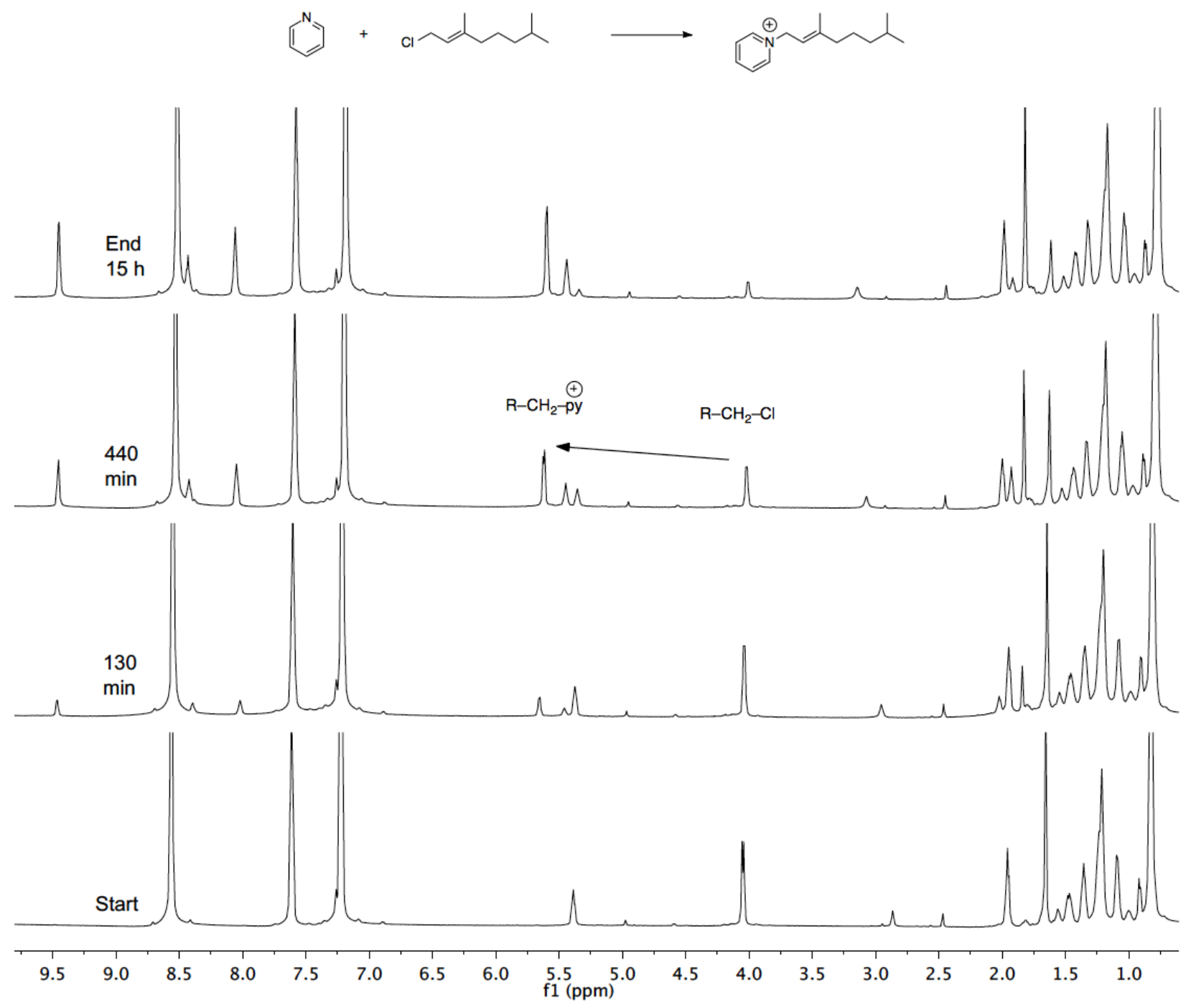




\section{Reaction of Chloride 23 into Pyridinium 23A}

The prenyl chloride contained approximately $10 \%$ of the isomeric 3-methyl-3chloro-1-butene, which did not react during the course of these experiments.

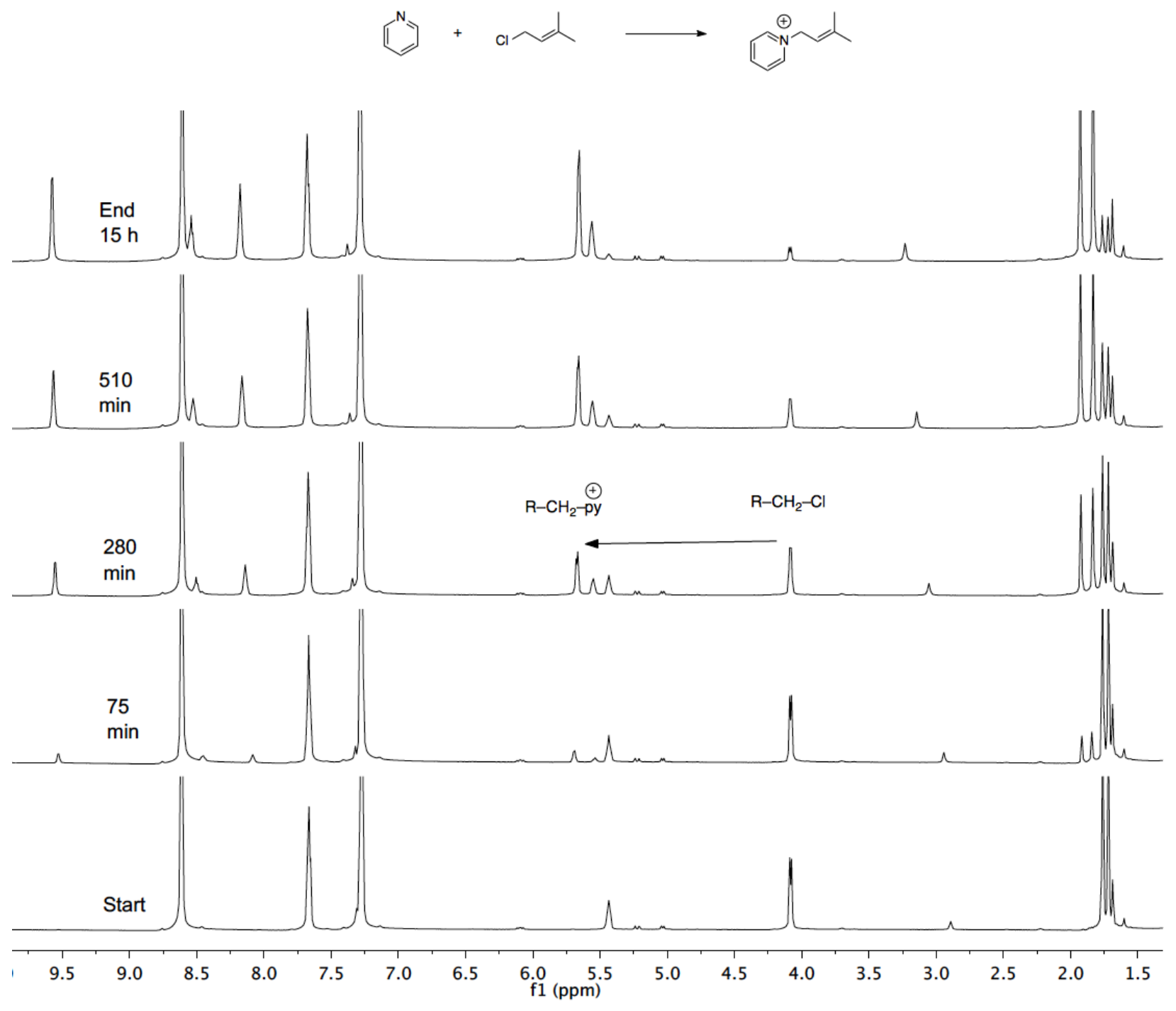




\section{Reaction of Chloride 24 into Pyridinium 24C}

Chloride $\mathbf{2 4}$ contained approximately $5 \%$ of the isomeric tertiary chloride, which did not react during the course of these experiments.

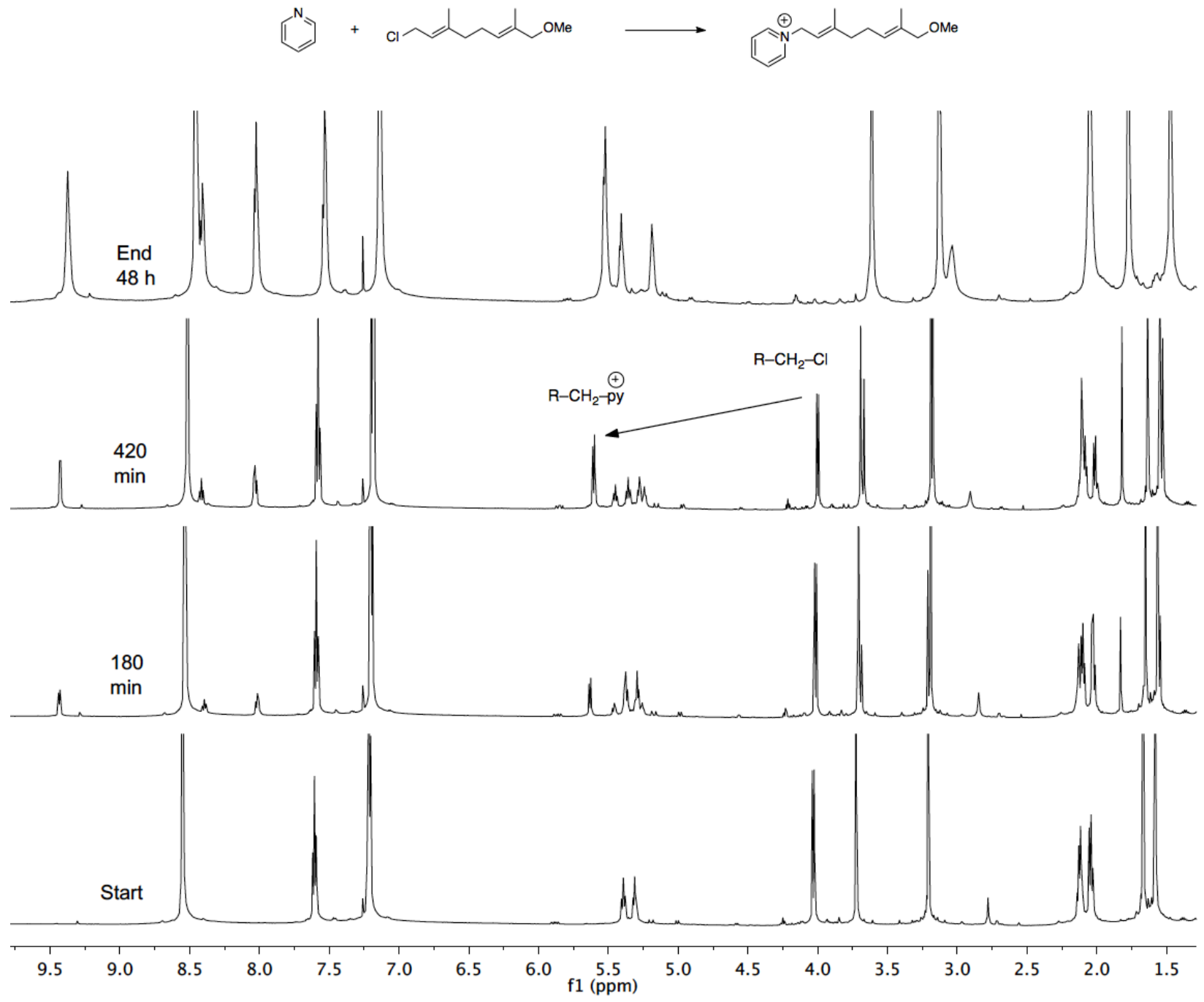




\subsection{Results of Rate Comparisons}

Runs \#1 and \#2 compare geranyl chloride (3) to tert-butyldimethylsilyl-protected hydroxygeranyl chloride 4 and tetrahydropyranyl-ptotected hydroxygeranyl chloride 5. Runs \#3 and \#4 compare geranyl chloride (3) to prenyl chloride (23) and monounsaturatedgeranylchloride 22. Runs \#5 and \#6 compare geranyl chloride (3) to ethylgeranyl chloride 16 and methoxygeranylchloride 24. All experiments were run at $23-24{ }^{\circ} \mathrm{C}$, and each experiment was maintained in a $+/-0.5{ }^{\circ} \mathrm{C}$ range and compared to other runs performed simultaneously at the same temperature. Each separate graph represents data that were collected from experiments that were run in side-by-side experiments. Lines of best fit, which were forced through the origin, were calculated using Microsoft Excel 14.3.9. Slopes were compared against others from within the same run, and then the results from replicate runs were averaged. Figures S1-S7 show these data. To show that these rate differences are maintained over longer time periods, data was collected from a representative experiment over 24 hours, during which time $>95 \%$ of the geranyl chloride reacted. Figure S8 shows these data.

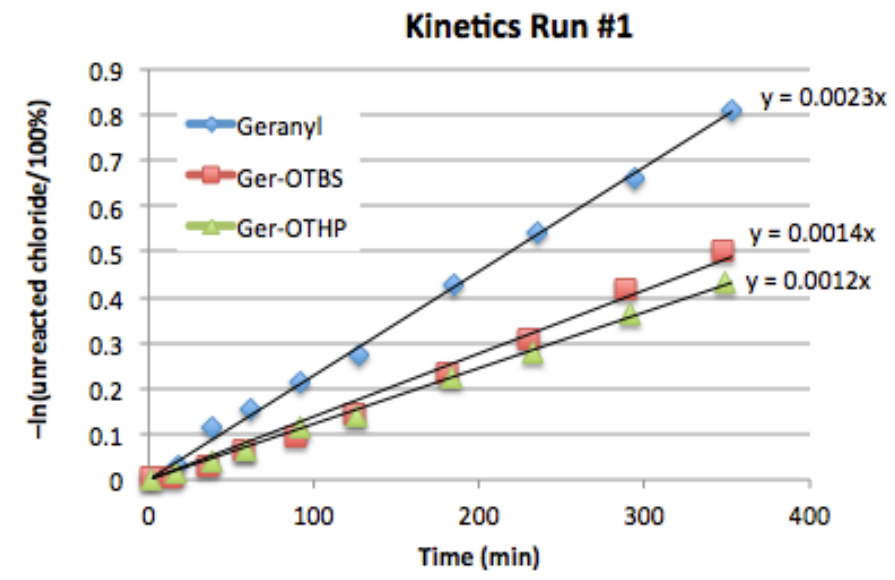

Figure S1. Data from Kinetics Run \#1. Temperature $=24{ }^{\circ} \mathrm{C}+/-0.5{ }^{\circ} \mathrm{C}$. Ger-OTBS $=41 \%$ decrease versus geranyl. Ger-OTHP $=45 \%$ decrease versus geranyl.

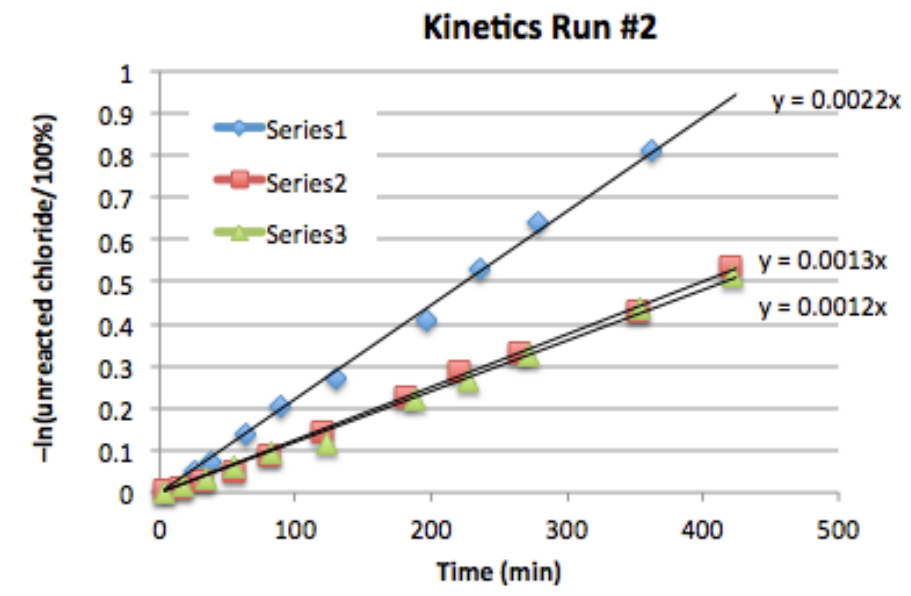

Figure S2. Data from Kinetics Run \#2. Temperature $=24{ }^{\circ} \mathrm{C}+/-0.5{ }^{\circ} \mathrm{C}$. Ger-OTBS $=39 \%$ decrease versus geranyl. Ger-OTHP $=48 \%$ decrease versus geranyl.

\section{Averages of runs \#1 and \#2}

Ger-OTBS $=40 \%$ decrease from geranyl, standard deviation $=1.3 \%$

Ger-OTHP $=47 \%$ decrease from geranyl, standard deviation $=1.7 \%$ 


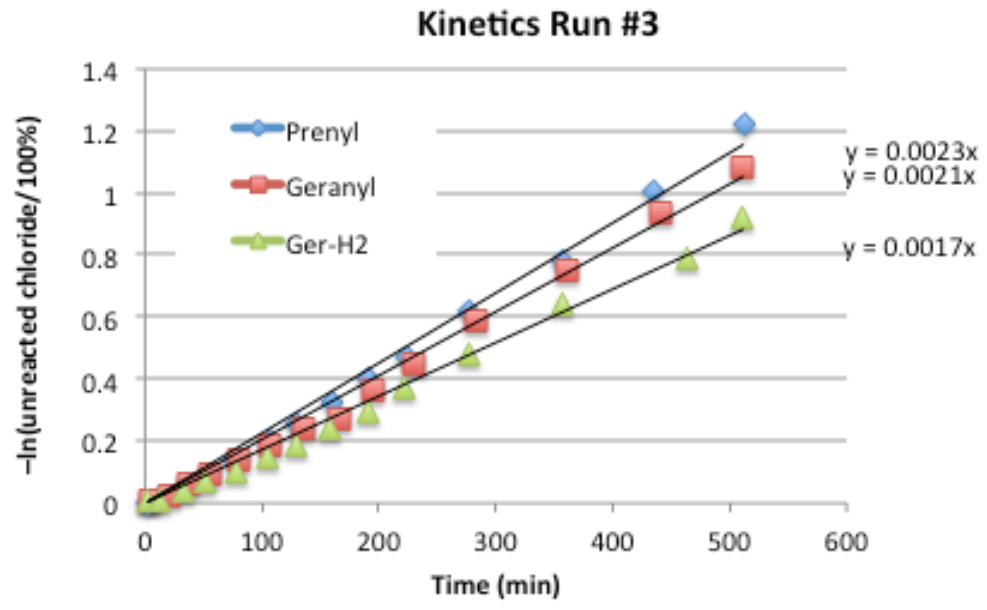

Figure S3. Data from Kinetics Run \#3. Temperature $=24{ }^{\circ} \mathrm{C}+/-0.5^{\circ} \mathrm{C}$. Ger- $\mathrm{H}_{2}=19 \%$ decrease versus geranyl. Prenyl $=10 \%$ increase versus geranyl.

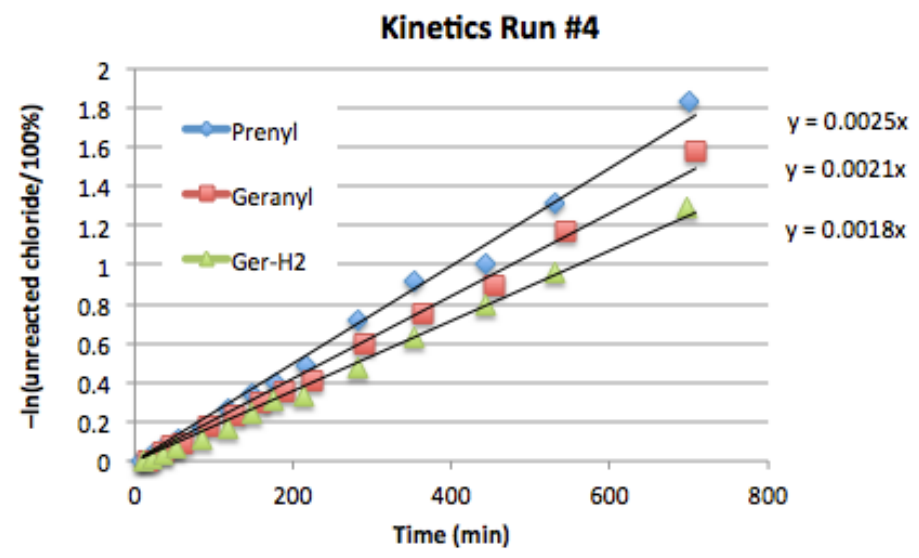

Figure S4. Data from Kinetics Run \#4. Temperature $=24{ }^{\circ} \mathrm{C}+/-0.5^{\circ} \mathrm{C} . \mathrm{Ger}-\mathrm{H}_{2}=14 \%$ decrease versus geranyl. Prenyl $=19 \%$ increase versus geranyl.

\section{Averages of runs \#3 and \#4}

Ger- $\mathrm{H}_{2}=17 \%$ decrease from geranyl, standard deviation $=3.4 \%$

Prenyl $=14 \%$ increase from geranyl, standard deviation $=6.7 \%$ 


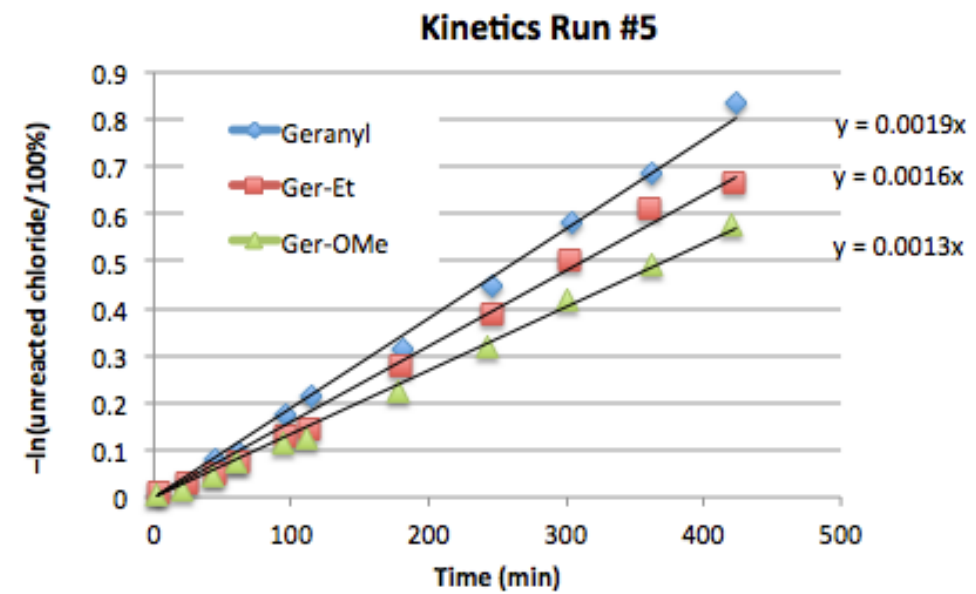

Figure S5. Data from Kinetics Run $\# 5$. Temperature $=24{ }^{\circ} \mathrm{C}+/-0.5{ }^{\circ} \mathrm{C}$. Ger-Et $=16 \%$ decrease versus geranyl. Ger-OMe $=32 \%$ decrease versus geranyl.

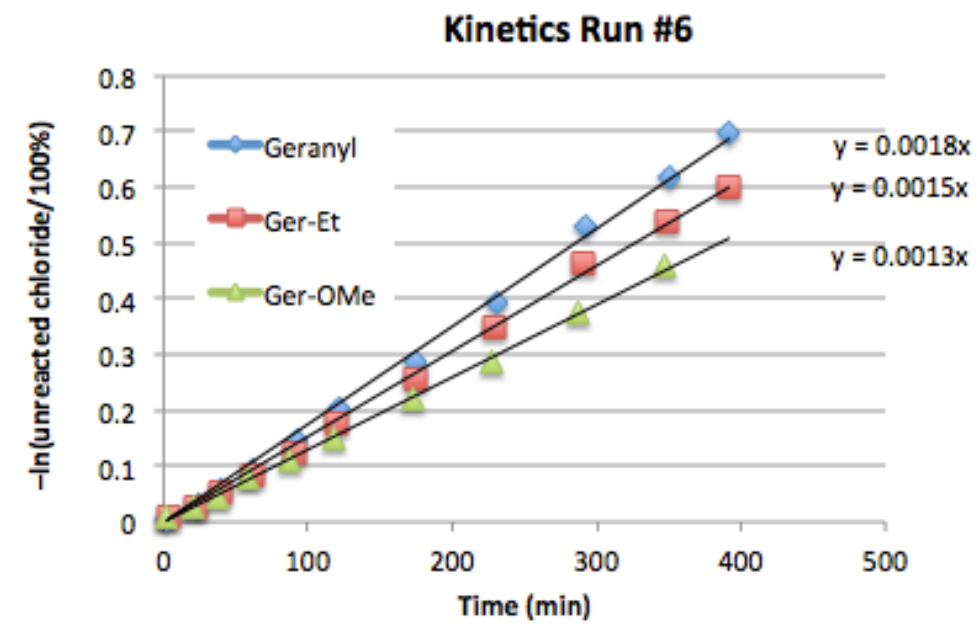

Figure S6. Data from Kinetics Run \#6. Temperature $=23{ }^{\circ} \mathrm{C}+/-0.5{ }^{\circ} \mathrm{C}$. Ger-Et $=17 \%$ decrease versus geranyl. Ger-OMe $=28 \%$ decrease versus geranyl.

\section{Averages of runs \#5 and \#6}

Ger-Et $=16 \%$ decrease from geranyl, standard deviation $=0.6 \%$

Ger-OMe $=30 \%$ decrease from geranyl, standard deviation $=2.7 \%$ 


\section{Relative Reaction Rates}

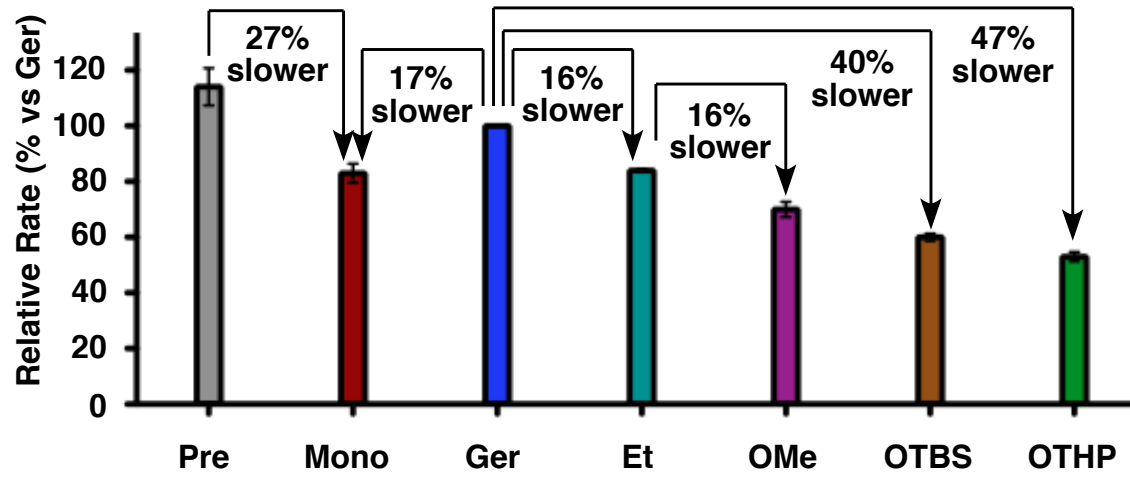

Figure S7. Summary of relative rates of reaction of geranyl chloride analogues with pyridine. Pre $=$ prenyl chloride (23); Mono = monounsaturated geranyl chloride 22; Ger = geranyl chloride (3); Et = ethylgeranyl chloride 16; $\mathrm{OMe}=$ methoxygeranyl chlorided 24; OTBS = tertbutyldimethylsiloxygeranyl chloride 4; OTHP = tetrahydropyranyloxygeranyl chloride 5. Error bars represent standard deviations of duplicate experiments.

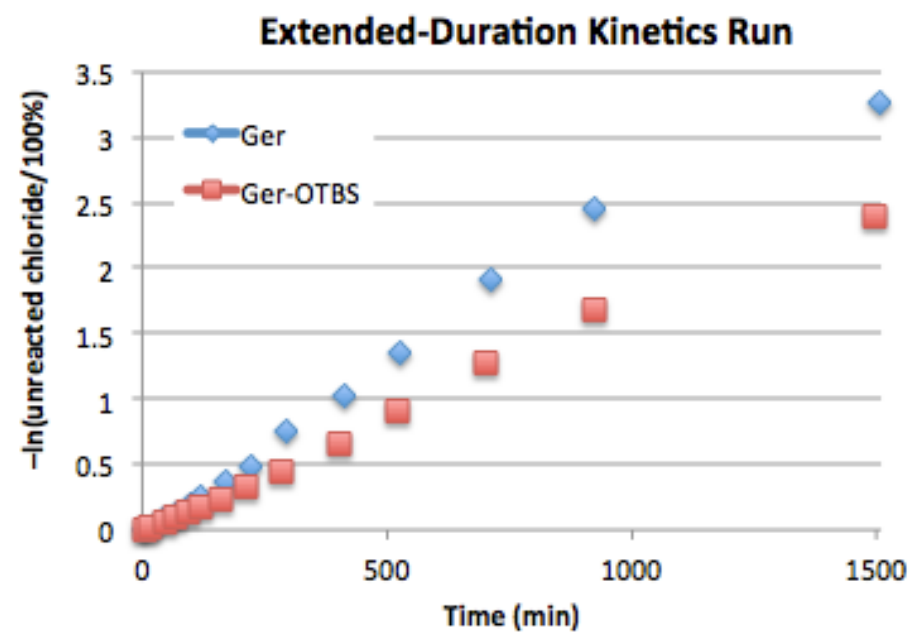

Figure S8. A representative long-time-scale kinetics experiment comparing geranyl chloride to OTBSfunctionalized geranyl chloride 4 .. Temperature $=28{ }^{\circ} \mathrm{C}+/-0.5^{\circ} \mathrm{C}$. These data were not used in rate comparisons, but simply show that the difference in reaction rates are maintained over longer periods of time and eventually diverge from linearity at very high conversion (as the pyridine concentration becomes reduced). 


\section{Computational Methods}

References for software packages and methods are listed in the body of the manuscript.

\subsection{Conformational Analysis of Geranyl Chloride}

Conformational options for geranyl chloride were identified with Molecular Operating Environment 2015.10 using the LowModeMD method (rejection limit $=100$, iteration limit $=10,000$, RMS gradient $=0.005$, MM iteration limit $=500$, RMSD limit $=0.25$, conformation limit $=10,000)$ with the Amber10:EHT forcefield. Twenty seven conformations were identified, including thirteen pairs of enantiomers. For each of the fourteen non-enantiomeric conformations, energy levels were calculated using the Amber10:EHT forcefield (Table S1). Atomic coordinates for these conformations are listed in Section 4, below. To create Figure 6, these fourteen structures were refined using Gaussian 09 with the B3LYP functional and the 6-311g(d) basis set with the PCM solvation model for dichloromethane, and then these structures were overlapped using UCSF Chimera 1.10.2.

Table S1. Geranyl Chloride conformations and energies

\begin{tabular}{|c|c|c|}
\hline Conformation \# & $\begin{array}{c}\text { Free Energy (kcal/mol) } \\
\text { of Preliminary Structure }\end{array}$ & Nickname for Selected Conformations \\
\hline 1 & 0.9049 & \\
\hline 2 & 0.9071 & Down \\
\hline 3 & 1.0703 & \\
\hline 4 & 1.1299 & Up \\
\hline 5 & 2.5672 & \\
\hline 6 & 2.9226 & Far Down \\
\hline 8 & 2.9302 & Far Up \\
\hline 9 & 2.9344 & \\
\hline 10 & 3.0278 & \\
\hline 11 & 3.0714 & \\
\hline 12 & 3.5320 & \\
\hline 13 & 3.6117 & \\
\hline
\end{tabular}

\subsection{Analysis of Reactants}

For prenyl chloride, four of the conformations of geranyl chloride (far up, up, down, and far down), and pyridine, each structure was energy minimized using Gaussian 09 with the B3LYP functional and the 6-311g(d) basis set with the PCM solvation model for dichloromethane. Energies for these structures were calculated using the M06-2X method with the 6-31+g(d,p) basis set and the PCM solvation model for dichloromethane. Energy levels for each of these structures are listed in Table S2. Atomic coordinates are listed in Section 4, below.

Table S2. Refined Reactant Structures.

\begin{tabular}{|c|c|c|c|c|}
\hline Compound / Conformation & $\begin{array}{c}\text { Free Energy } \\
(\text { Hartree })\end{array}$ & $\begin{array}{c}\text { LUMO Energy } \\
(\mathrm{eV})\end{array}$ & $\begin{array}{c}\text { HOMO Energy } \\
(\mathrm{eV})\end{array}$ & $\begin{array}{c}\text { HOMO-1 Energy } \\
(\mathrm{eV})\end{array}$ \\
\hline Prenyl chloride & -655.920113 & 0.00277 & -0.30676 & -0.36288 \\
\hline Geranyl chloride far up & -851.068847 & -0.00011 & -0.29006 & -0.30603 \\
\hline Geranyl chloride up & -851.066460 & -0.00088 & -0.28896 & -0.30849 \\
\hline Geranyl chloride down & -851.066538 & 0.00132 & -0.28847 & -0.31112 \\
\hline Geranyl chloride far down & -851.070889 & 0.00039 & -0.29135 & -0.30586 \\
\hline Pyridine & -248.128941 & --- & --- & --- \\
\hline
\end{tabular}




\subsection{Reactant Orbital Structures}

Figures S9-S11 show the orbitals calculated for each of the five reactant structures. Orbitals were visualized using Gauss View at isovalue $=0.02$.

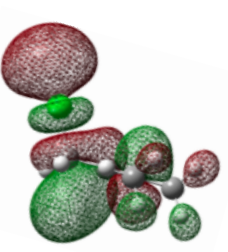

prenyl

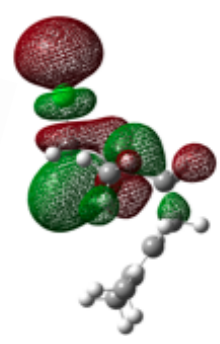

far down

\section{Reactant LUMO Structures}

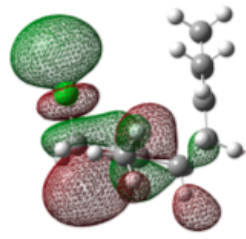

far up

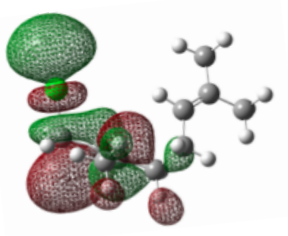

up

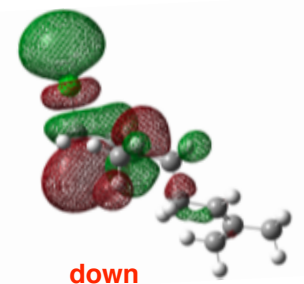

down
Figure S9. Structures of reactant LUMOs. prenyl

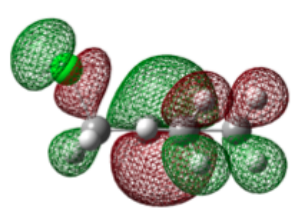

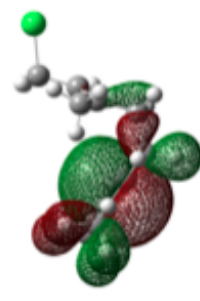

far down
Reactant HOMO Structures
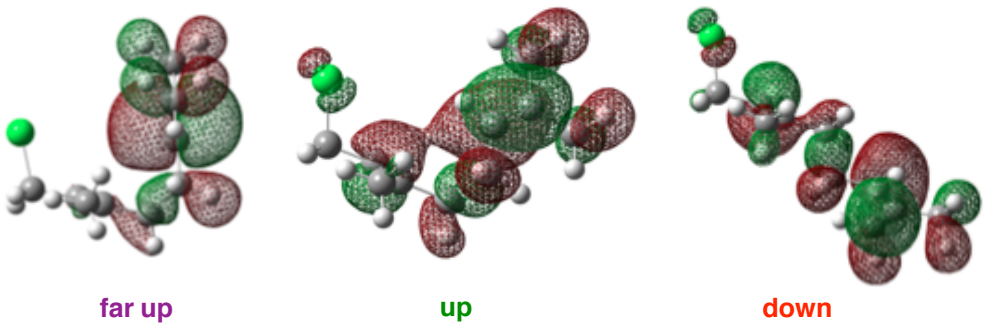

Figure S10. Structures of reactant HOMOs.

Reactant HOMO-1 Structures
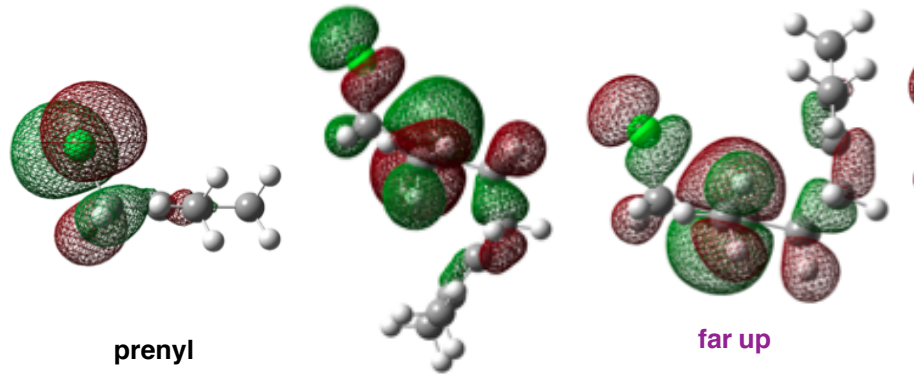

far up

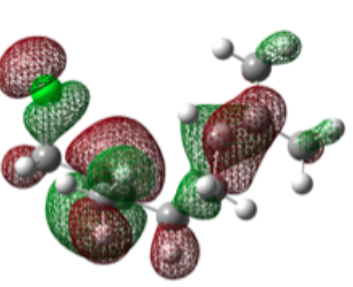

up

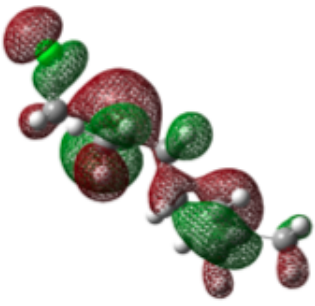

down

Figure S11. Structures of reactant HOMO-1s. 


\subsection{Analysis of Transition States}

For each of the five starting materials described above, a transition-state structure was found using Gaussian 09 using the Berny method with the B3LYP functional and the 6-311g(d) basis set with the PCM solvation model for dichloromethane. IRC calculations using the same level of theory confirmed that each transition state did indeed connect the reactant to the expected product. Energies for these transition state structures were calculated using the M06-2X method with the 6-31+g(d,p) basis set and the PCM solvation model for dichloromethane. Energy levels for each of these structures are listed in Table S3. Atomic coordinates are listed in Section 4, below.

Table S3. Transition-State Structures

\begin{tabular}{|c|c|c|c|c|}
\hline Compound / Conformation & $\begin{array}{c}\text { Free Energy } \\
(\text { Hartree })\end{array}$ & $\begin{array}{c}\text { LUMO Energy } \\
(\mathrm{eV})\end{array}$ & $\begin{array}{c}\text { HOMO Energy } \\
(\mathrm{eV})\end{array}$ & $\begin{array}{c}\text { HOMO-1 Energy } \\
(\mathrm{eV})\end{array}$ \\
\hline Prenyl chloride & -904.010738 & -0.05697 & -0.29743 & -0.31046 \\
\hline Geranyl chloride far up & -1099.153436 & -0.05919 & -0.29033 & -0.29750 \\
\hline Geranyl chloride up & -1099.155606 & -0.05901 & -0.28955 & -0.29969 \\
\hline Geranyl chloride down & -1099.152849 & -0.06055 & -0.29117 & -0.30224 \\
\hline Geranyl chloride far down & -1099.152843 & -0.05905 & -0.29529 & -0.29877 \\
\hline
\end{tabular}




\subsection{Transition-State Orbital Structures}

Figures S12-S17 show the orbitals calculated for each of the five transition-state structures. Orbitals were visualized using GaussView at isovalue $=0.02$.

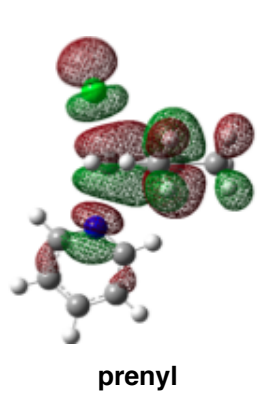

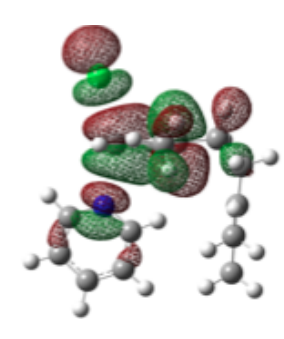

far down

Transition State LUMO Structures

Figure S12. Structures of transition-state LUMOs.

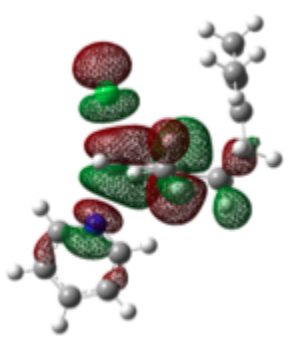

far up

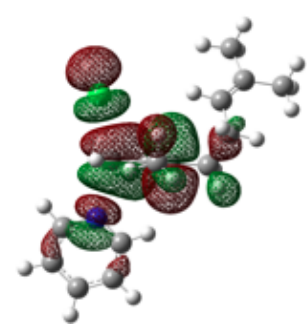

up

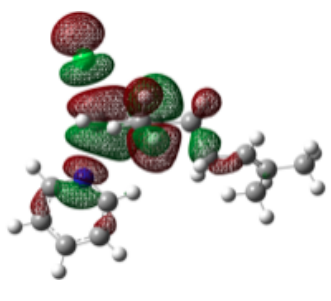

down

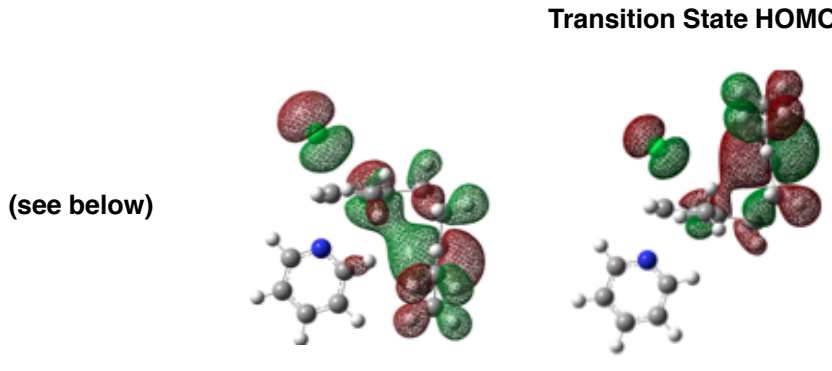

prenyl

far down

far up

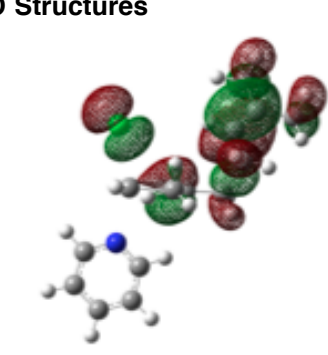

up

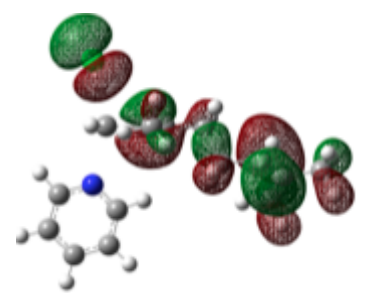

down

Figure S13. Structures of transition-state HOMOs.

Transition State HOMO-1 (Geranyl) and HOMO (Prenyl) Structures

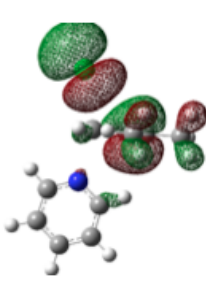

prenyl

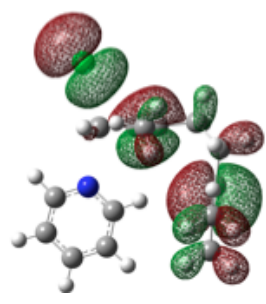

far down

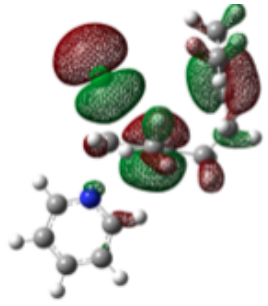

far up

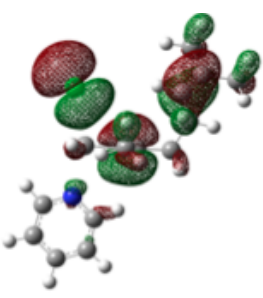

up

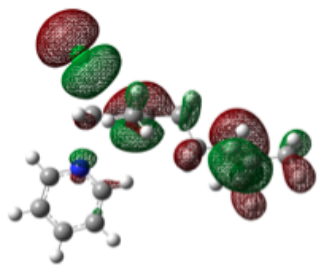

down

Figure S14. Structures of transition-state HOMO-1s and HOMO.

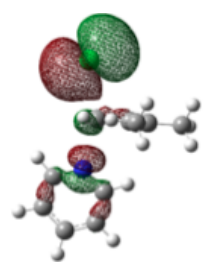

prenyl

Transition State HOMO-2 (Geranyl) and HOMO-1 (Prenyl) Structures

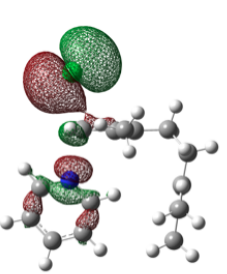

far down

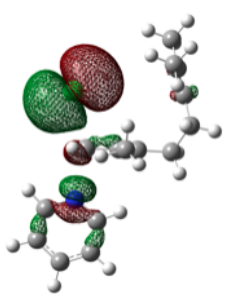

far up

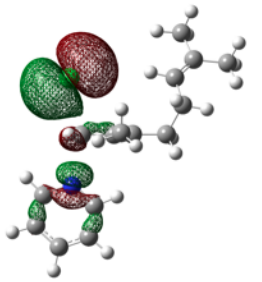

up

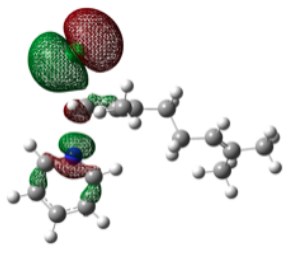

down

Figure S15. Structures of transition-state HOMO-2s and HOMO-1. 


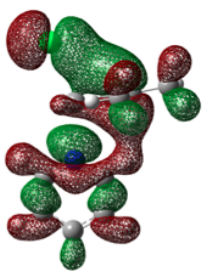

prenyl

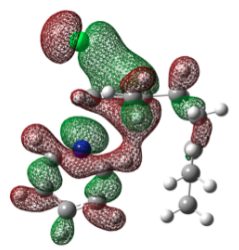

far down

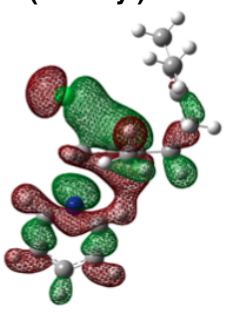

far up

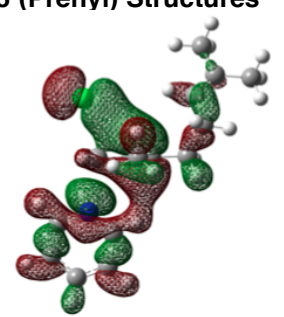

up

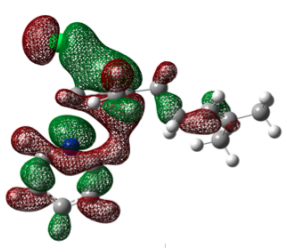

down

Figure S16. Structures of transition-state HOMO-4s and HOMO-3.

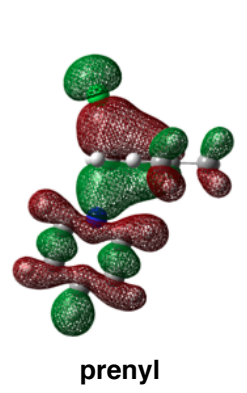

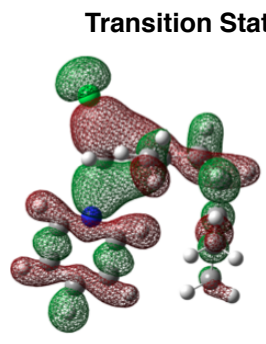

far down

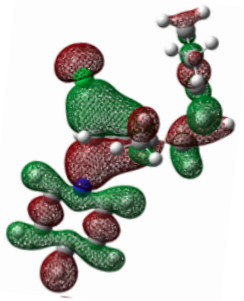

far up

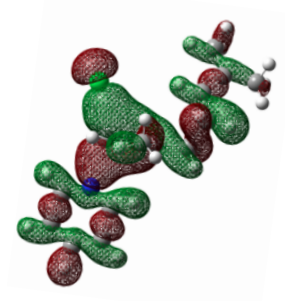

up

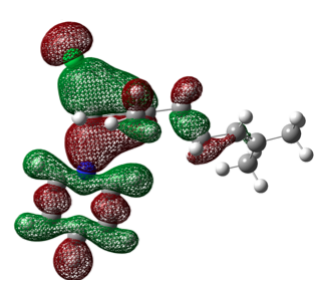

down

Figure S17. Structures of transition-state HOMO-8s and HOMO-6. These orbitals are the ones that most resemble an in-phase combination of the pyridine's lone pair orbital and the $\mathrm{C}-\mathrm{Cl}$ antibonding orbital. 
${ }^{1}$ H NMR Spectrum of 4A

\section{NMR Spectra from Synthesis}

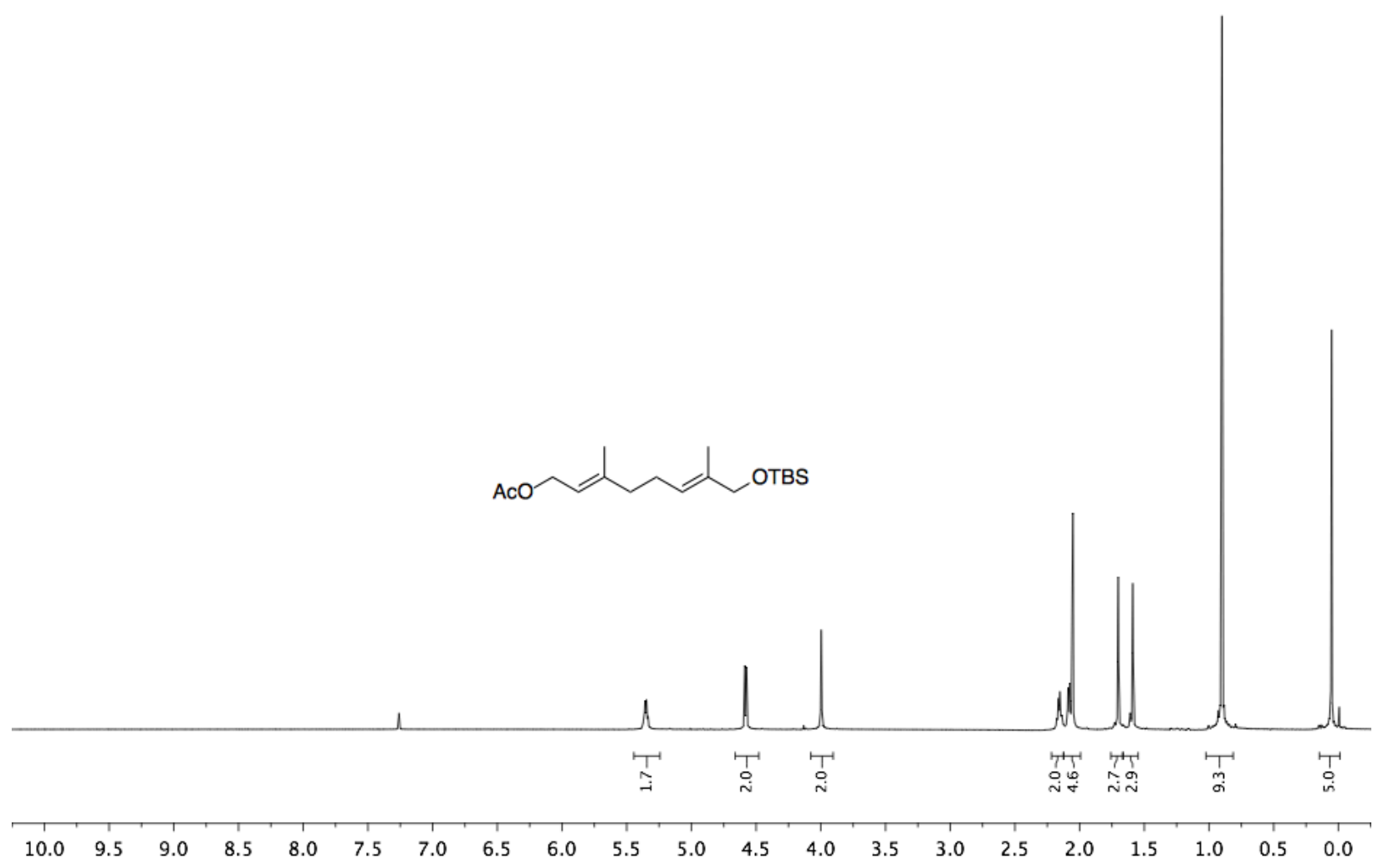

${ }^{13} \mathrm{C}$ NMR Spectrum of $4 \mathrm{~A}$
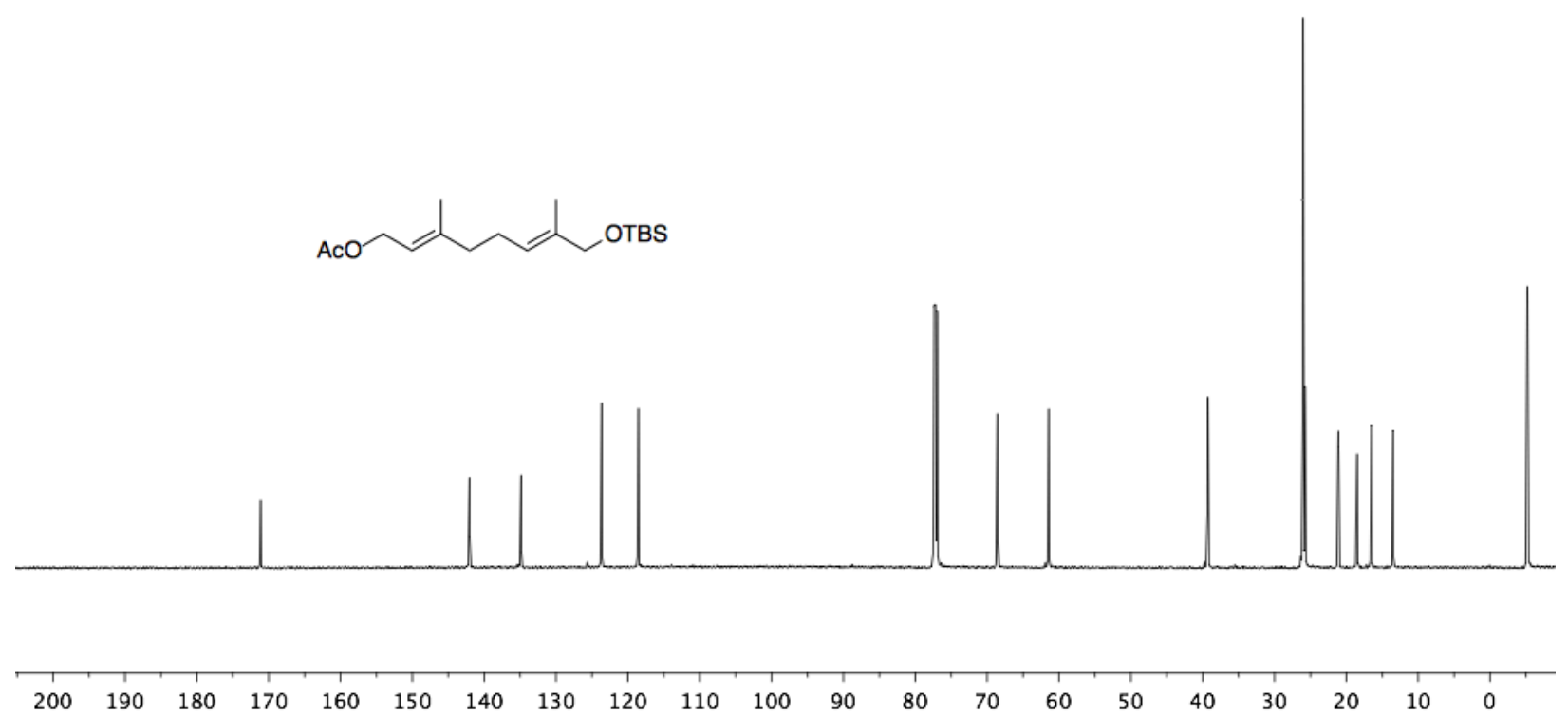


\section{${ }^{1} \mathrm{H}-{ }^{1} \mathrm{H}$ COSY NMR Spectrum of $4 \mathrm{~A}$}

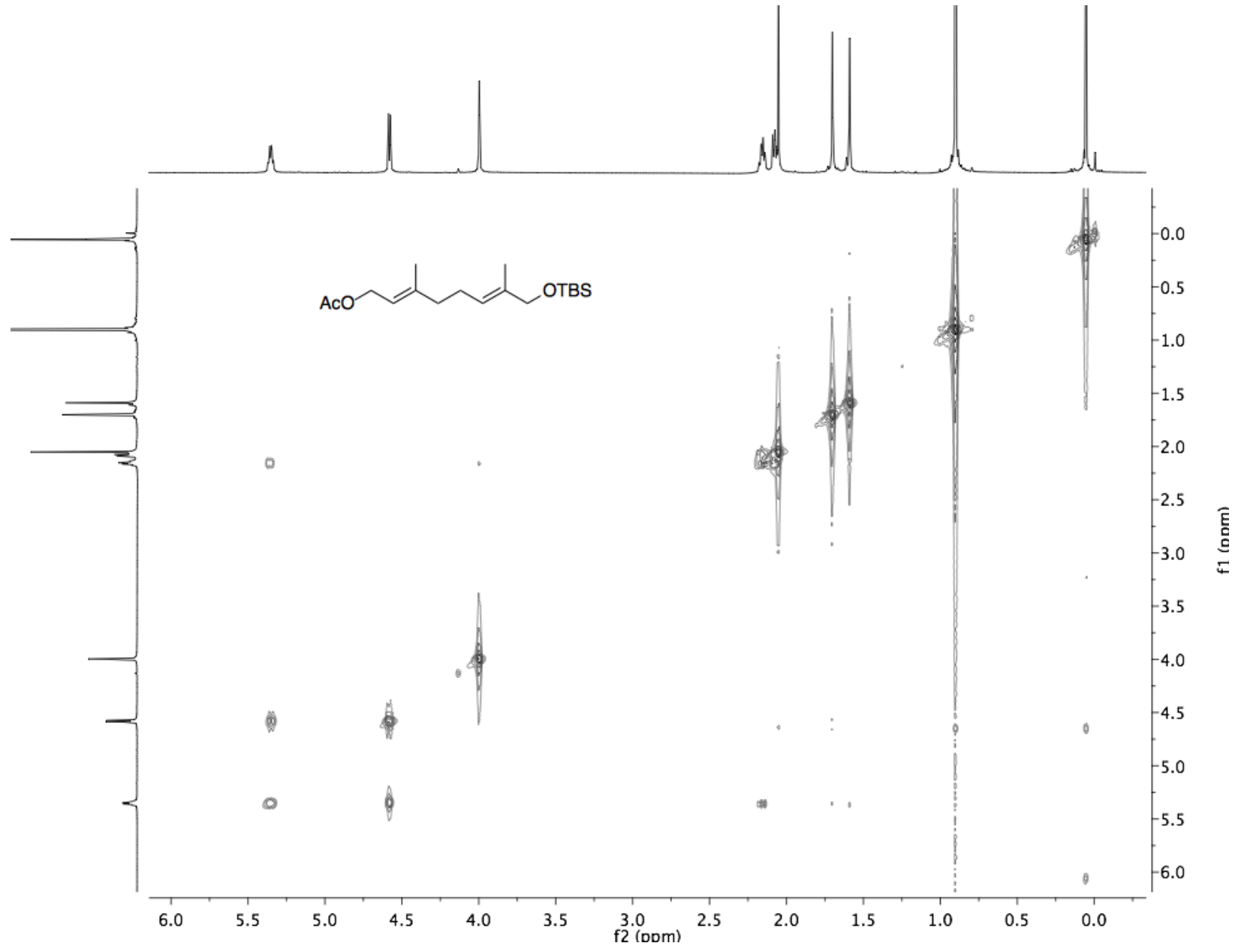




\section{${ }^{1} \mathrm{H}$ NMR Spectrum of 4B}

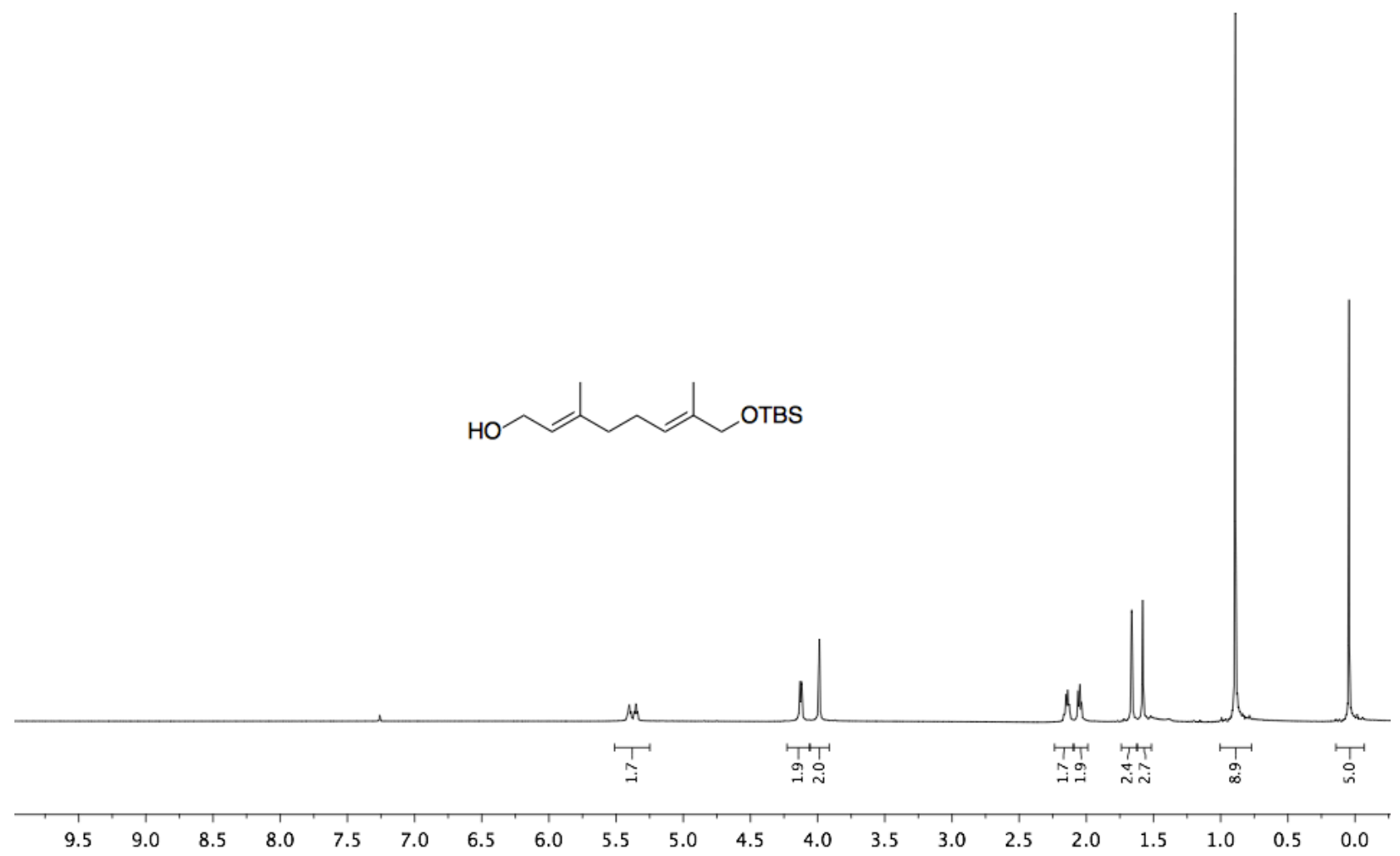

${ }^{13} \mathrm{C}$ NMR Spectrum of $4 \mathrm{~B}$

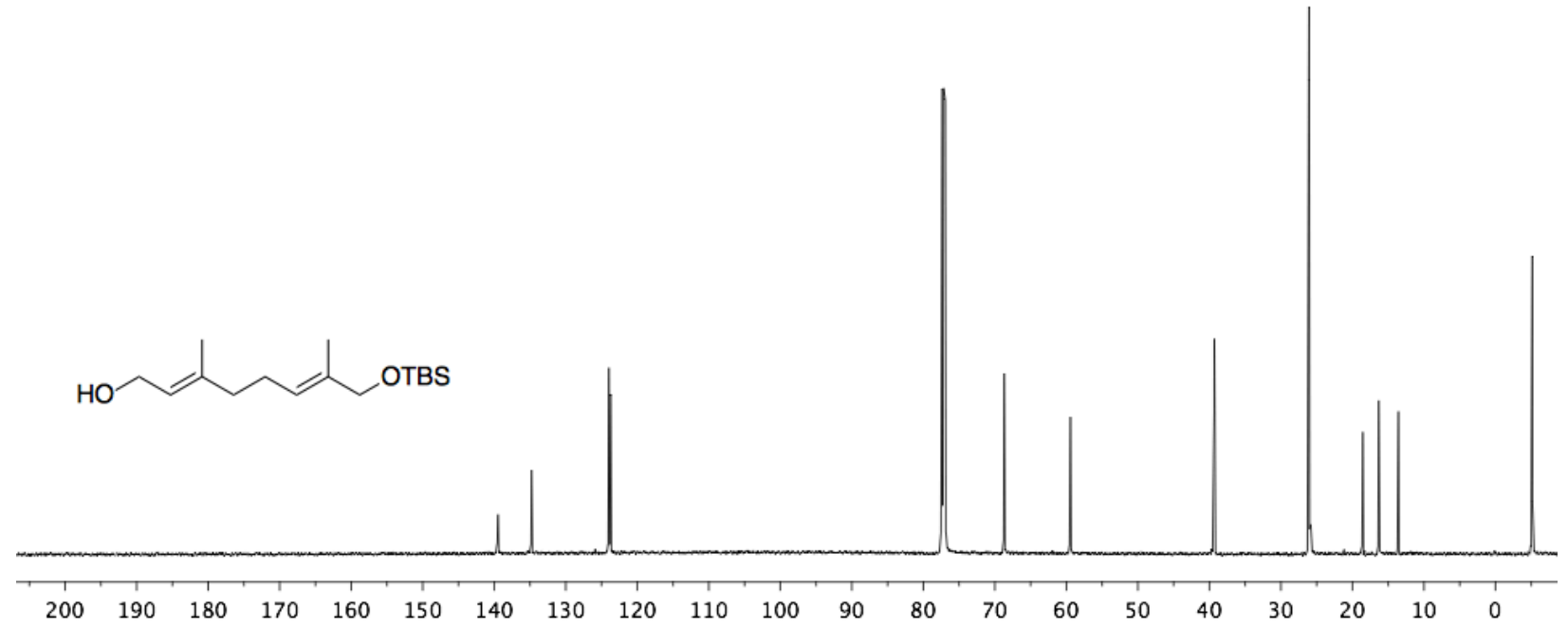




\section{${ }^{1} \mathrm{H}-{ }^{1} \mathrm{H}$ COSY NMR Spectrum of 4B}

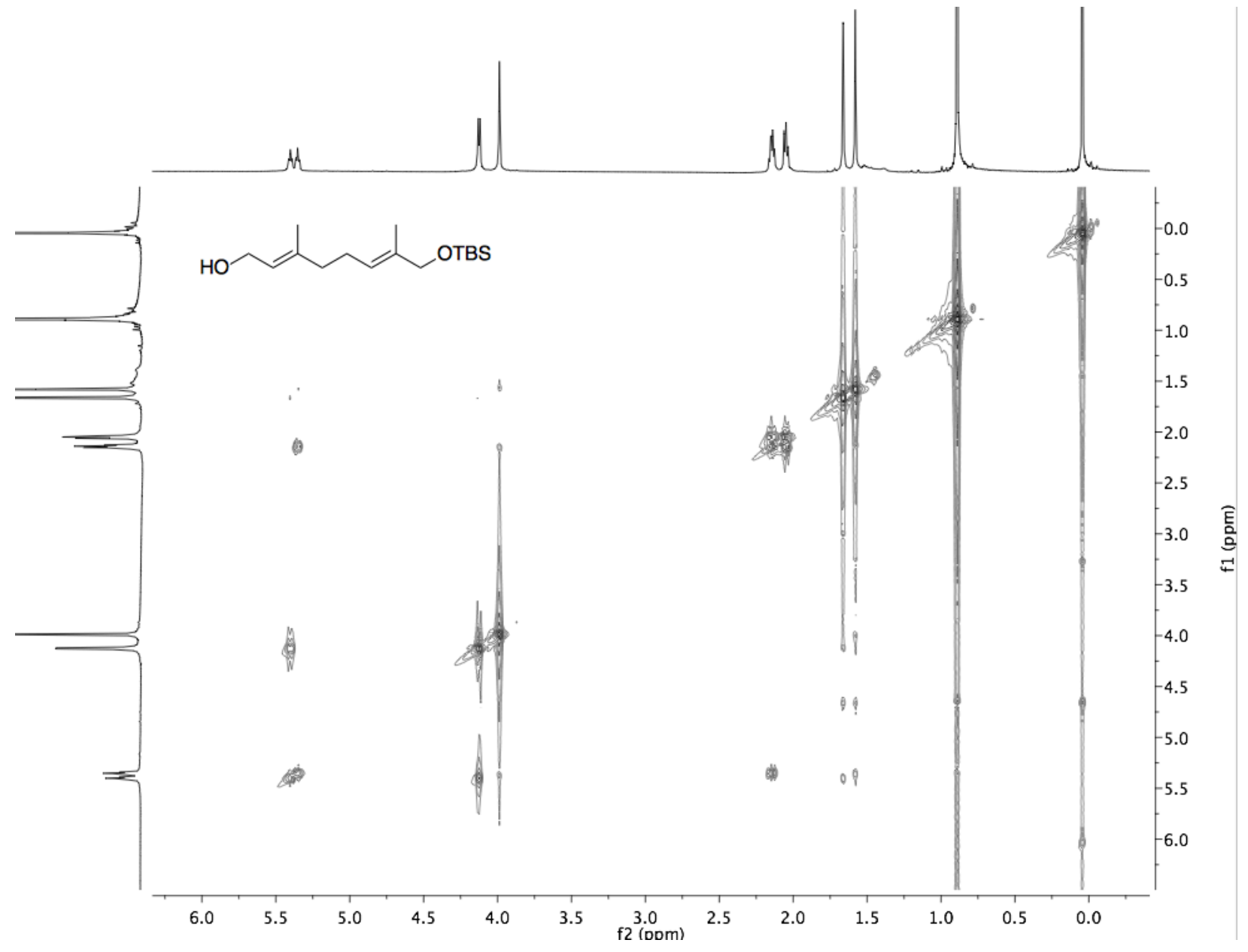




\section{${ }^{1} \mathrm{H}$ NMR Spectrum of 4}

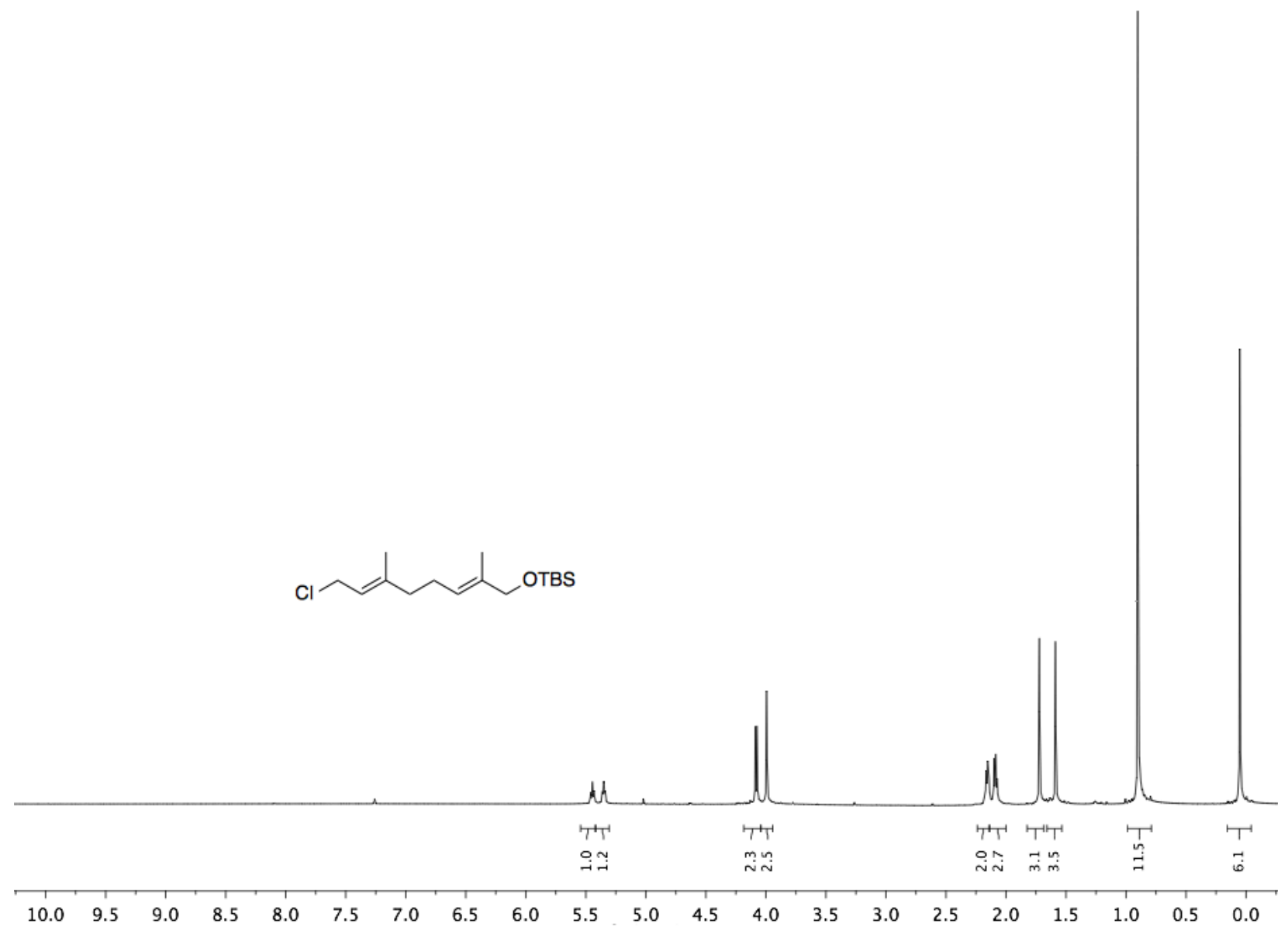

${ }^{13}$ C NMR Spectrum of 4

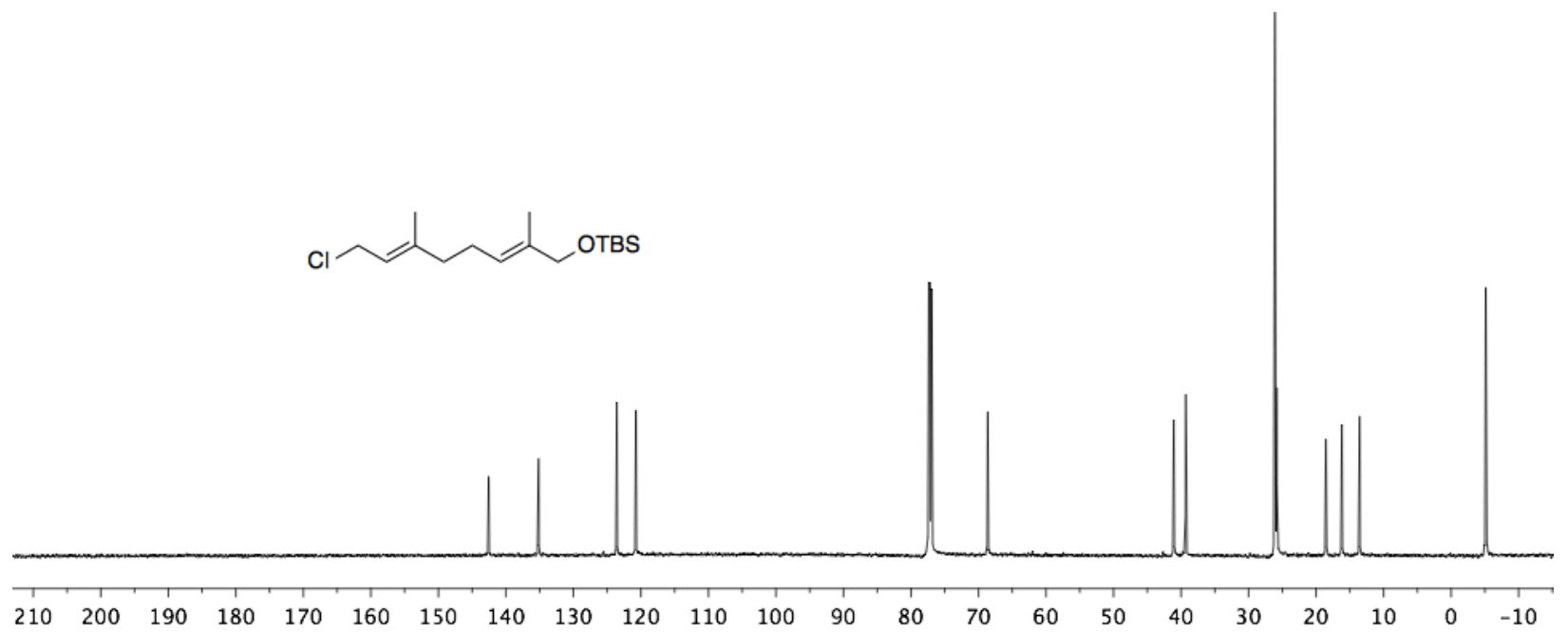




\section{${ }^{1} \mathrm{H}-{ }^{1} \mathrm{H}$ COSY NMR Spectrum of 4}

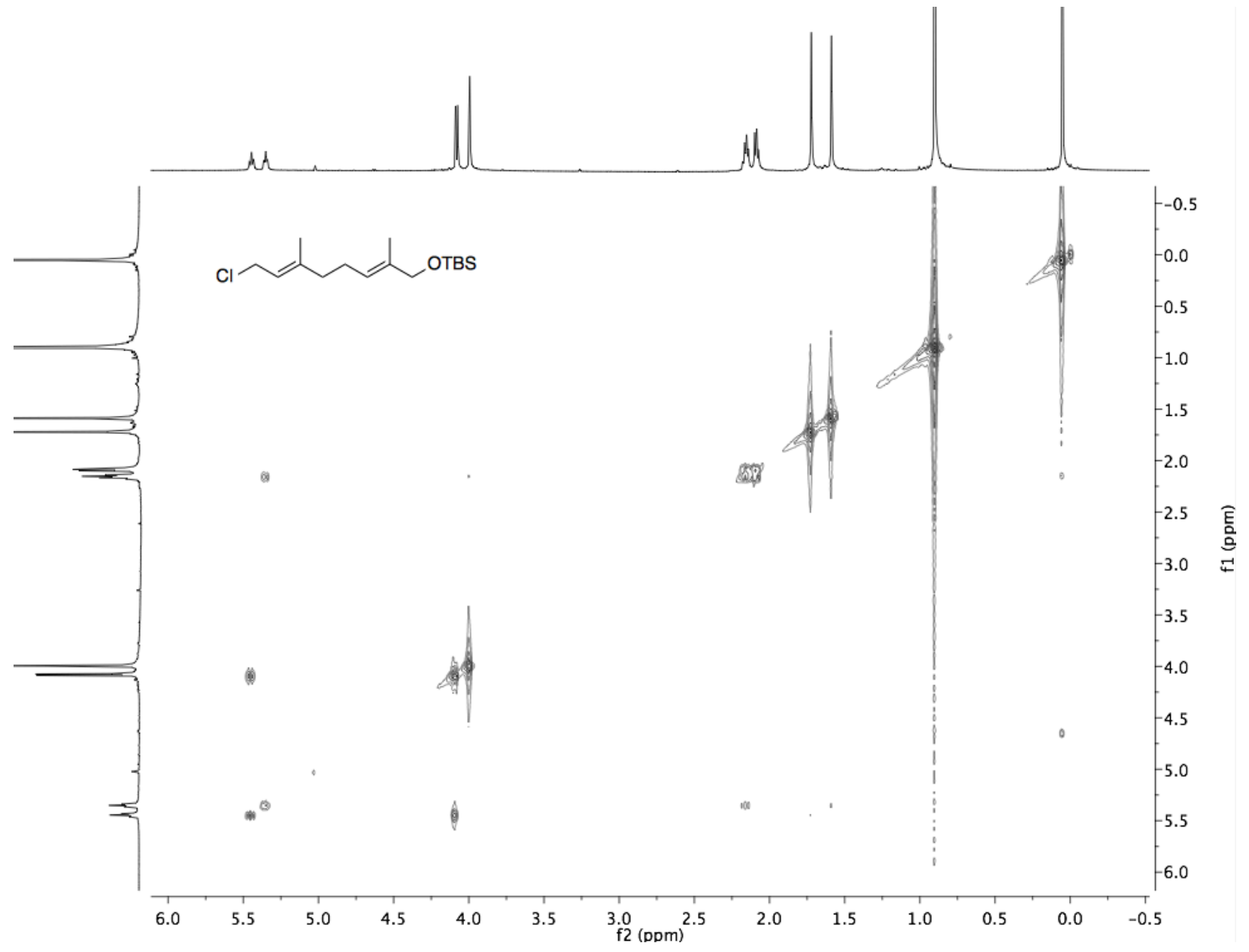


${ }^{1}$ H NMR Spectrum of 5A (partially purified)

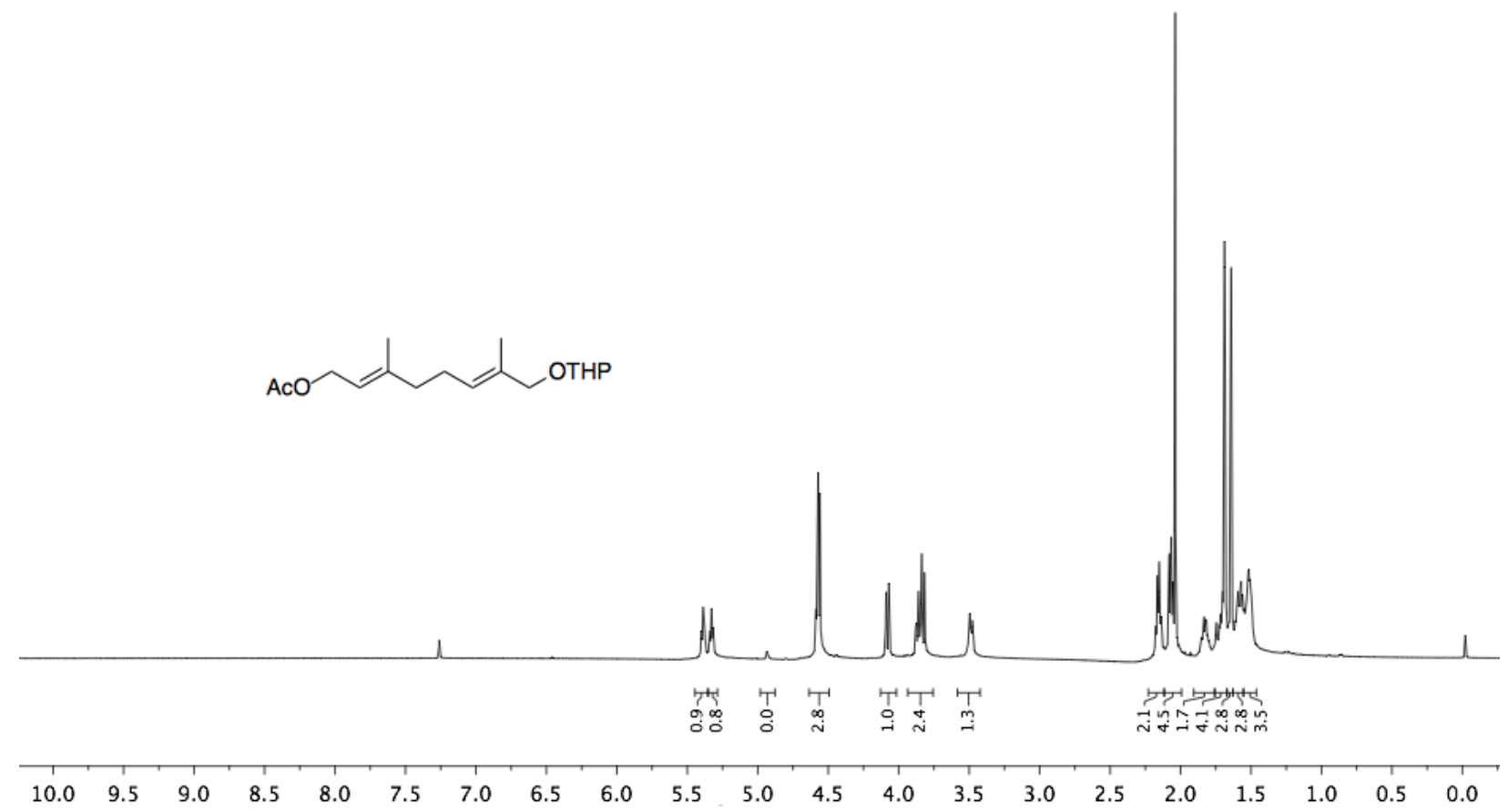

${ }^{13}$ C NMR Spectrum of $5 \mathrm{~A}$ (partially purified)

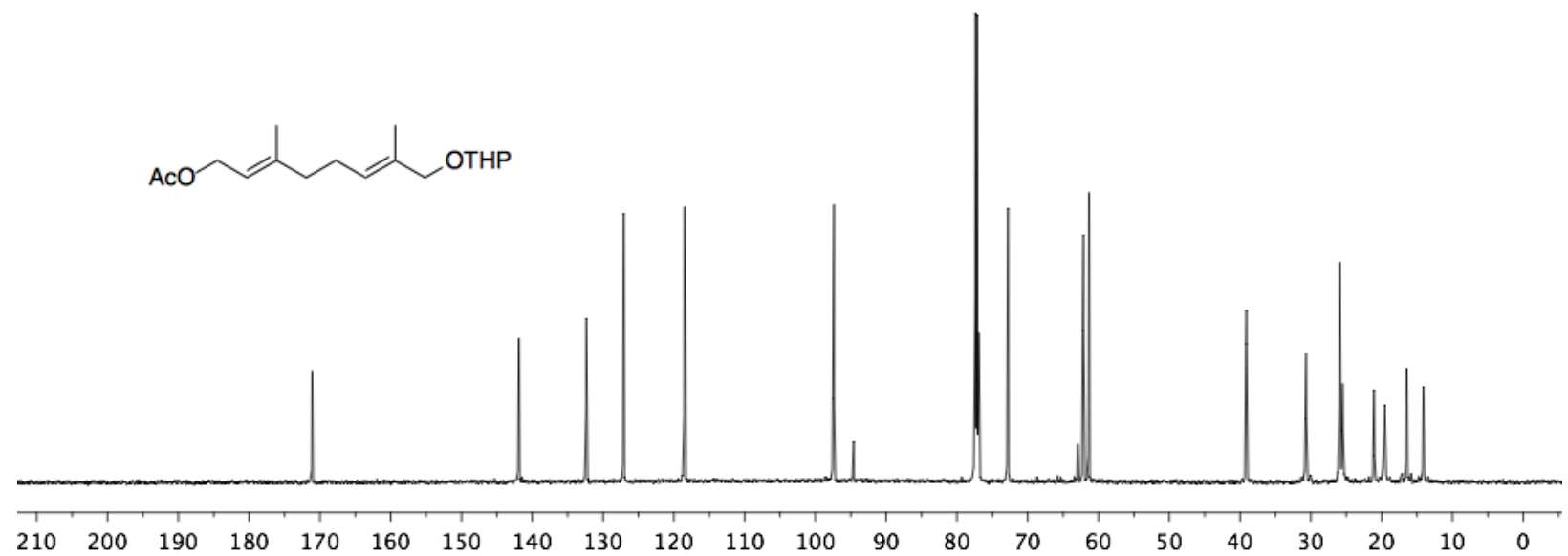


${ }^{1} \mathrm{H}-{ }^{1} \mathrm{H}$ COSY NMR Spectrum of 5A (partially purified)

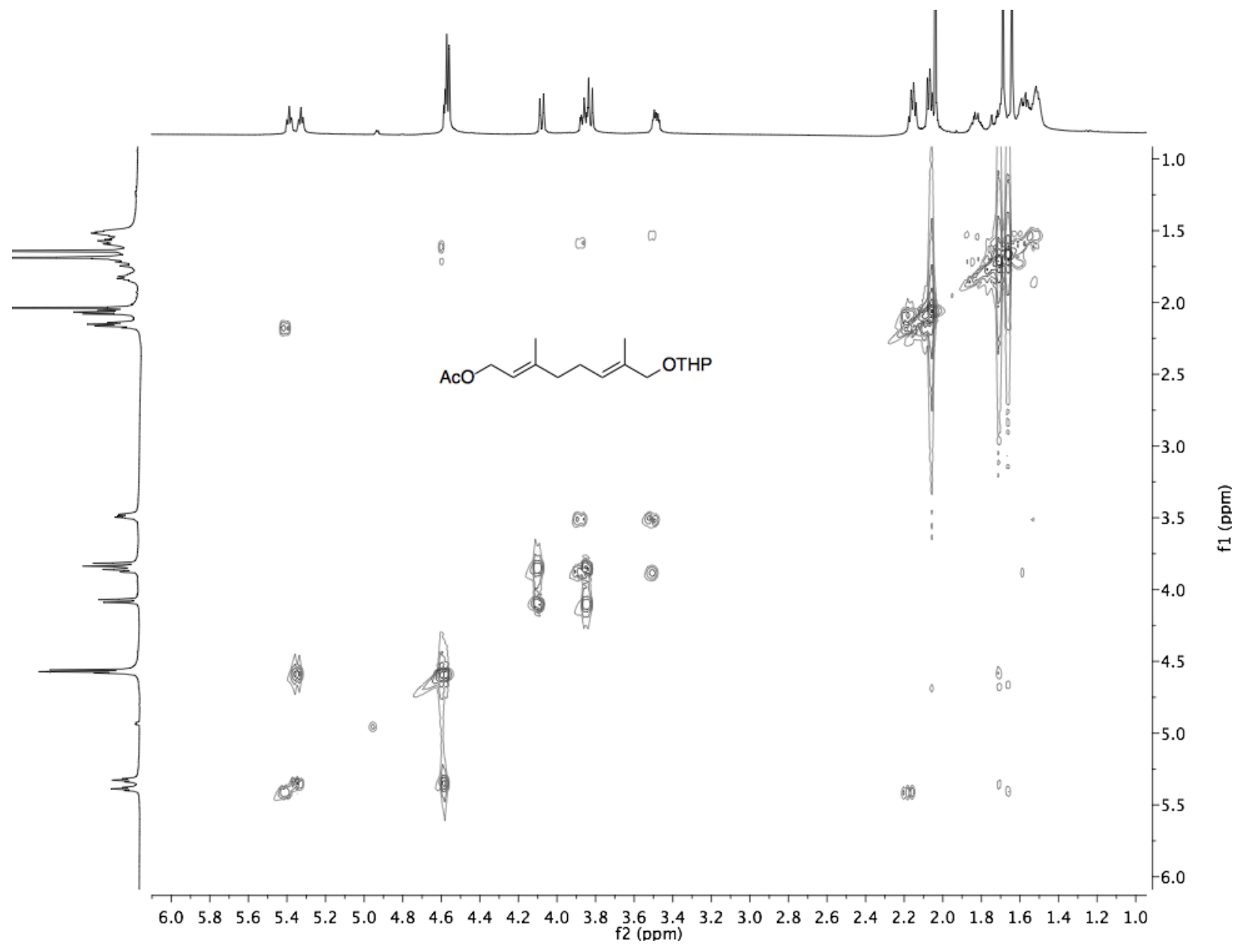

${ }^{1}$ H NMR Spectrum of 5B

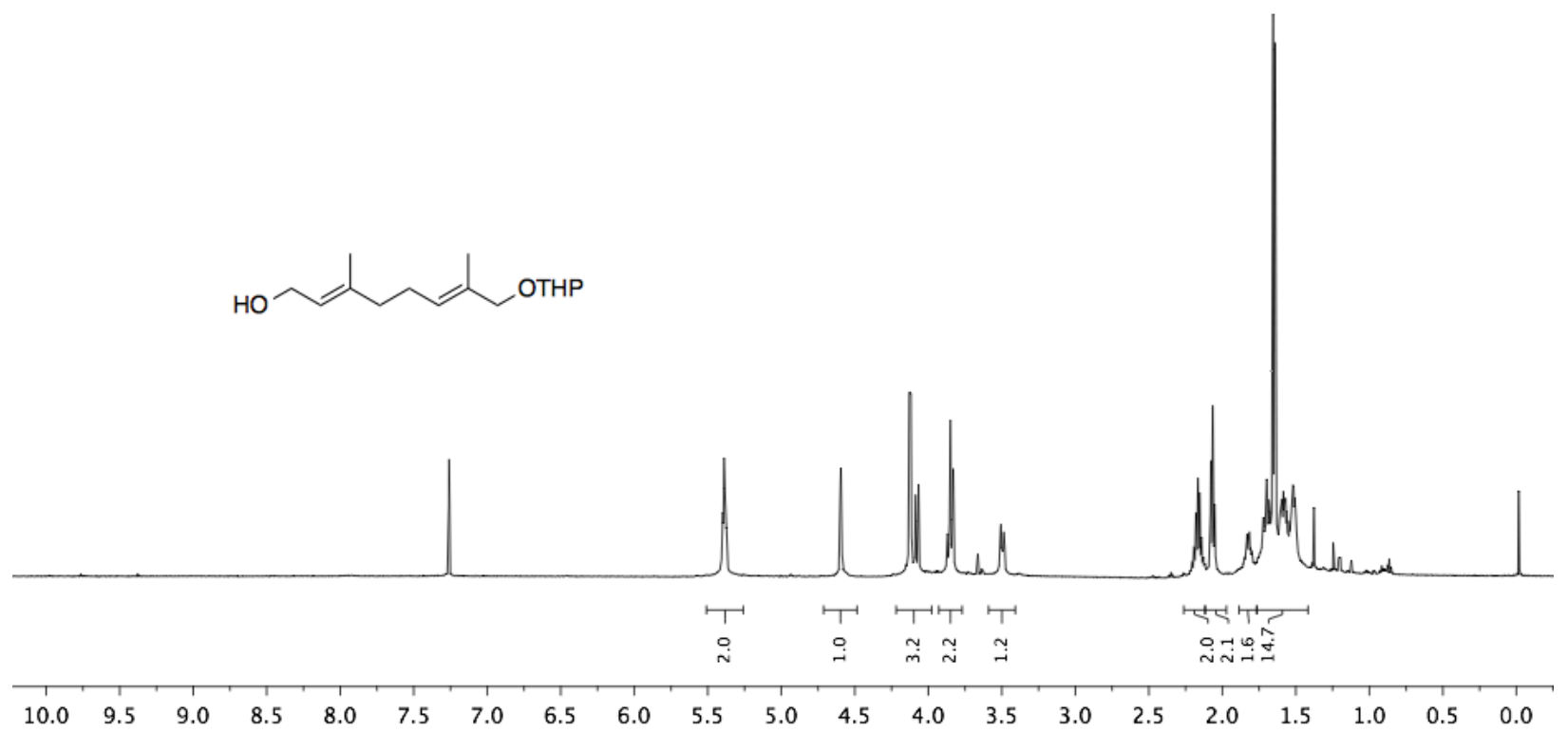


${ }^{13} \mathrm{C}$ NMR Spectrum of 5B

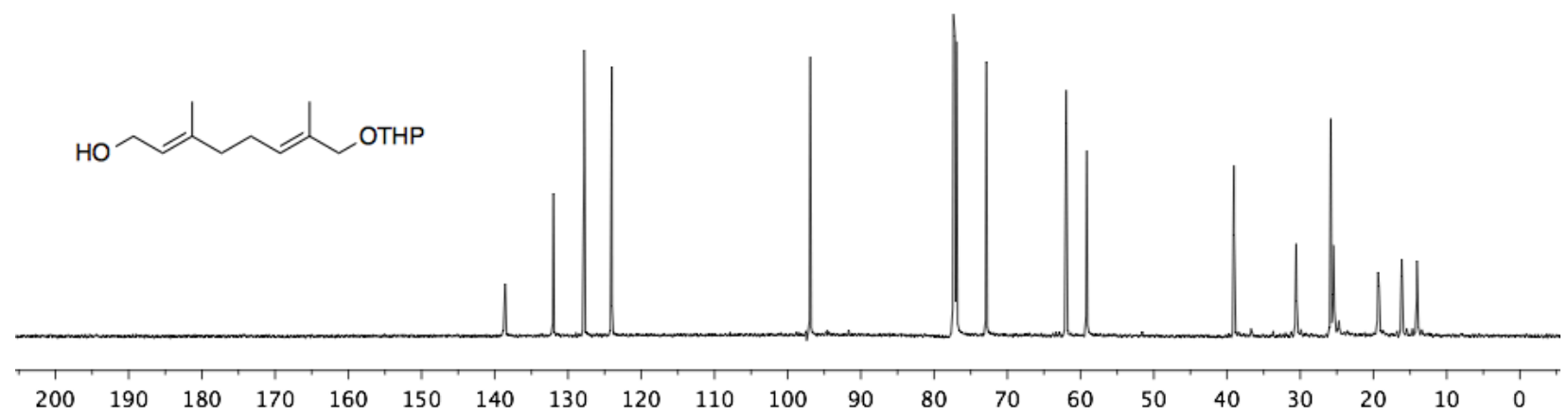

${ }^{1} \mathrm{H}-{ }^{1} \mathrm{H}$ COSY NMR Spectrum of $5 B$

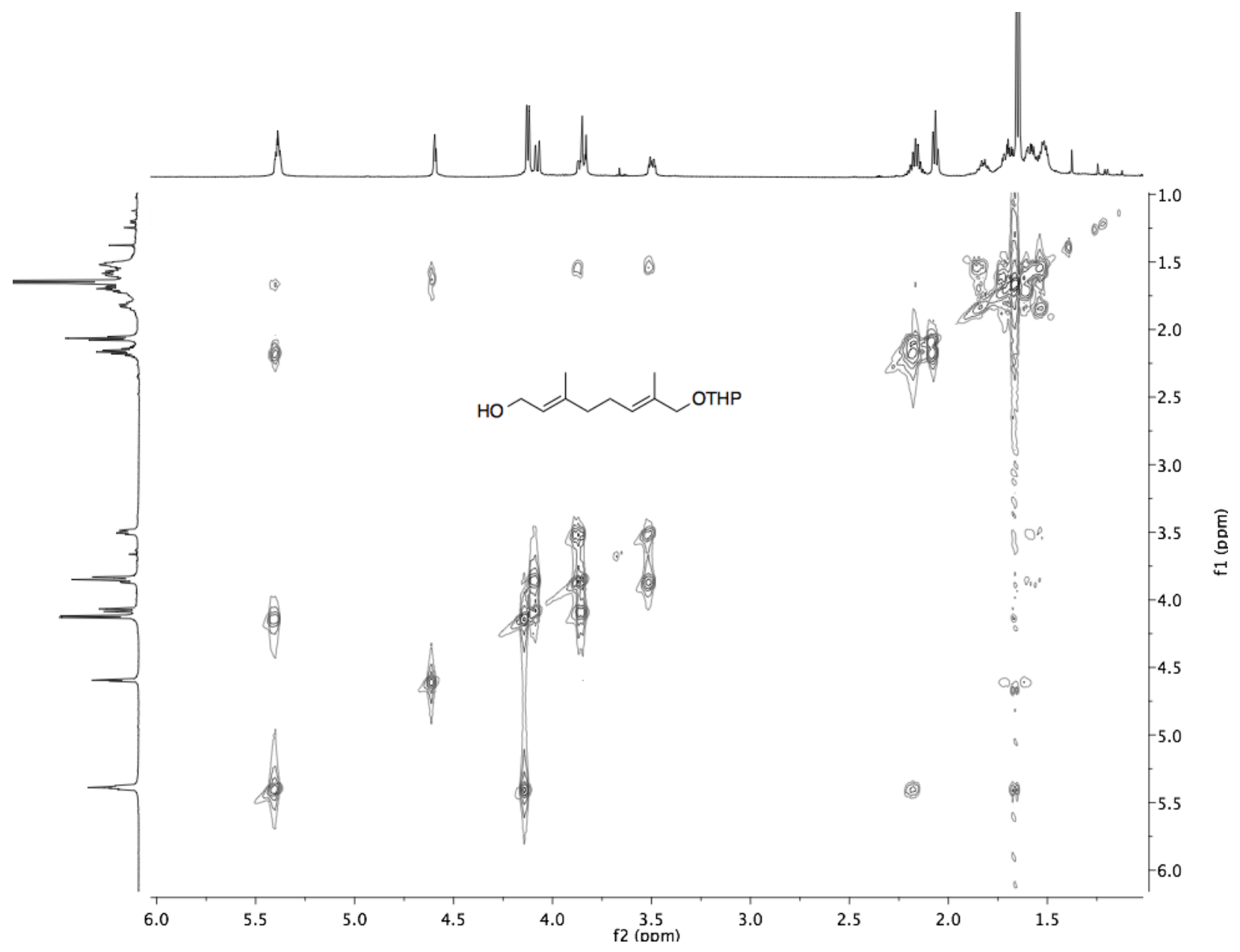




\section{${ }^{1} \mathrm{H}$ NMR Spectrum of 5}

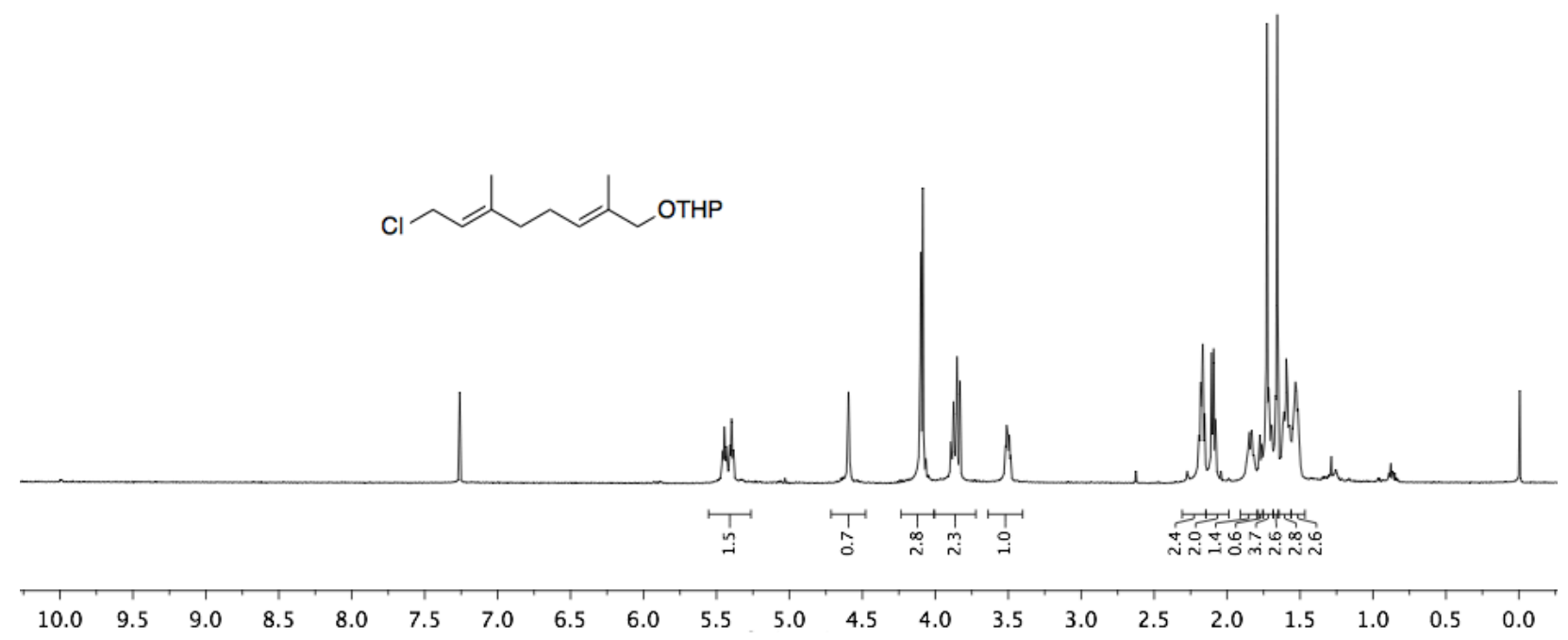

${ }^{13} \mathrm{C}$ NMR Spectrum of 5

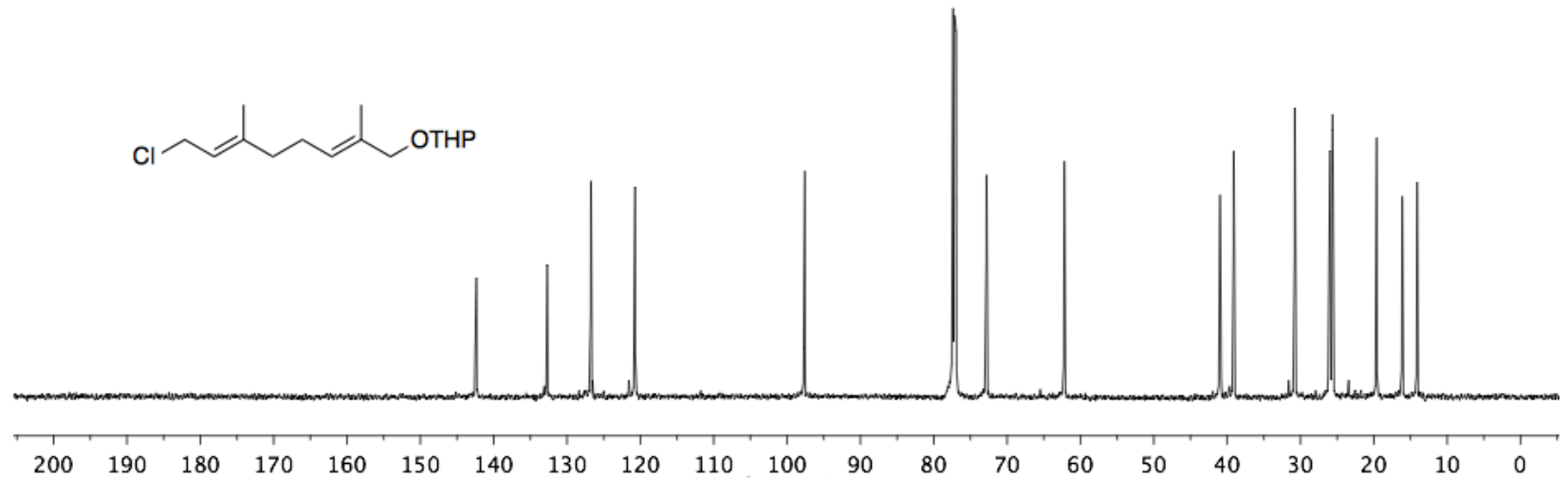




\section{${ }^{1} \mathrm{H}-{ }^{1} \mathrm{H}$ COSY NMR Spectrum of 5}

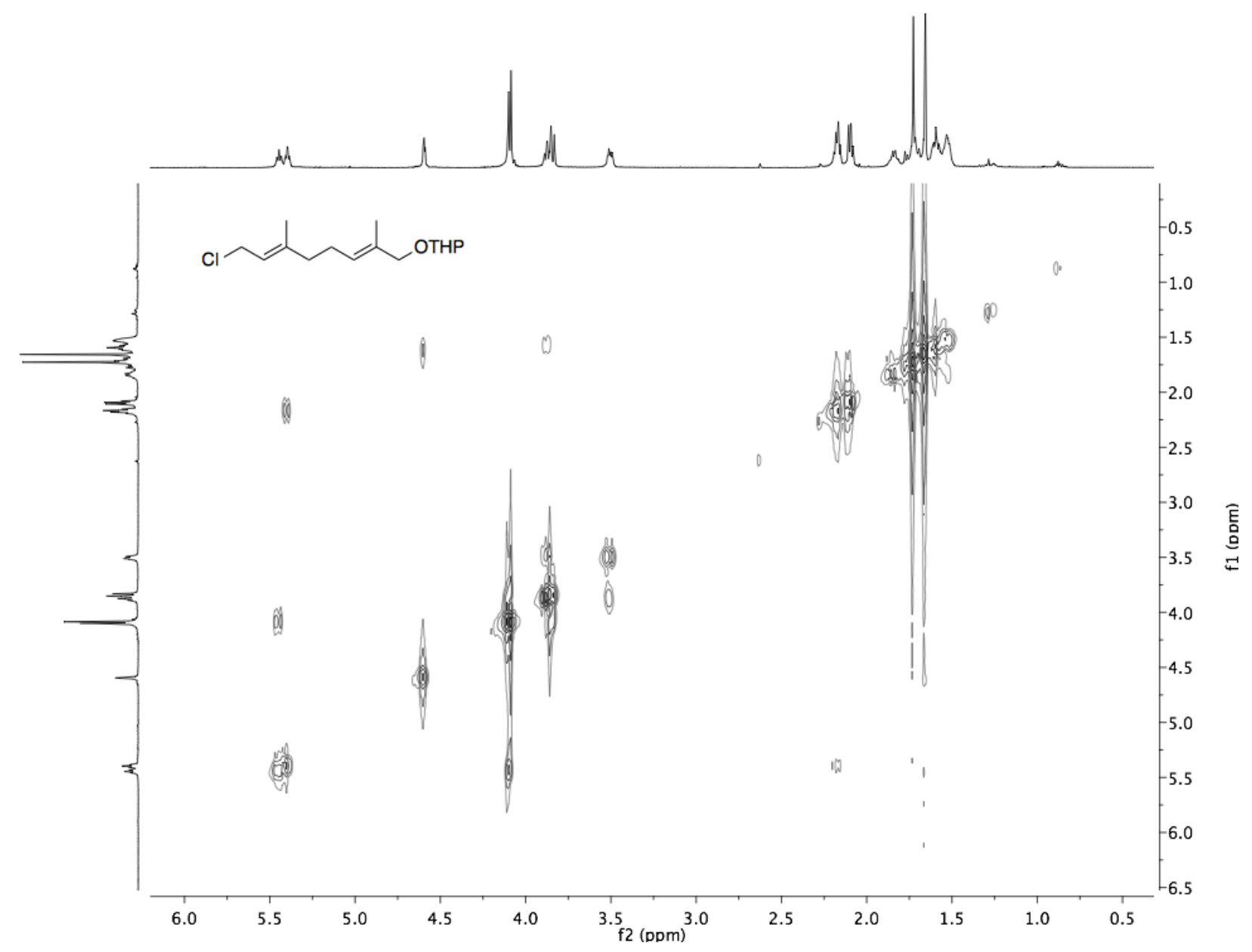




\section{${ }^{1}$ H NMR Spectrum of 7}

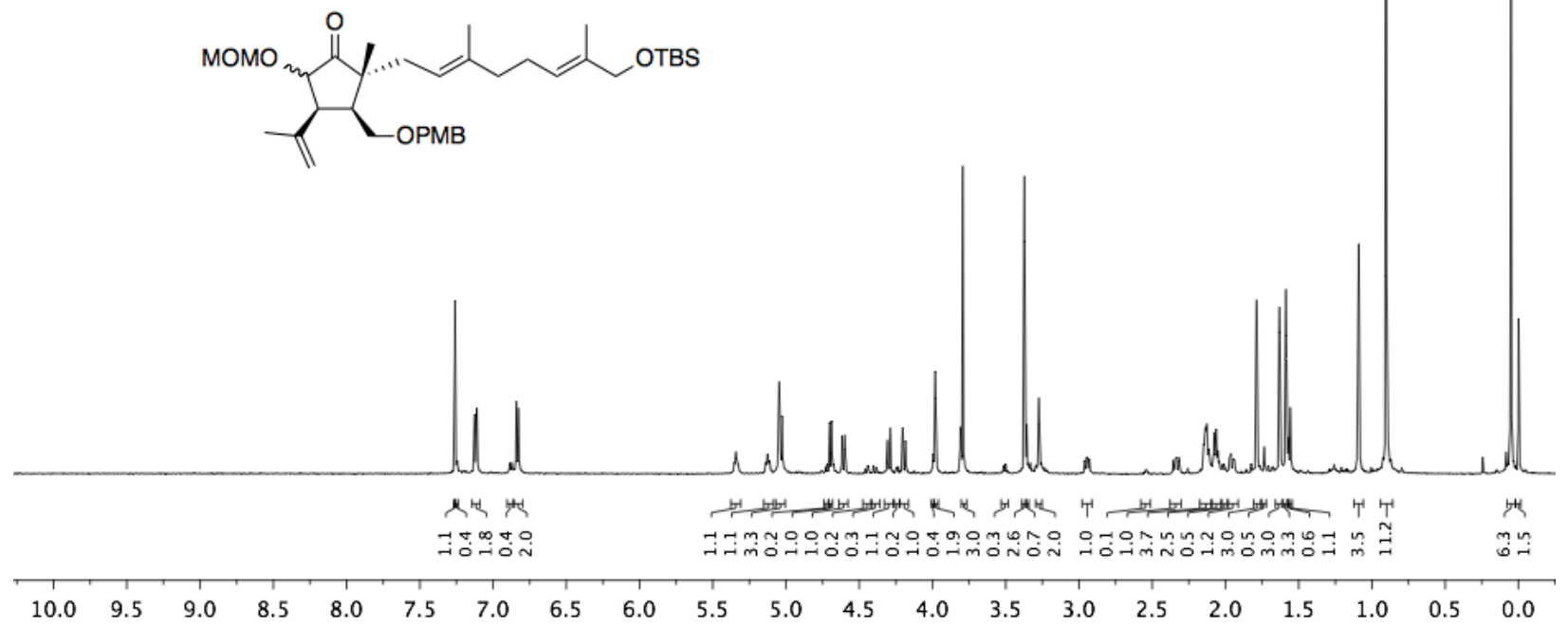


${ }^{13} \mathrm{C}$ NMR Spectrum of 7

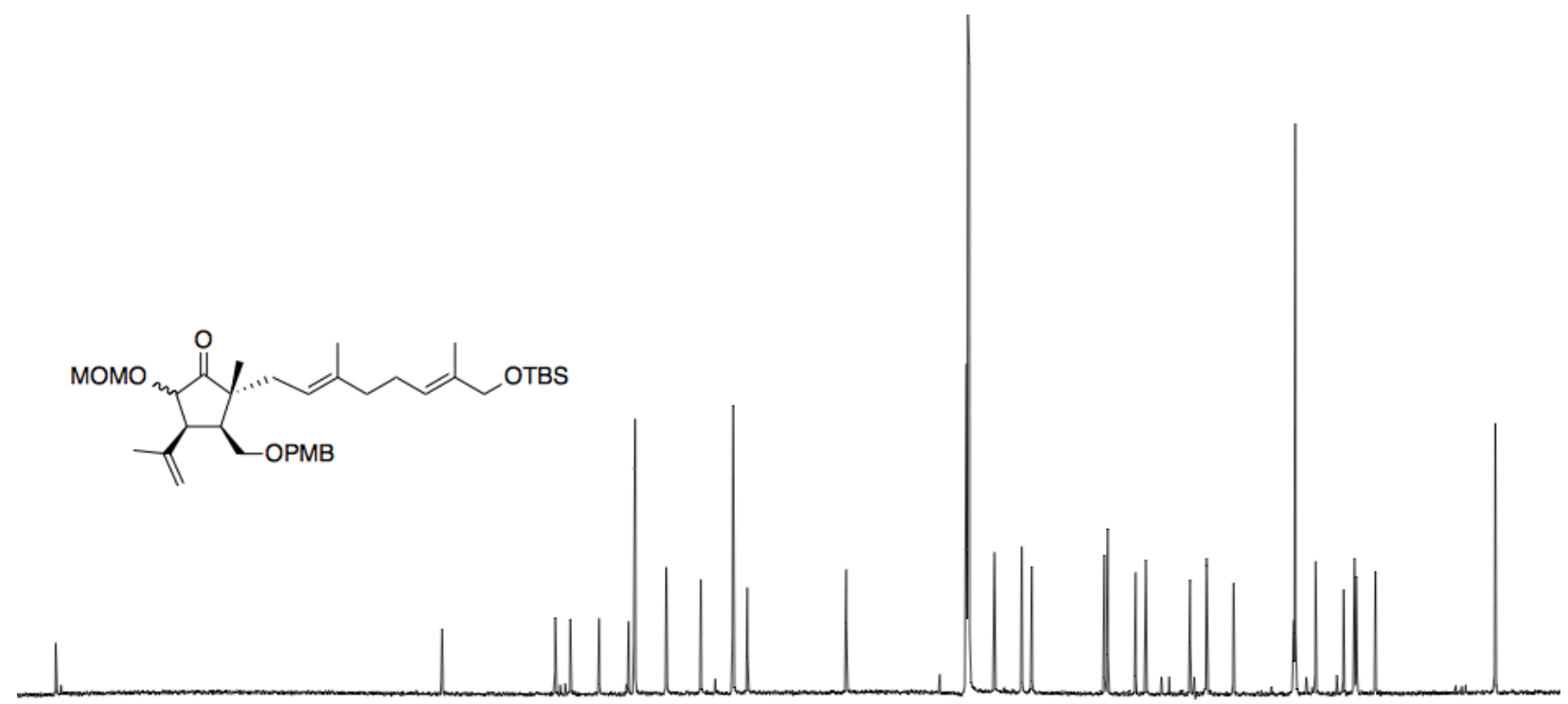

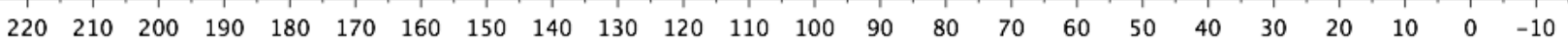




\section{${ }^{1} \mathrm{H}-{ }^{1} \mathrm{H}$ COSY NMR Spectrum of 7}

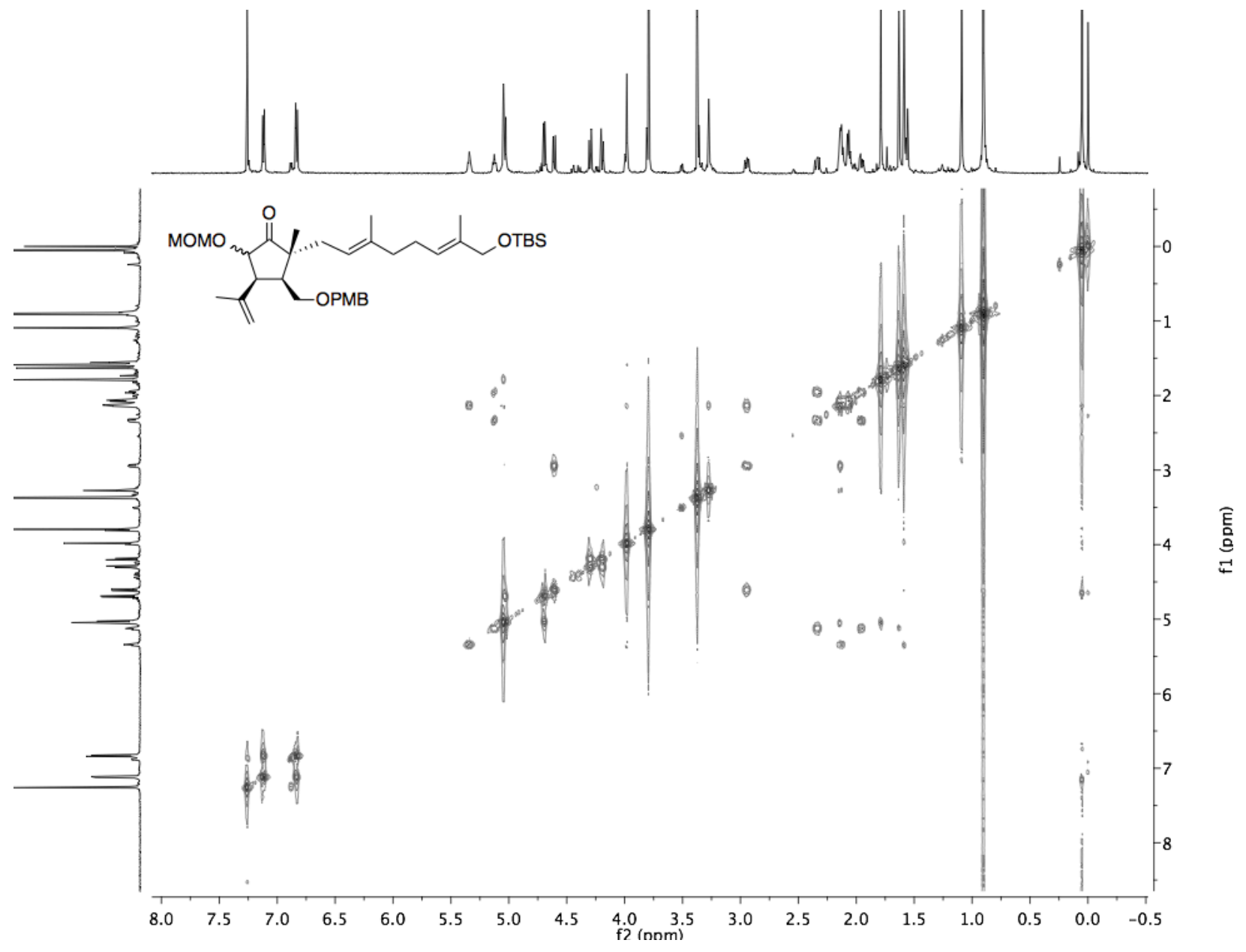




\section{${ }^{1} \mathrm{H}-{ }^{1} \mathrm{H}$ NOESY NMR Spectrum of 7}

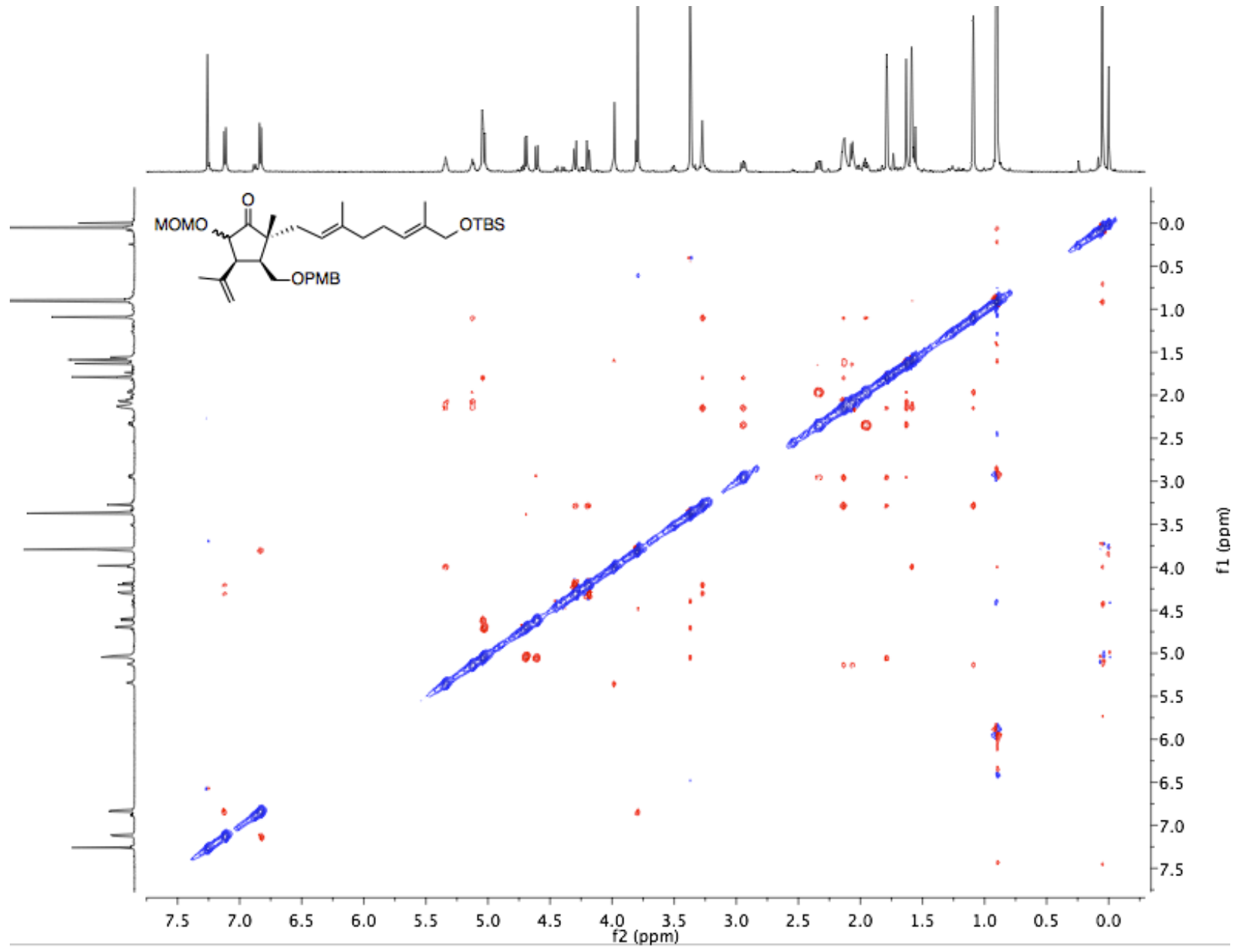




\section{${ }^{1}$ H NMR Spectrum of 8}

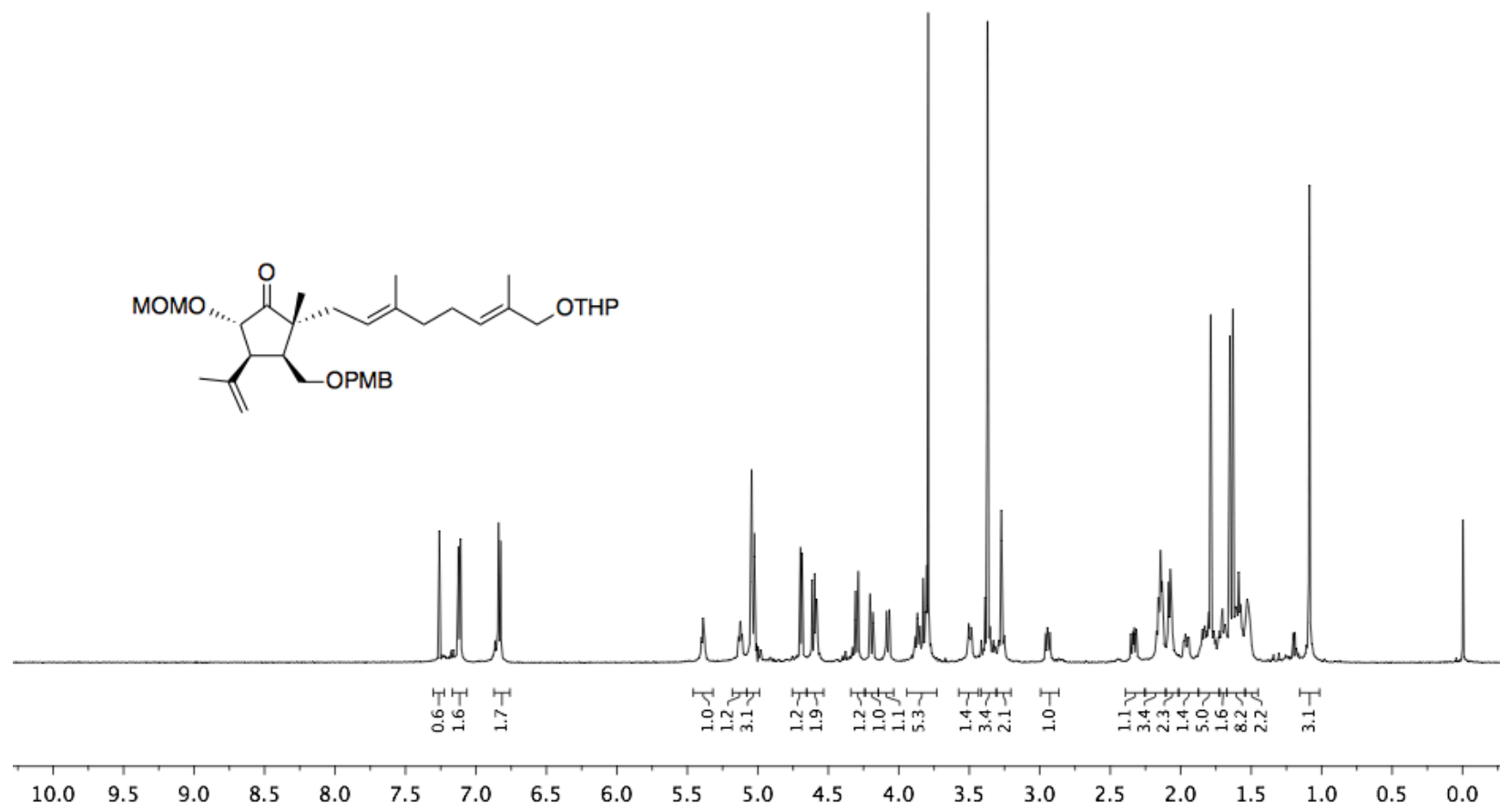

${ }^{13} \mathrm{C}$ NMR Spectrum of 8

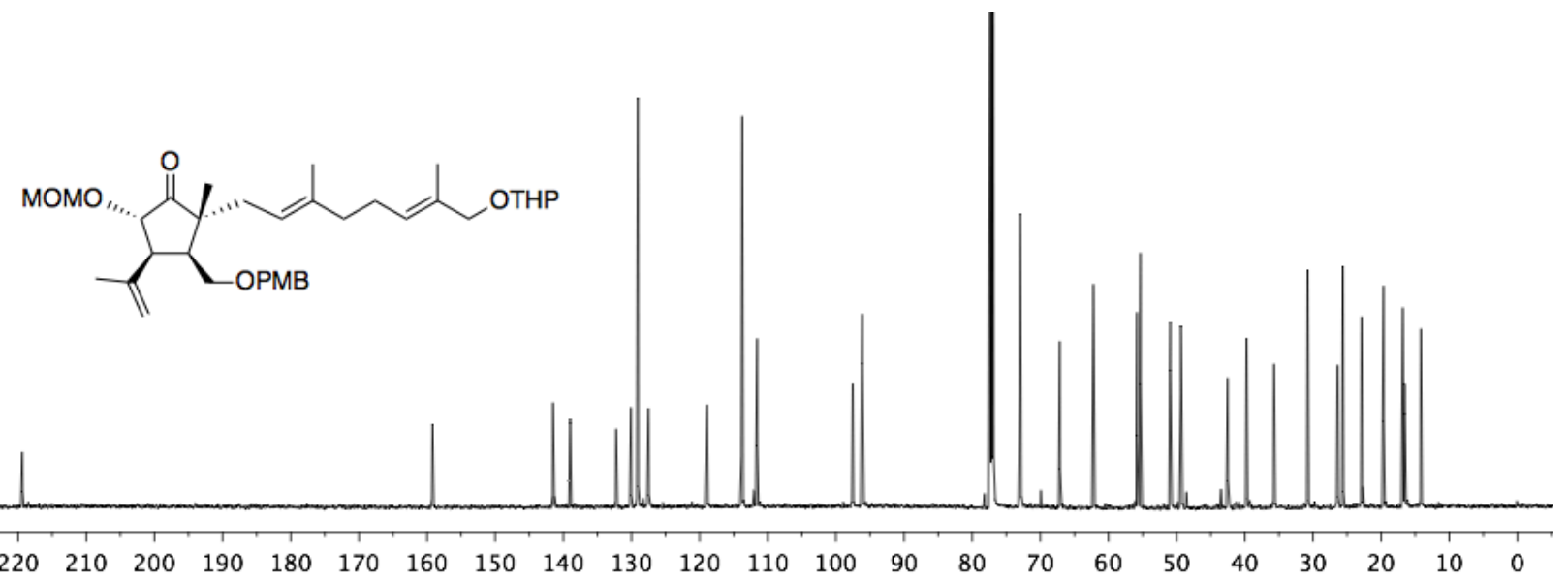




\section{${ }^{1} \mathrm{H}-{ }^{1} \mathrm{H}$ COSY NMR Spectrum of 8}

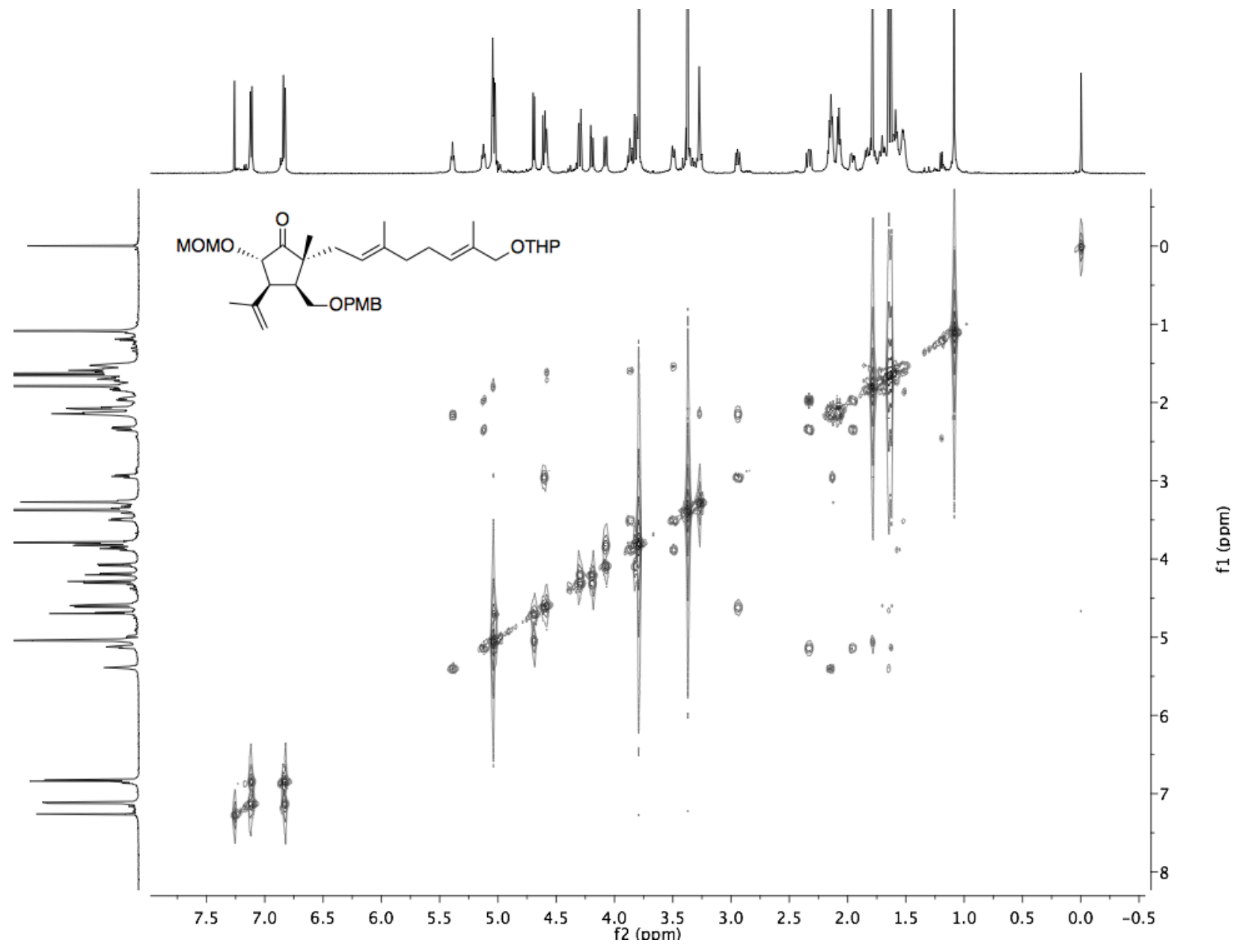


${ }^{1} \mathrm{H}-{ }^{1} \mathrm{H}$ NOSEY NMR Spectrum of 8

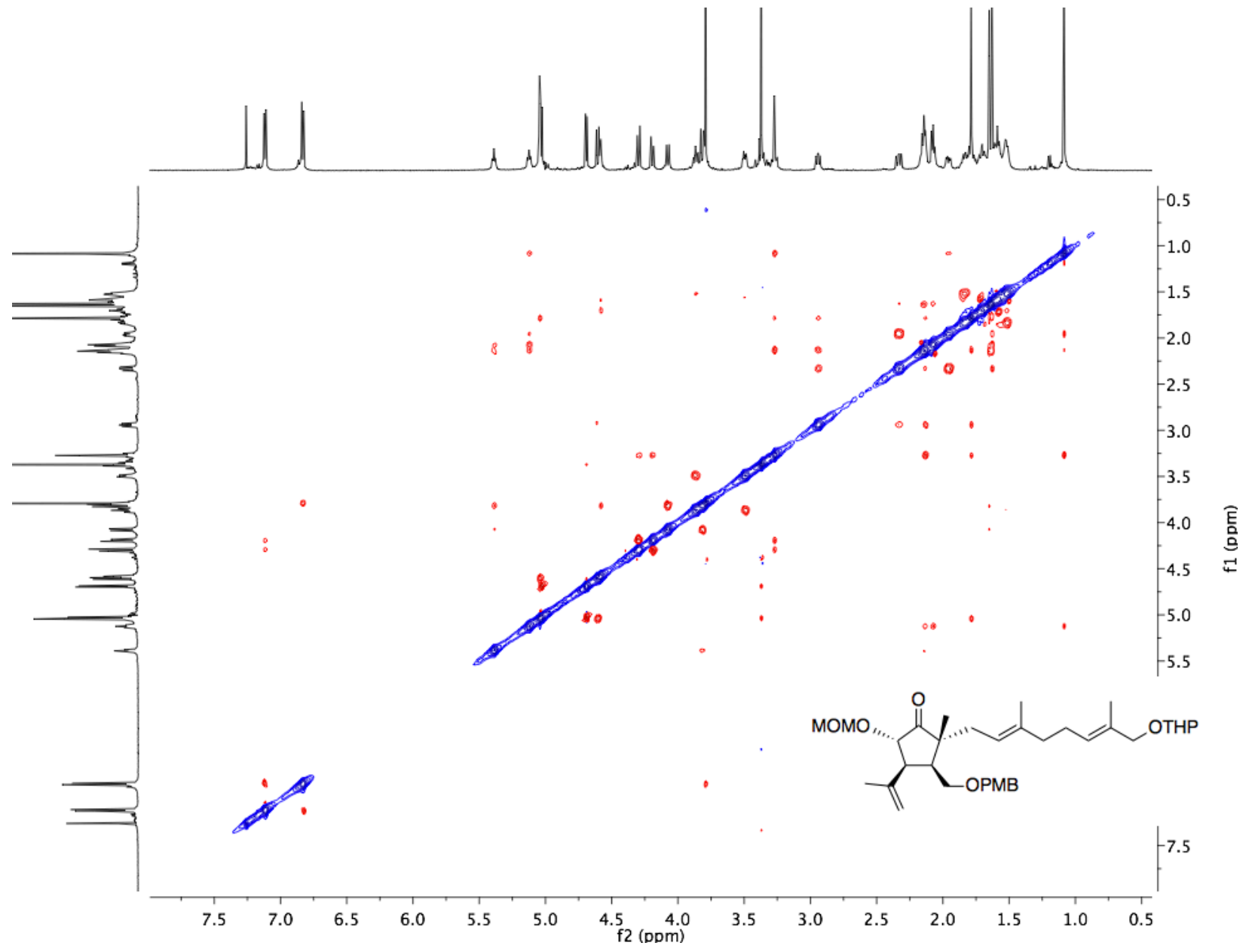

${ }^{1}$ H NMR Spectrum of 9

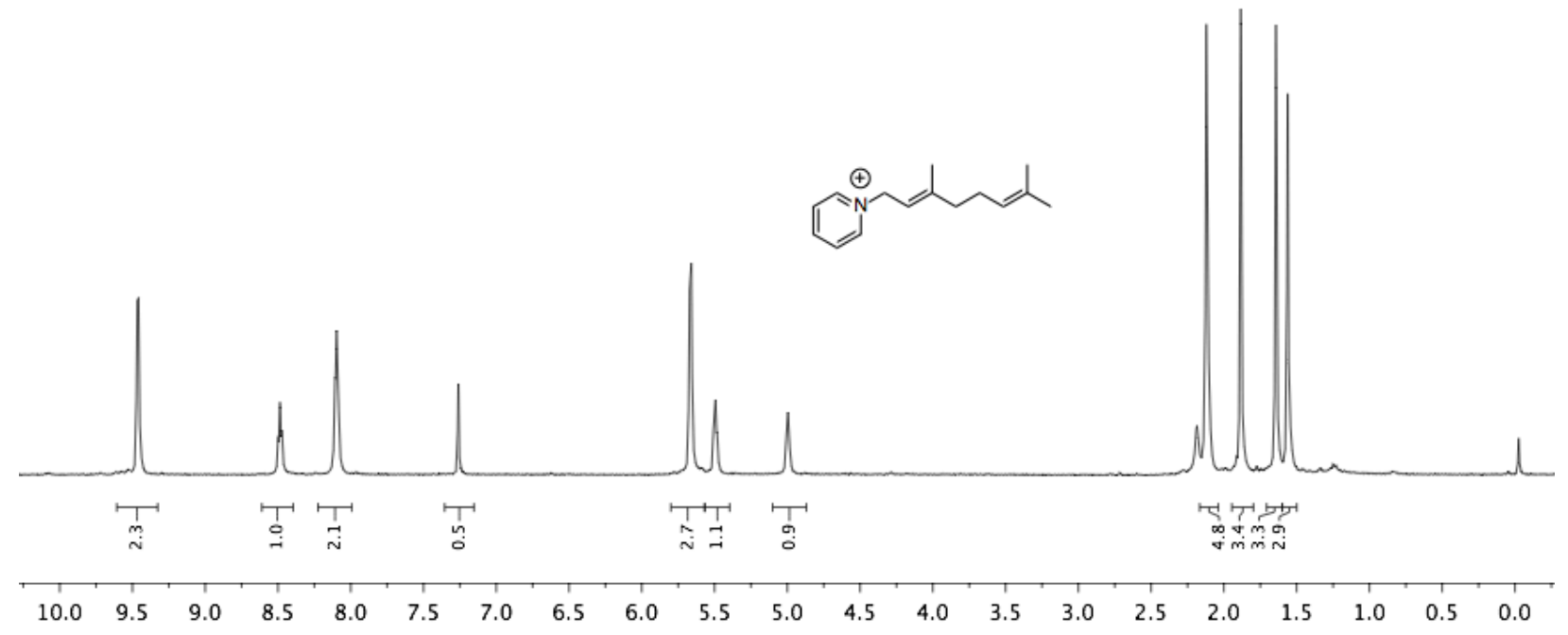


${ }^{13}$ C NMR Spectrum of 9

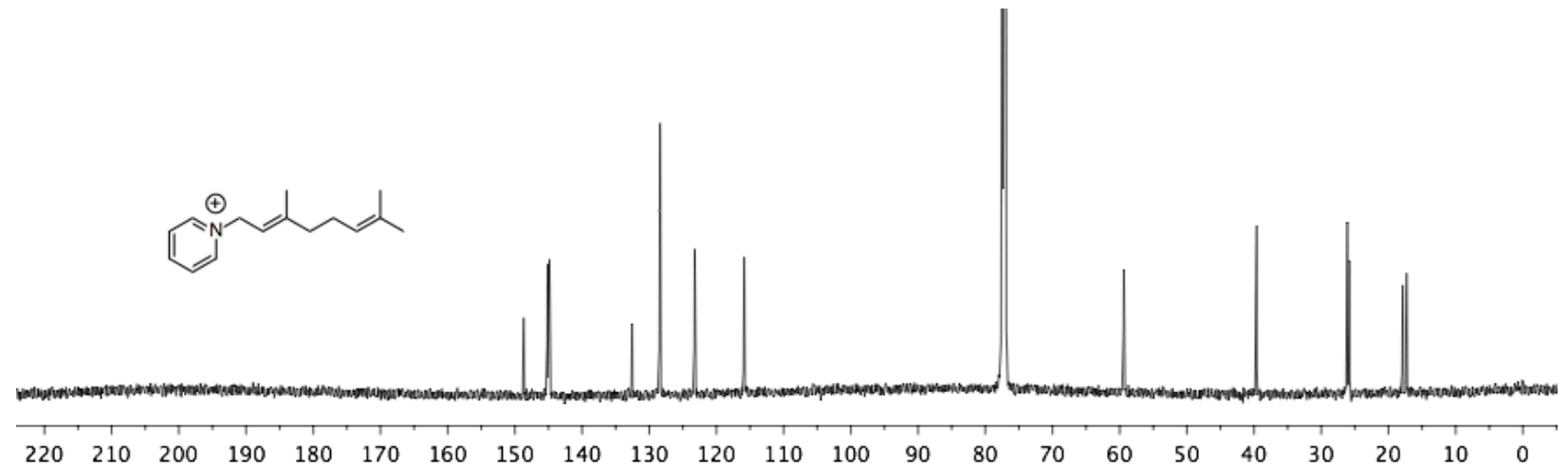

${ }^{1} \mathrm{H}-{ }^{1} \mathrm{H}$ COSY NMR Spectrum of 9

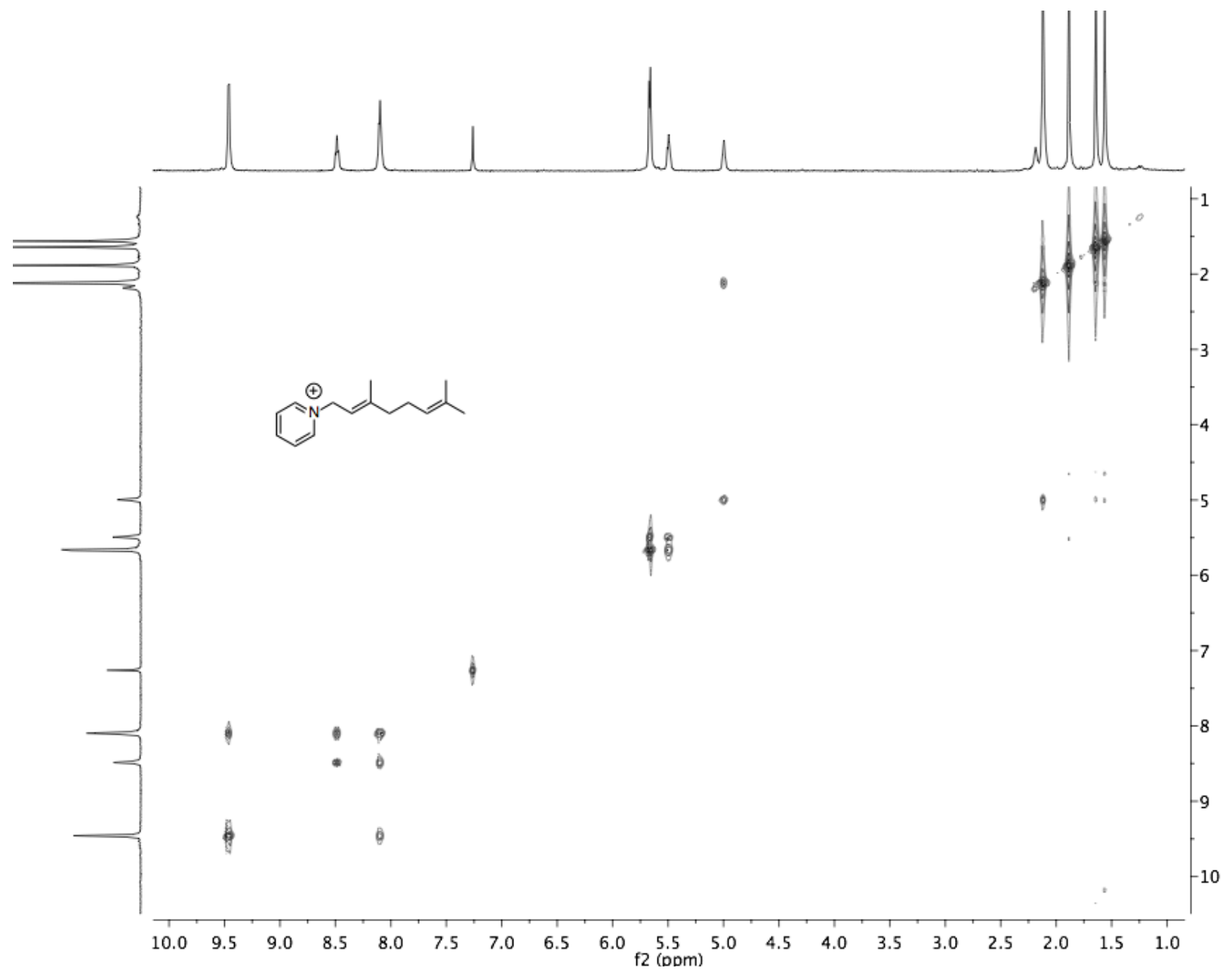




\section{${ }^{1} \mathrm{H}$ NMR Spectrum of 12}

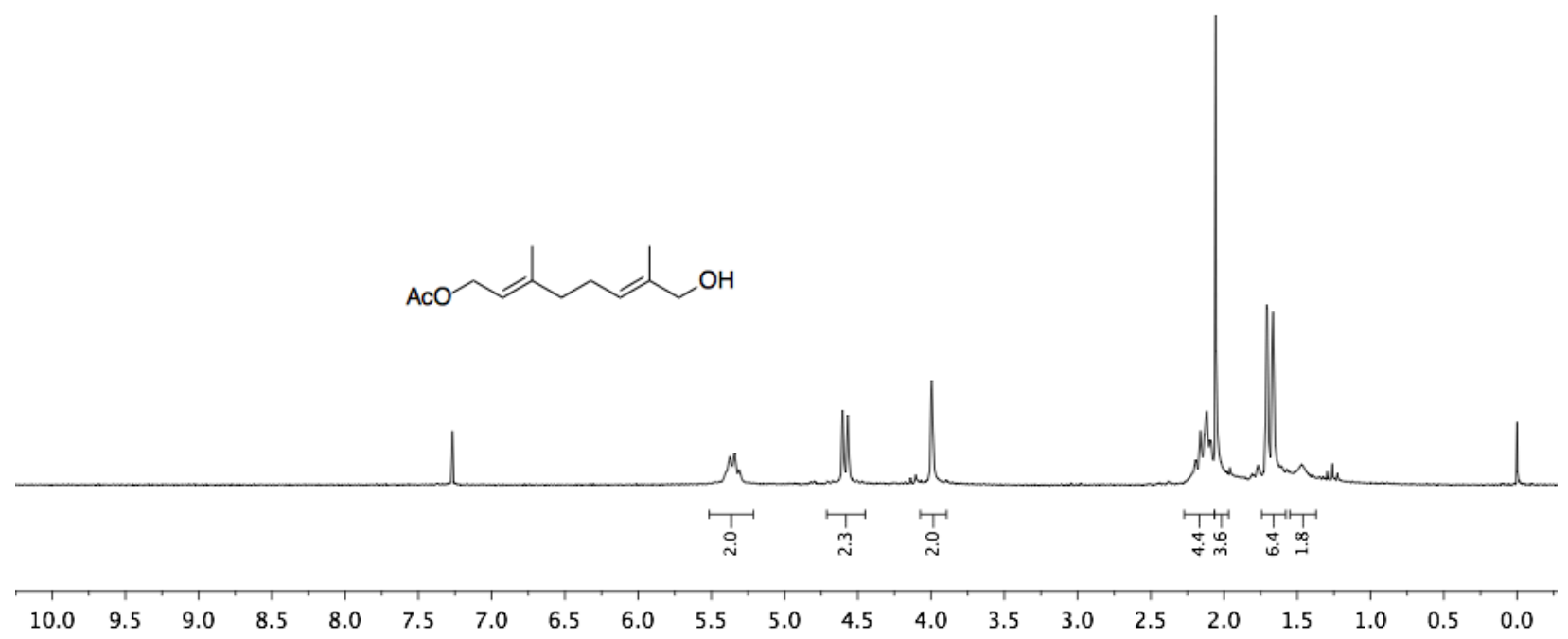

${ }^{1}$ H NMR Spectrum of 13 (crude)

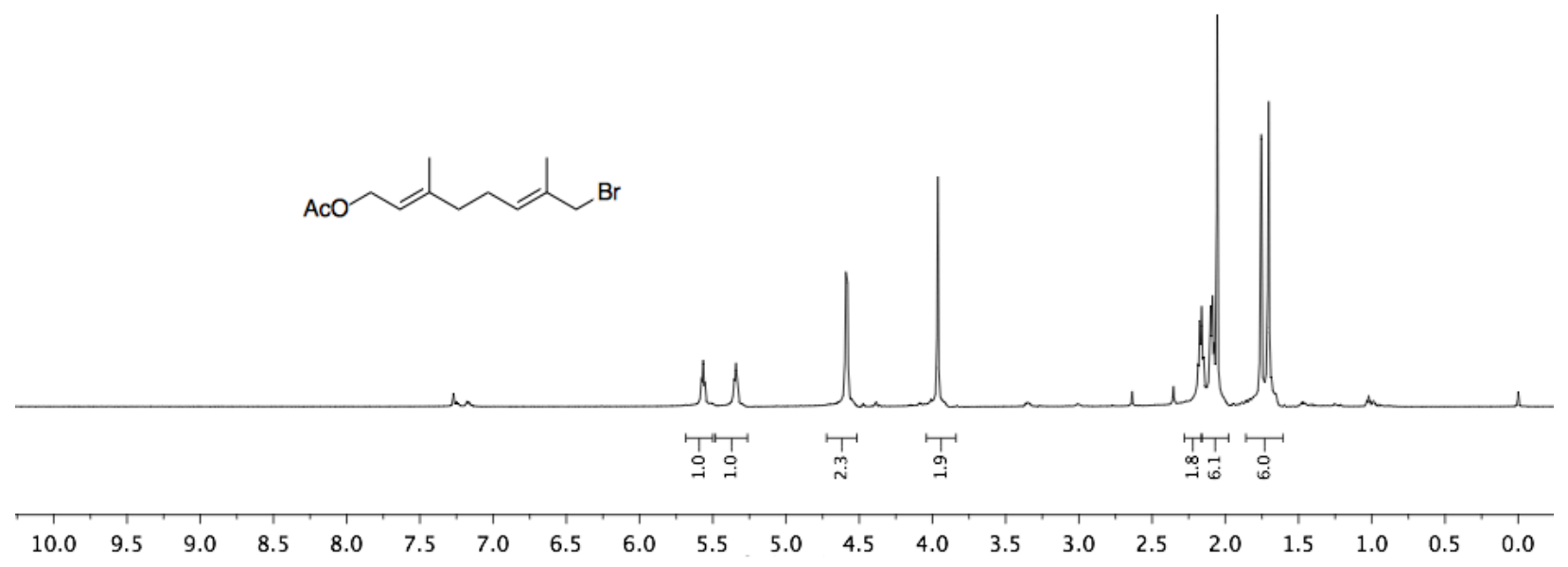

${ }^{13} \mathrm{C}$ NMR Spectrum of 13 (crude)

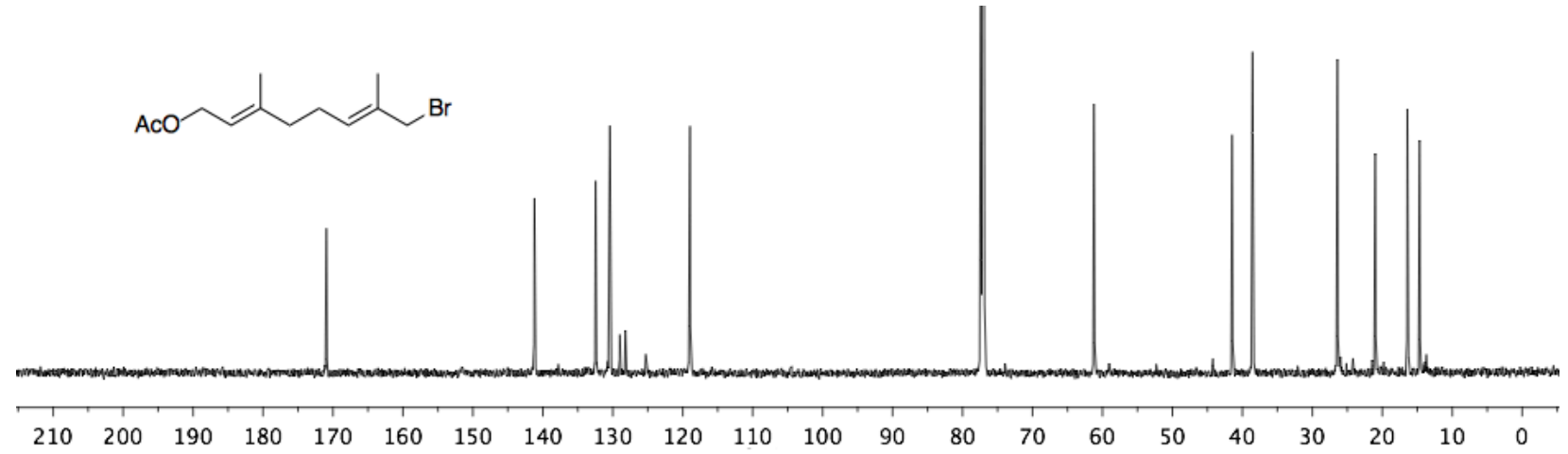




\section{${ }^{1} \mathrm{H}-{ }^{1} \mathrm{H}$ COSY NMR Spectrum of 13 (crude)}

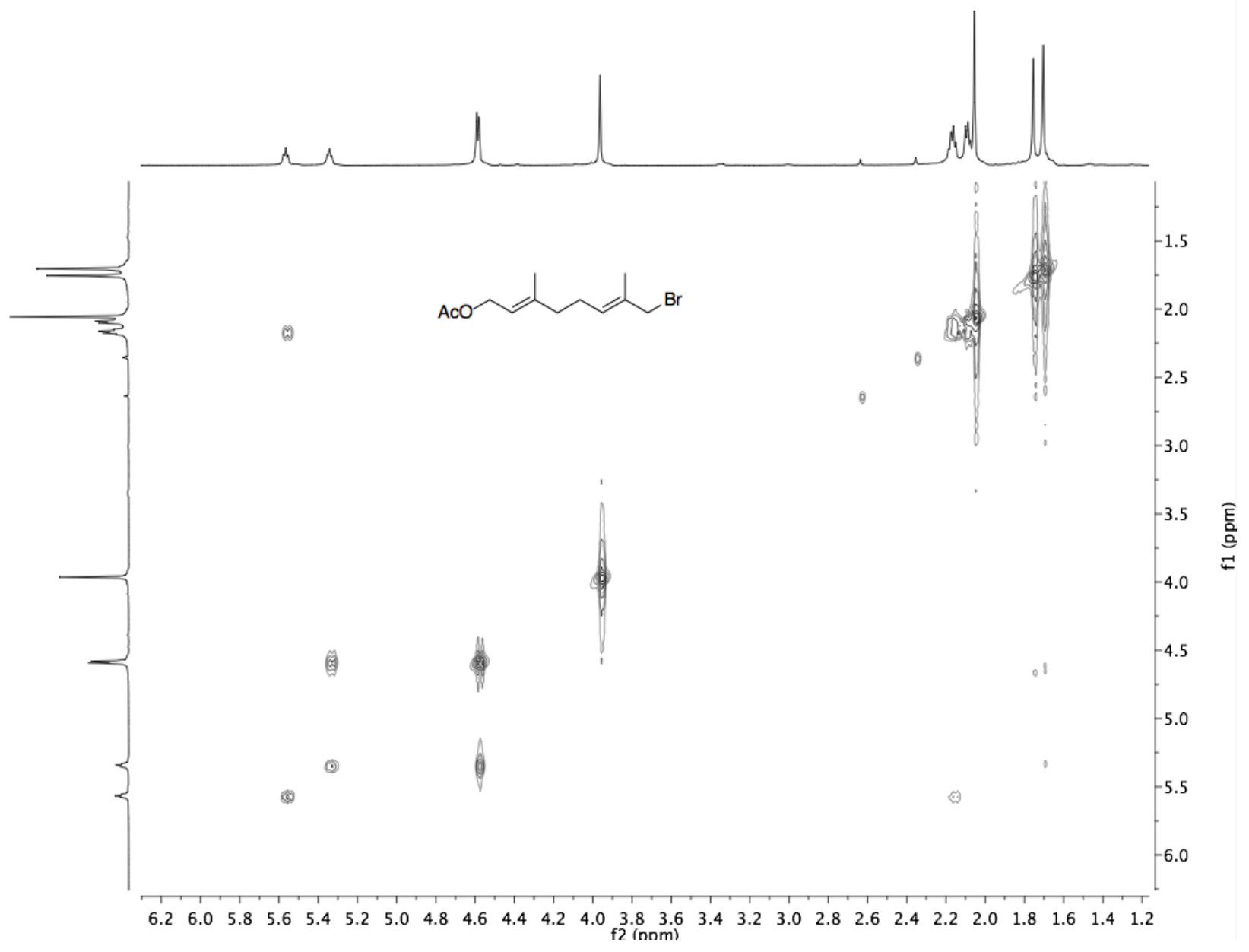

${ }^{1}$ H NMR Spectrum of 14

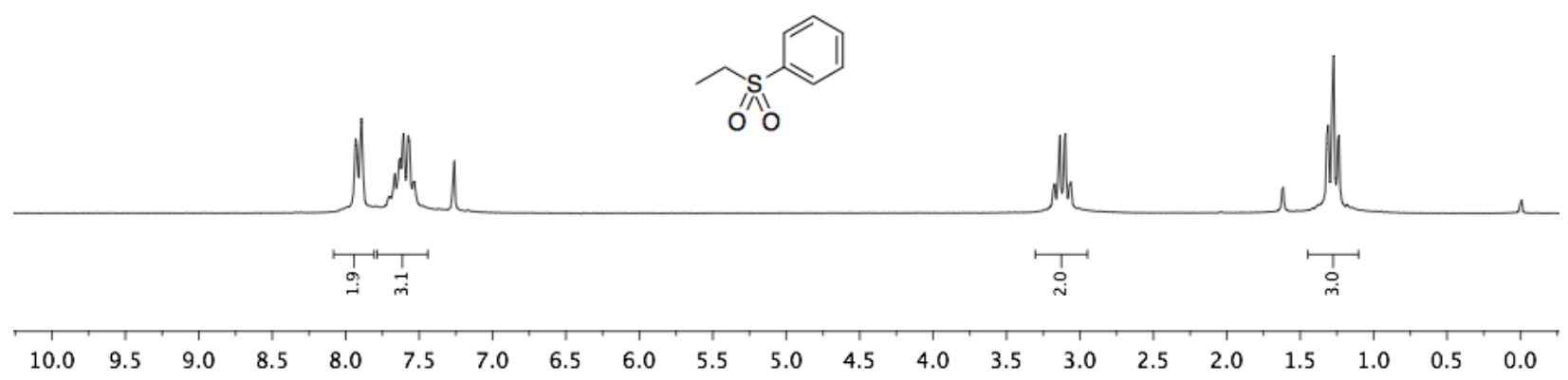




\section{${ }^{1} \mathrm{H}$ NMR Spectrum of 15}

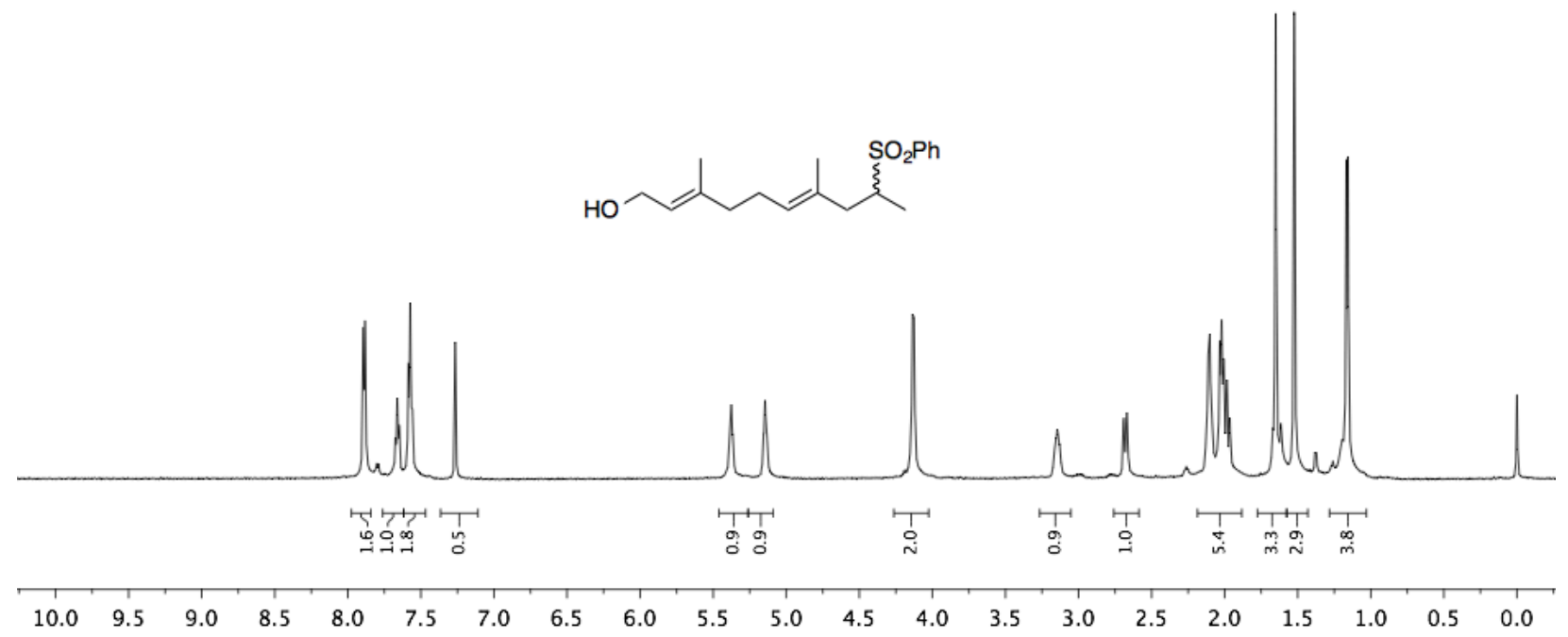

${ }^{13} \mathrm{C}$ NMR Spectrum of 15

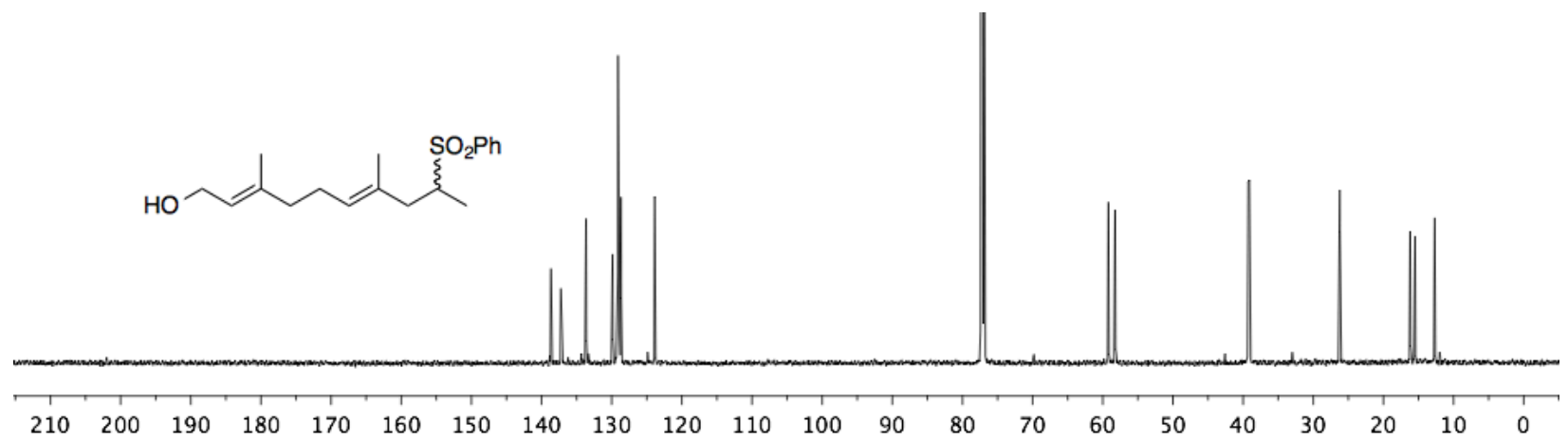




\section{${ }^{1} H-{ }^{1} H$ COSY NMR Spectrum of 15}

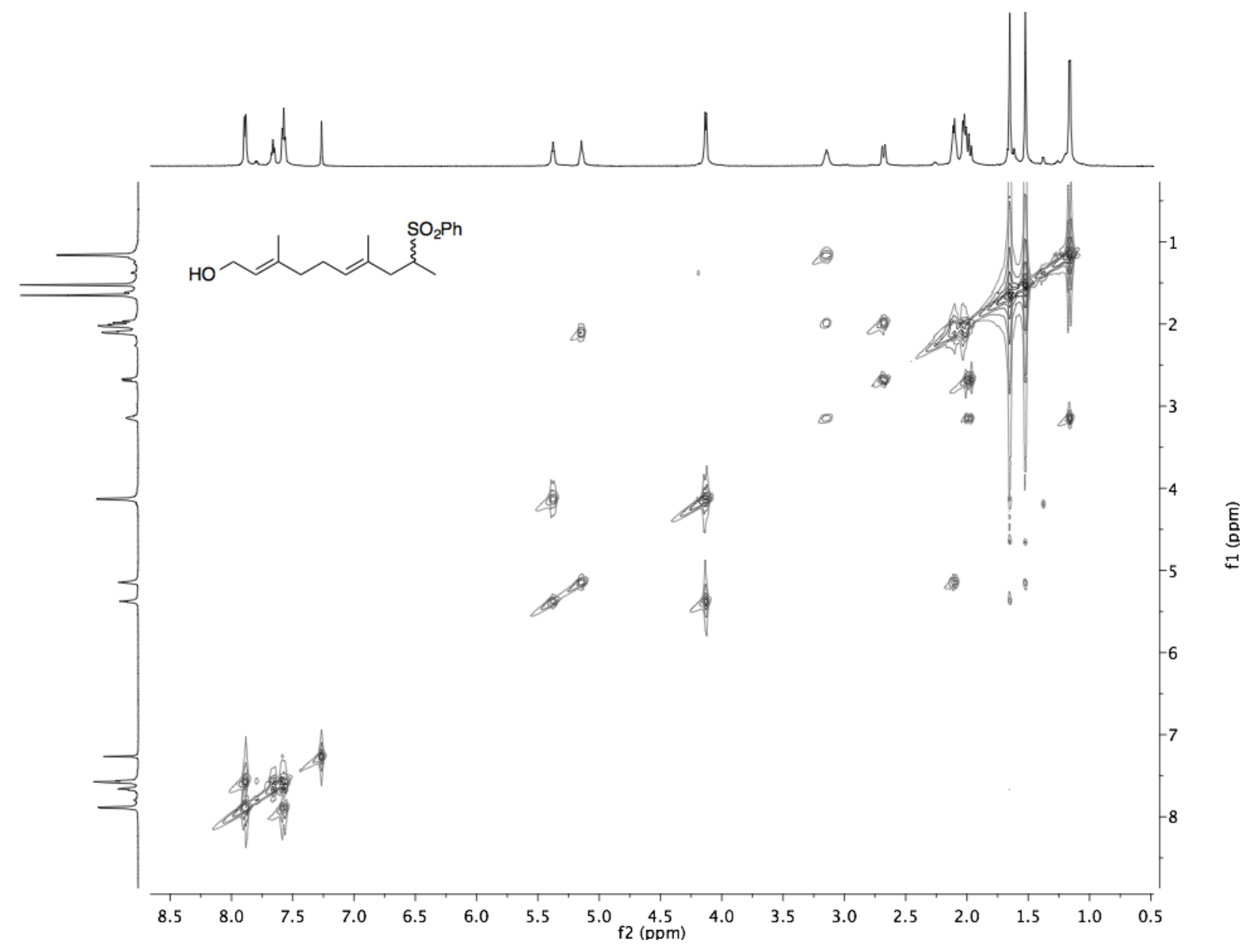

${ }^{1} \mathrm{H}$ NMR Spectrum of $15 \mathrm{~A}$

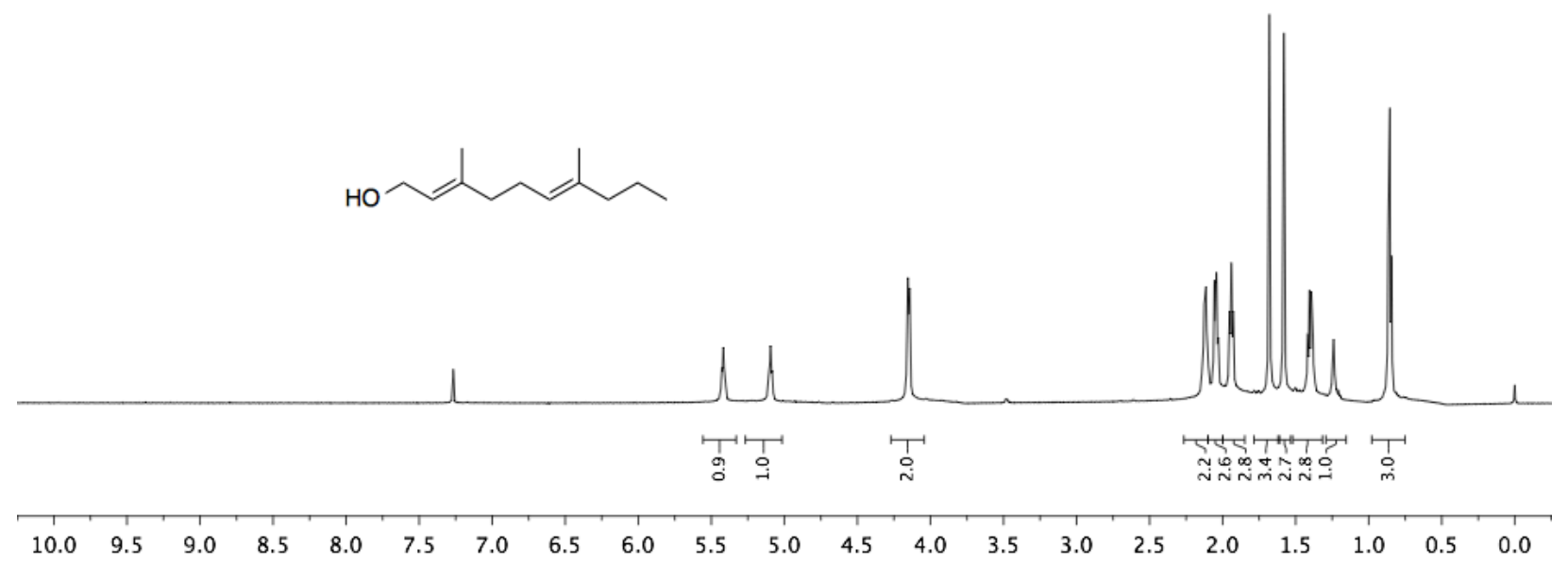


${ }^{13}$ C NMR Spectrum of $15 A$

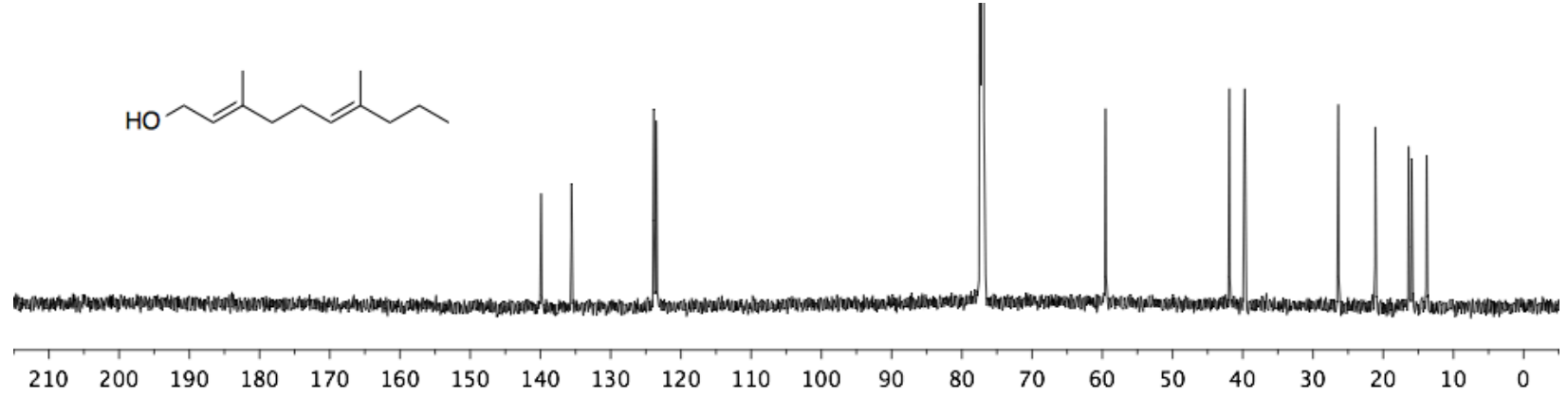

${ }^{1} \mathrm{H}-{ }^{1} \mathrm{H}$ COSY NMR Spectrum of $15 \mathrm{~A}$

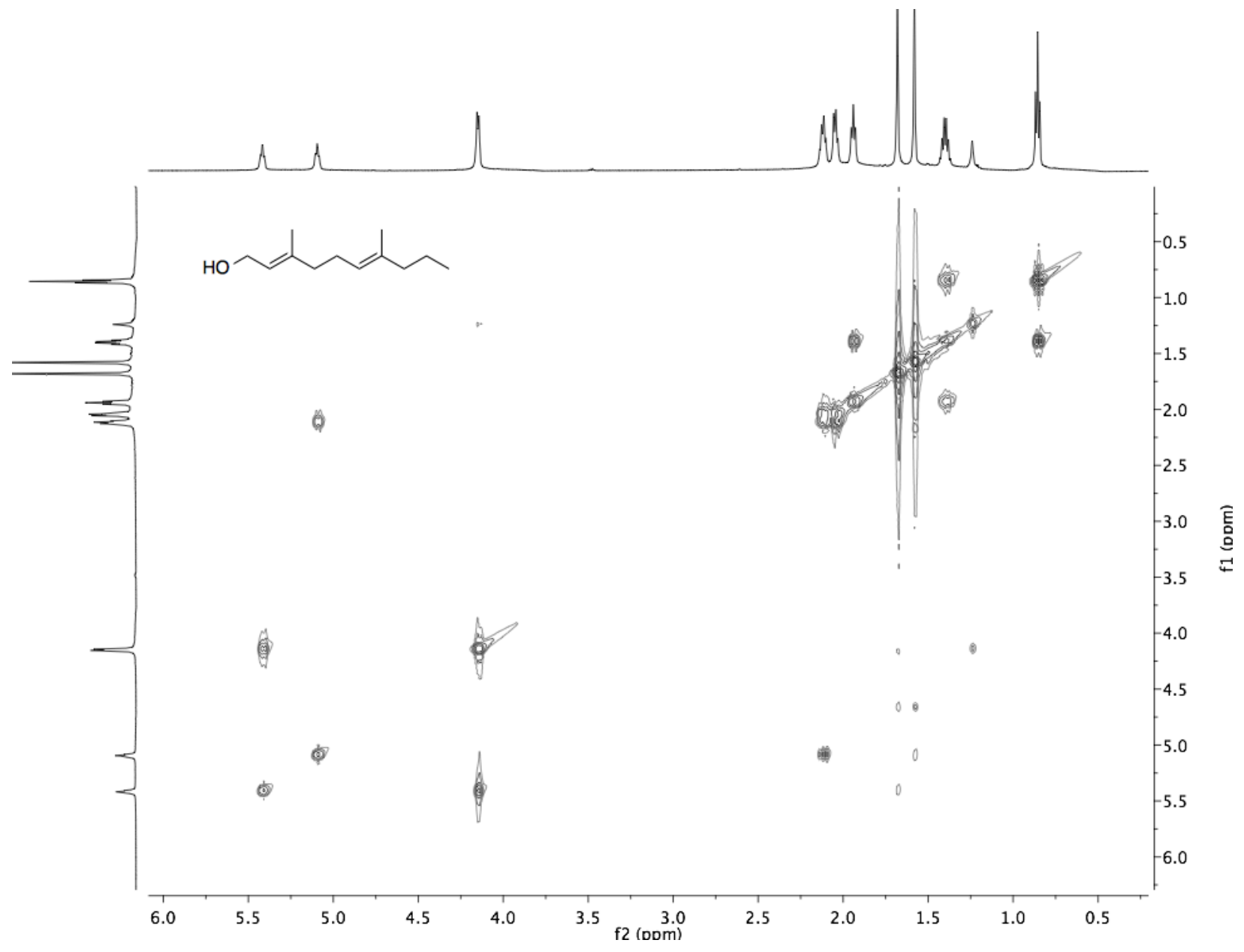


${ }^{1}$ H NMR Spectrum of 16

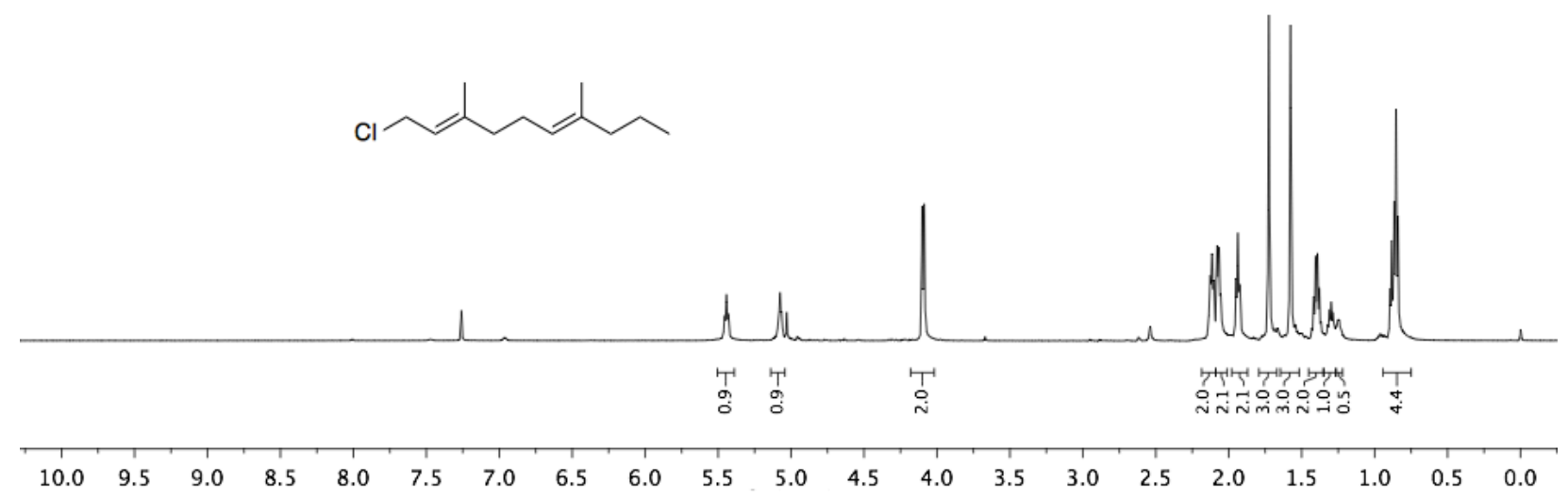

${ }^{13} \mathrm{C}$ NMR Spectrum of 16

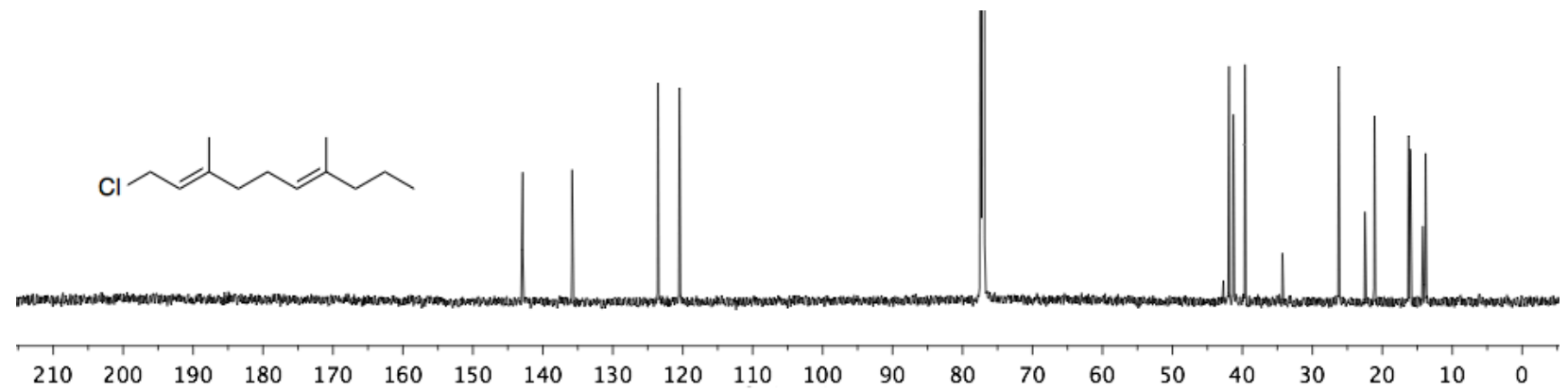




\section{${ }^{1} H-{ }^{1} H$ COSY NMR Spectrum of 16}

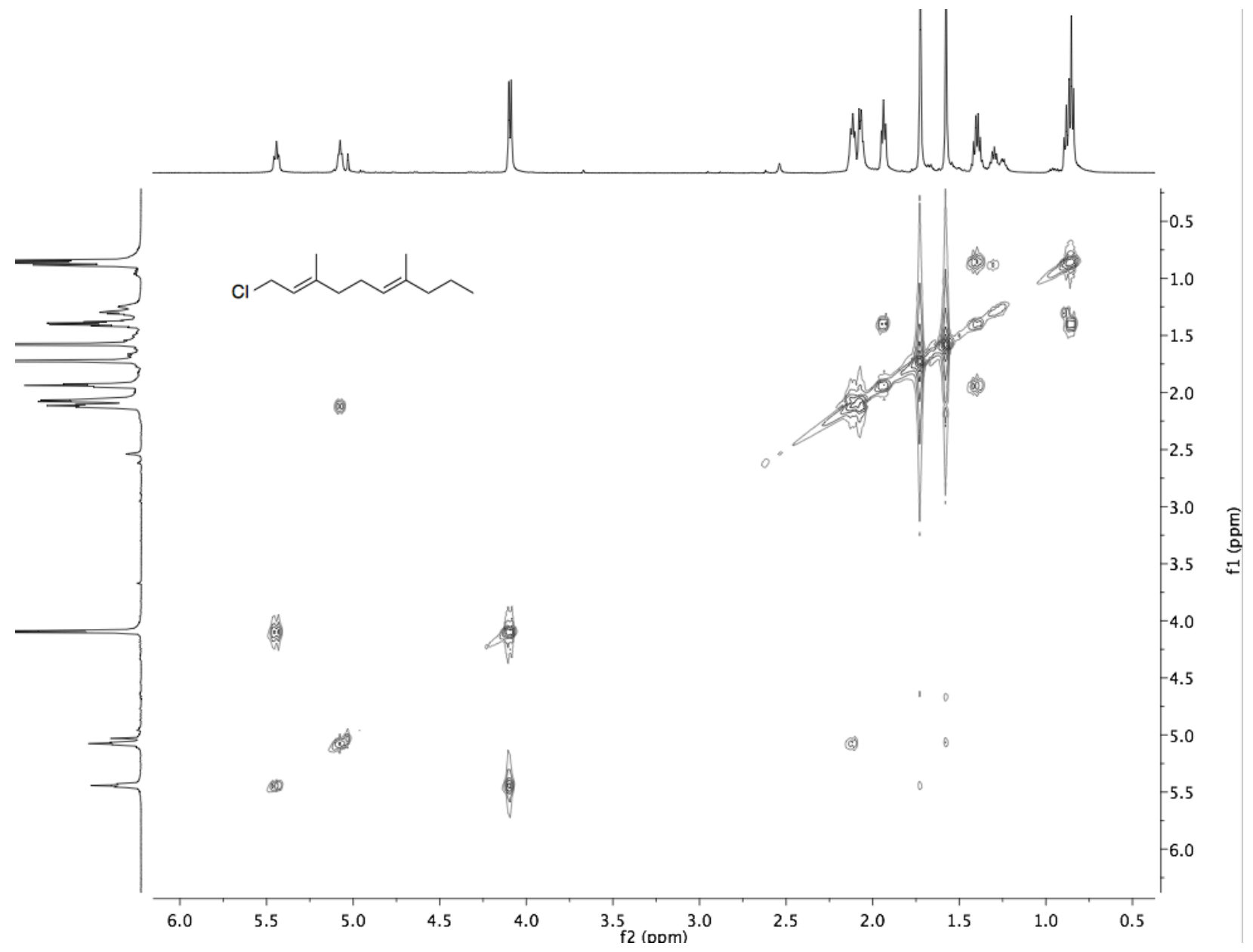

${ }^{1}$ H NMR Spectrum of 19 (a volatile liquid)

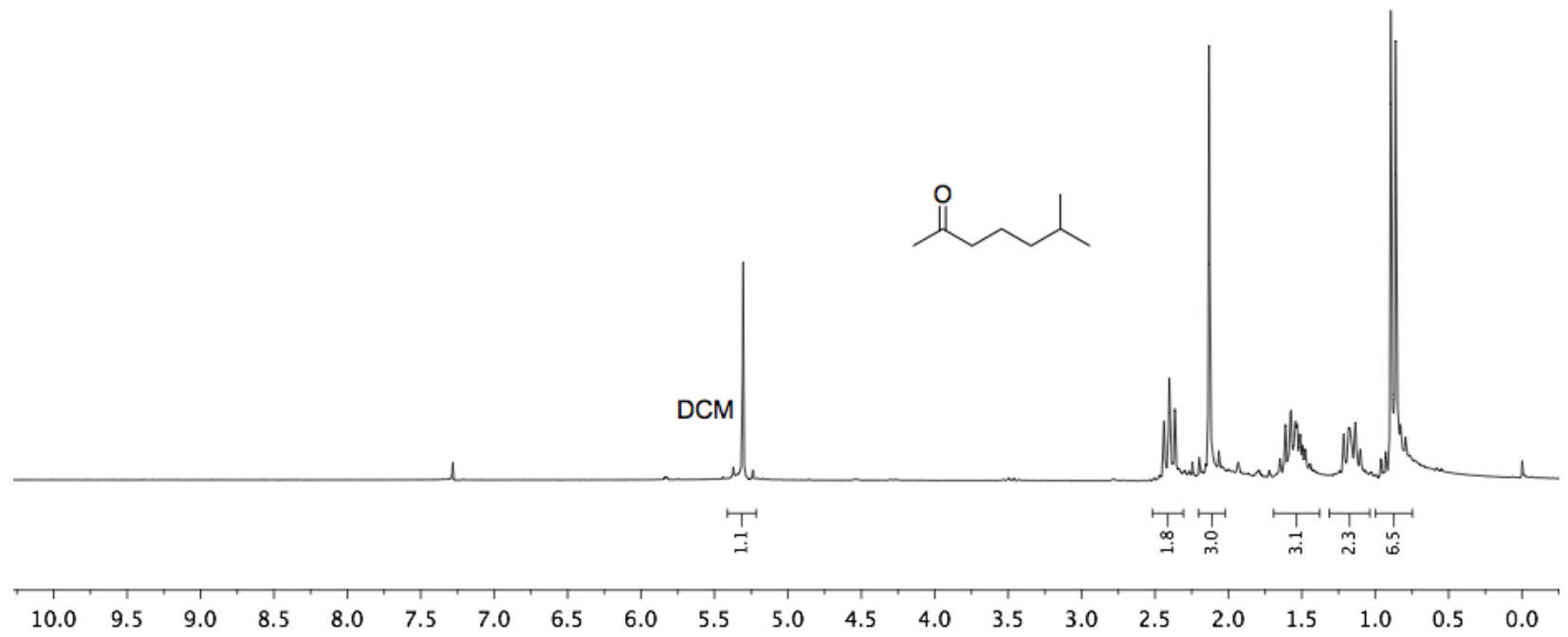


${ }^{1}$ H NMR Spectrum of 20

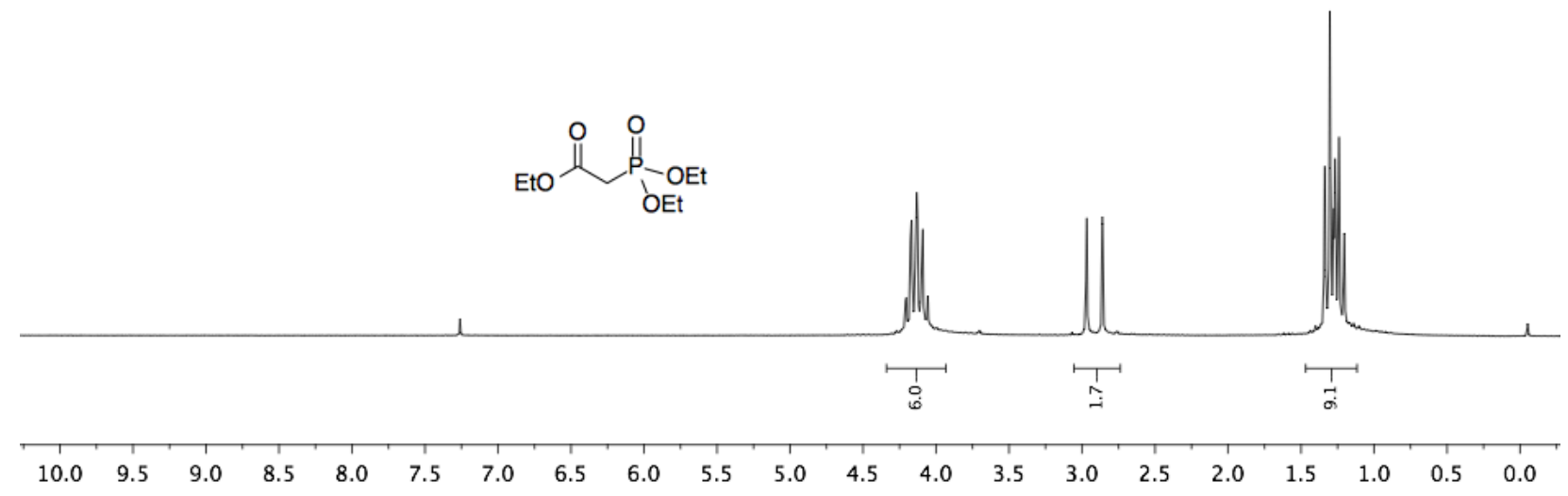

${ }^{1}$ H NMR Spectrum of 21-E

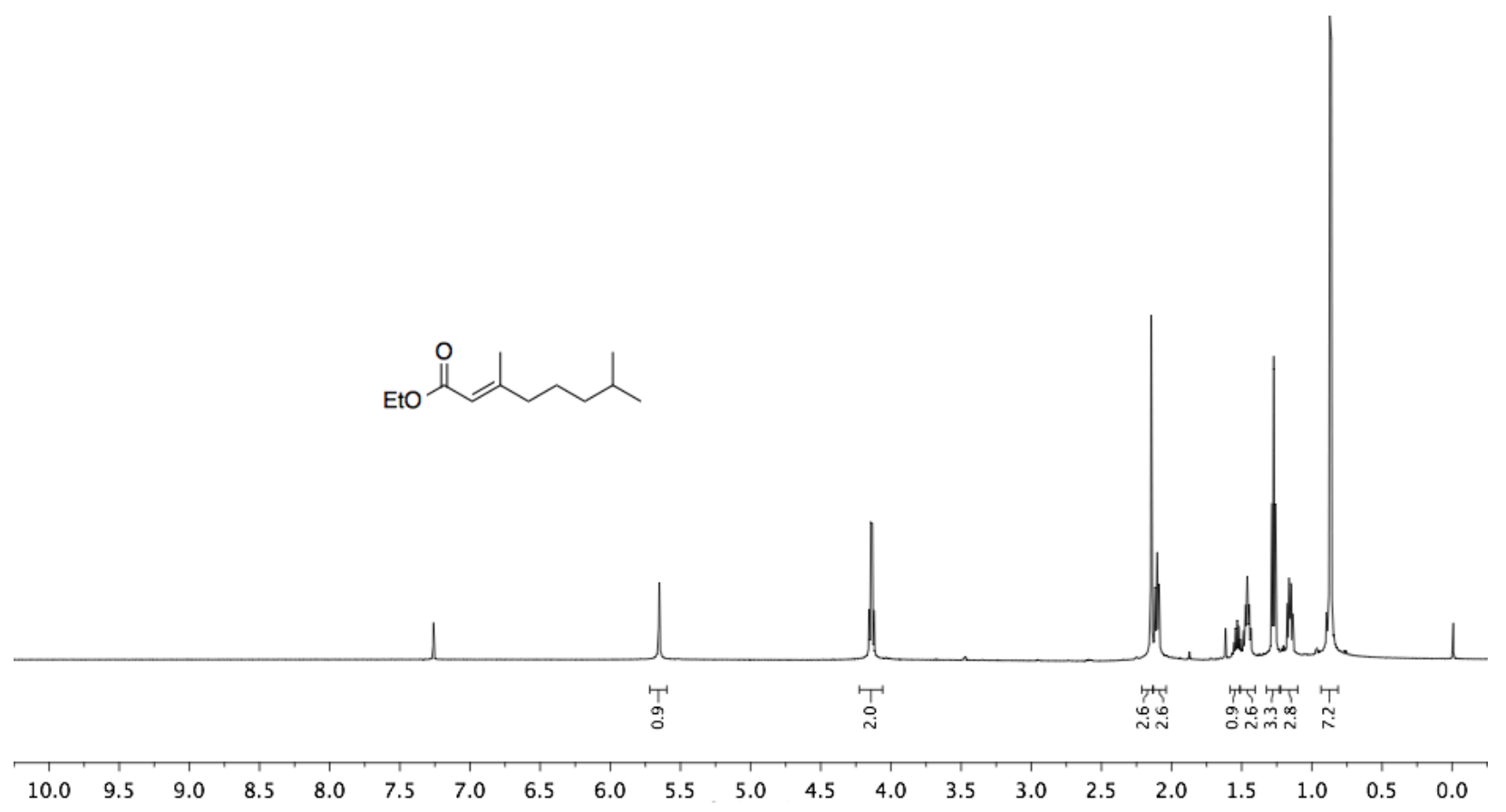


${ }^{13}$ C NMR Spectrum of 21-E

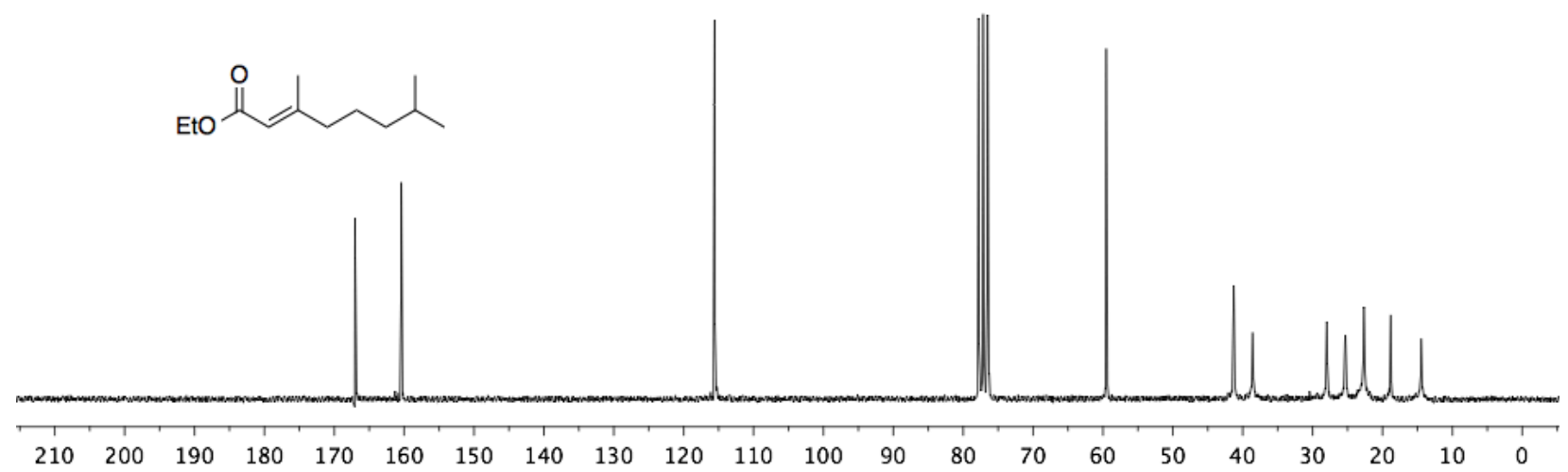

${ }^{1} \mathrm{H}-{ }^{1} \mathrm{H}$ COSY NMR Spectrum of 21-E

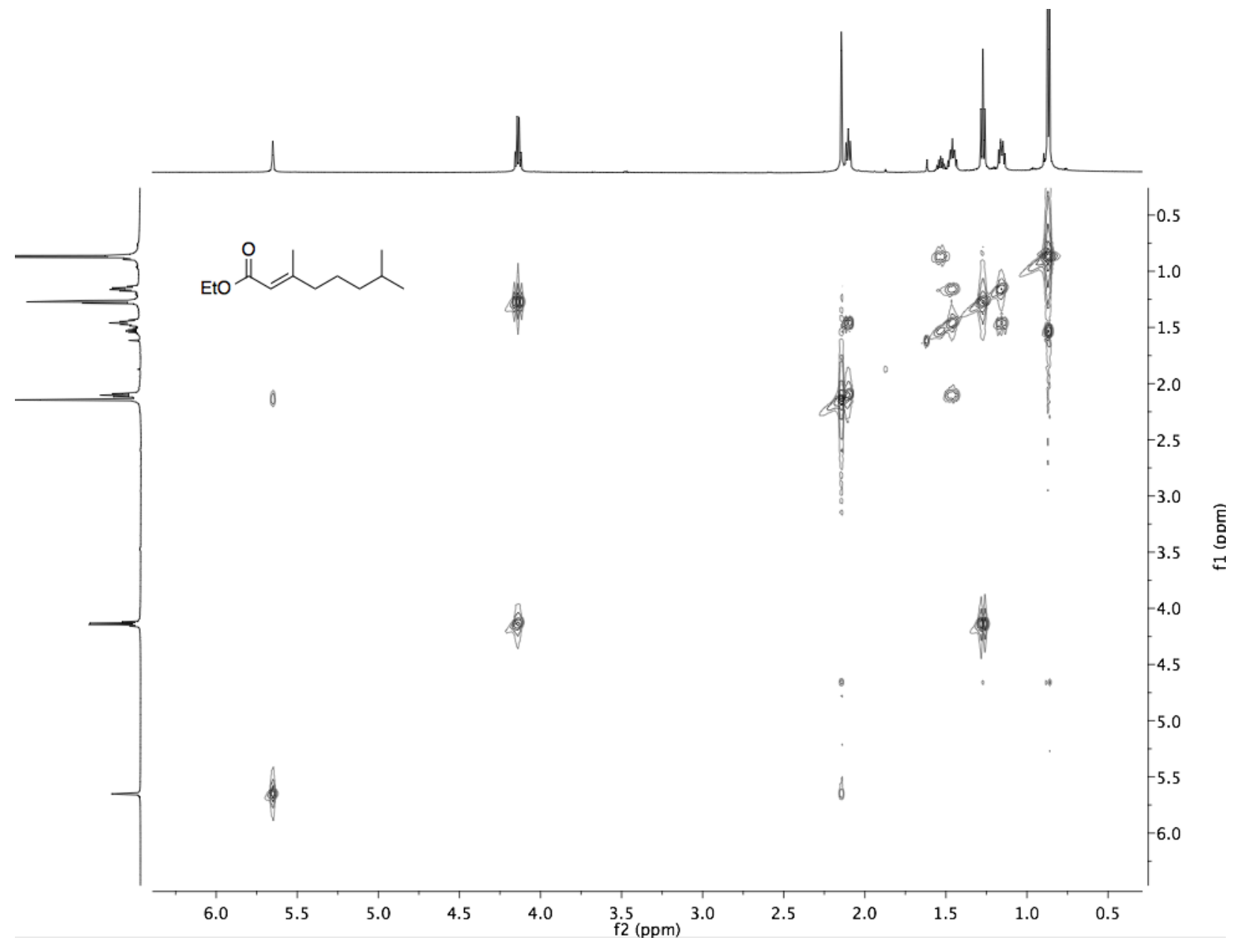


${ }^{1} \mathrm{H}$ NMR Spectrum of 21-Z

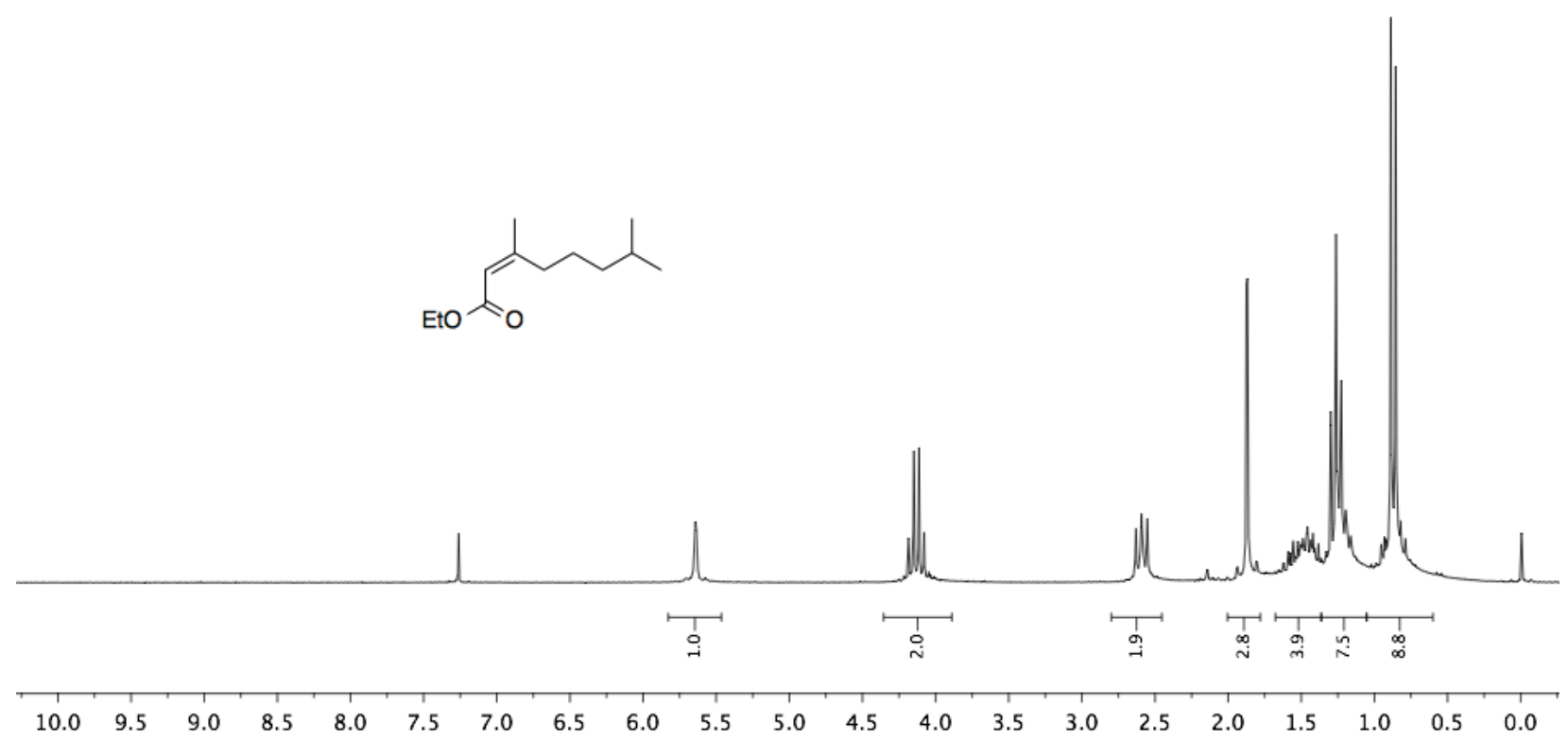

${ }^{1} \mathrm{H}$ NMR Spectrum of $21 \mathrm{~A}$

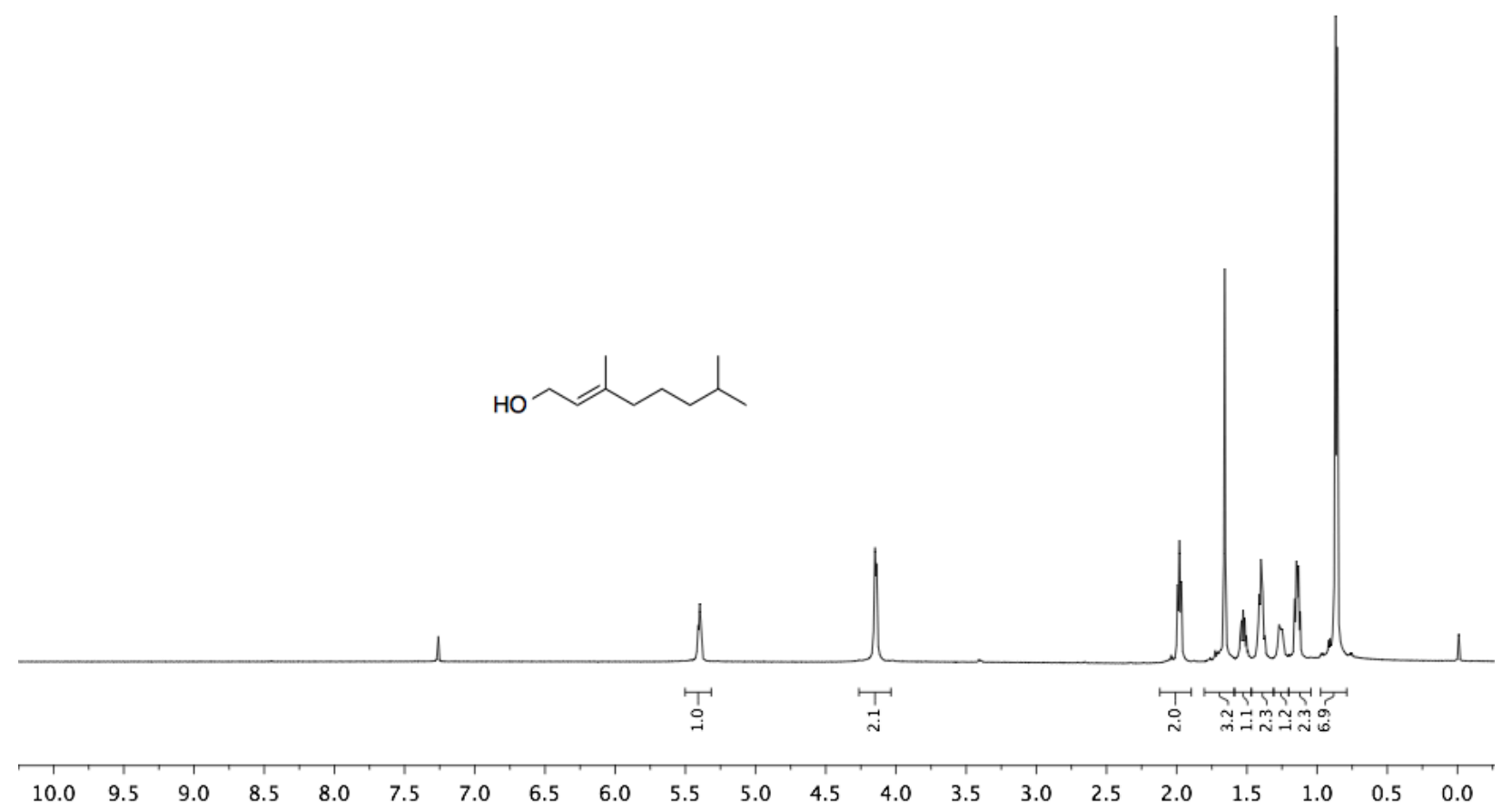


${ }^{13}$ C NMR Spectrum of $21 \mathrm{~A}$

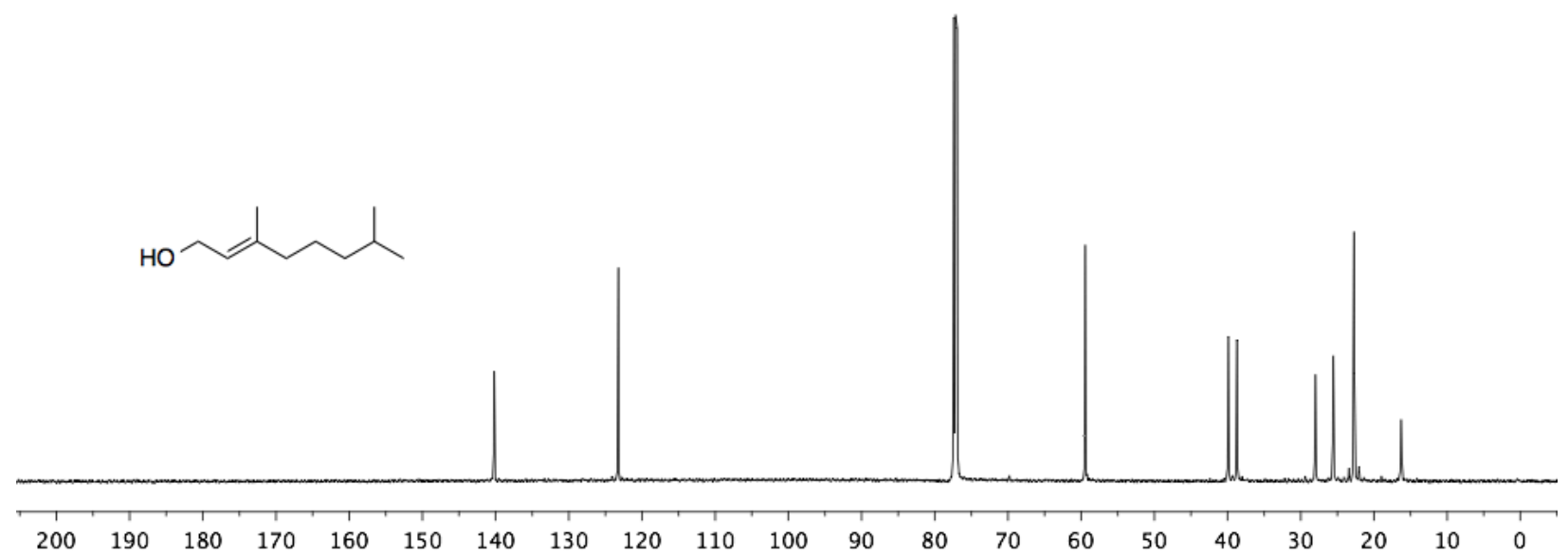

${ }^{1} \mathrm{H}-{ }^{1} \mathrm{H}$ COSY NMR Spectrum of $21 \mathrm{~A}$

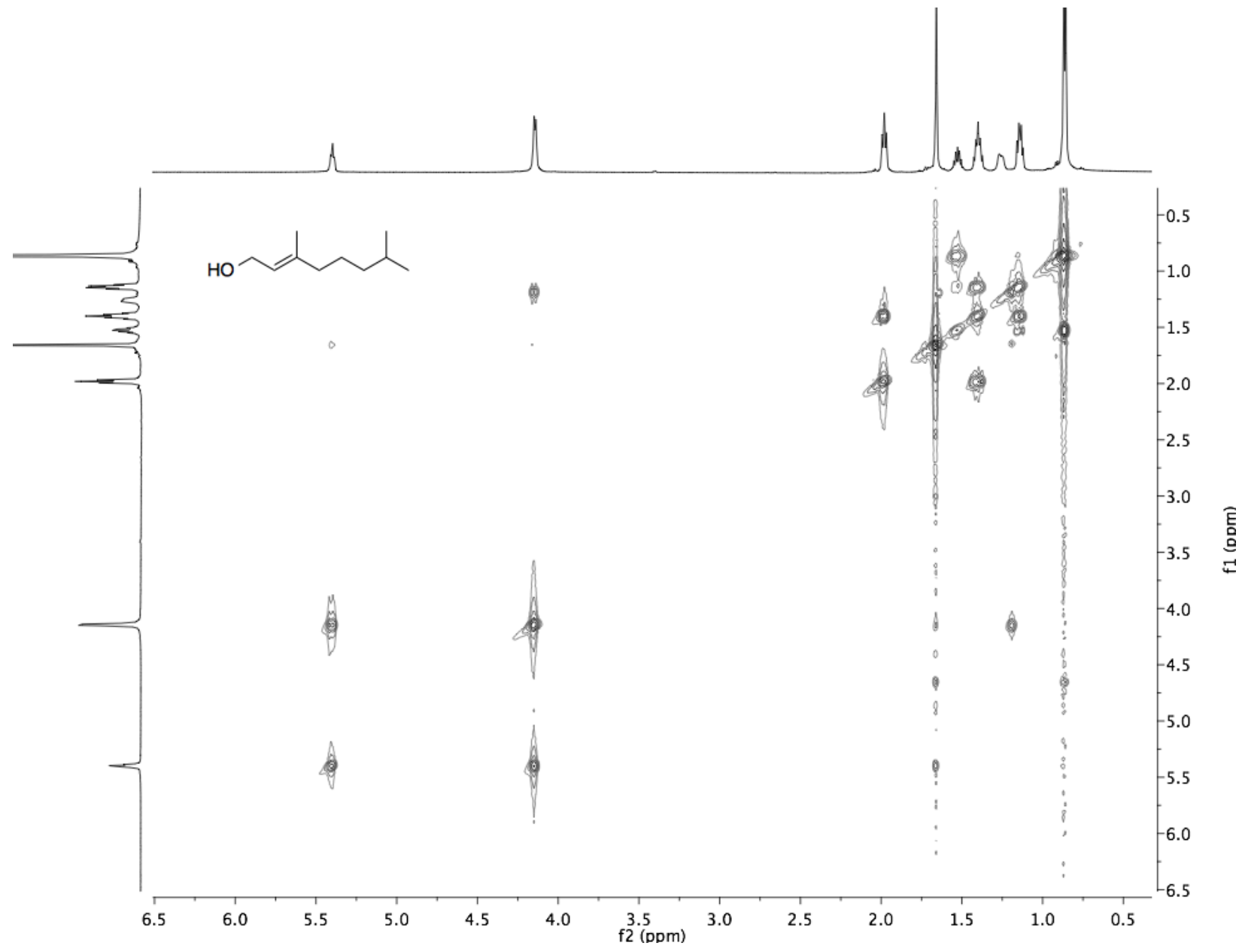


${ }^{1}$ H NMR Spectrum of 22

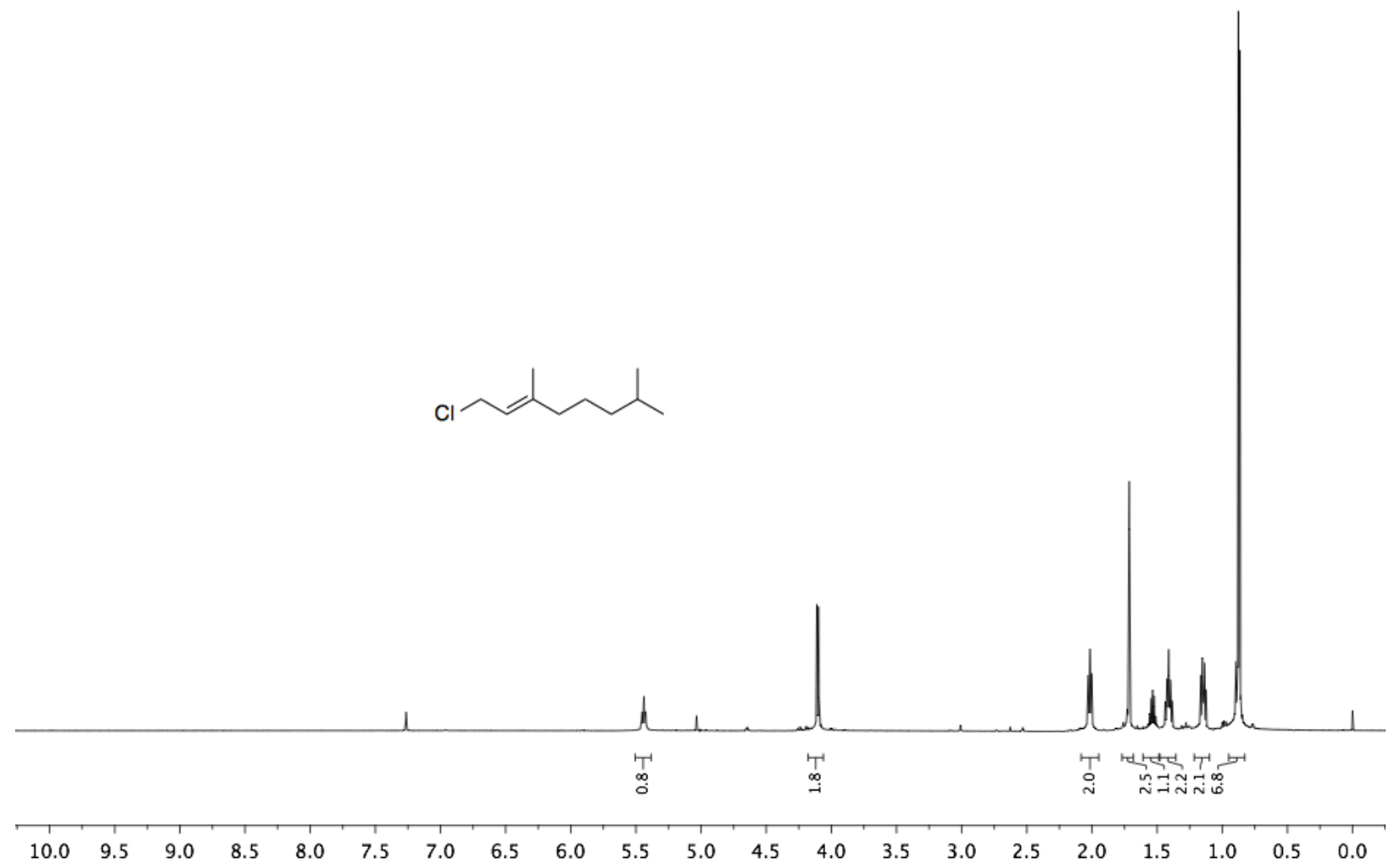

${ }^{13}$ C NMR Spectrum of 22

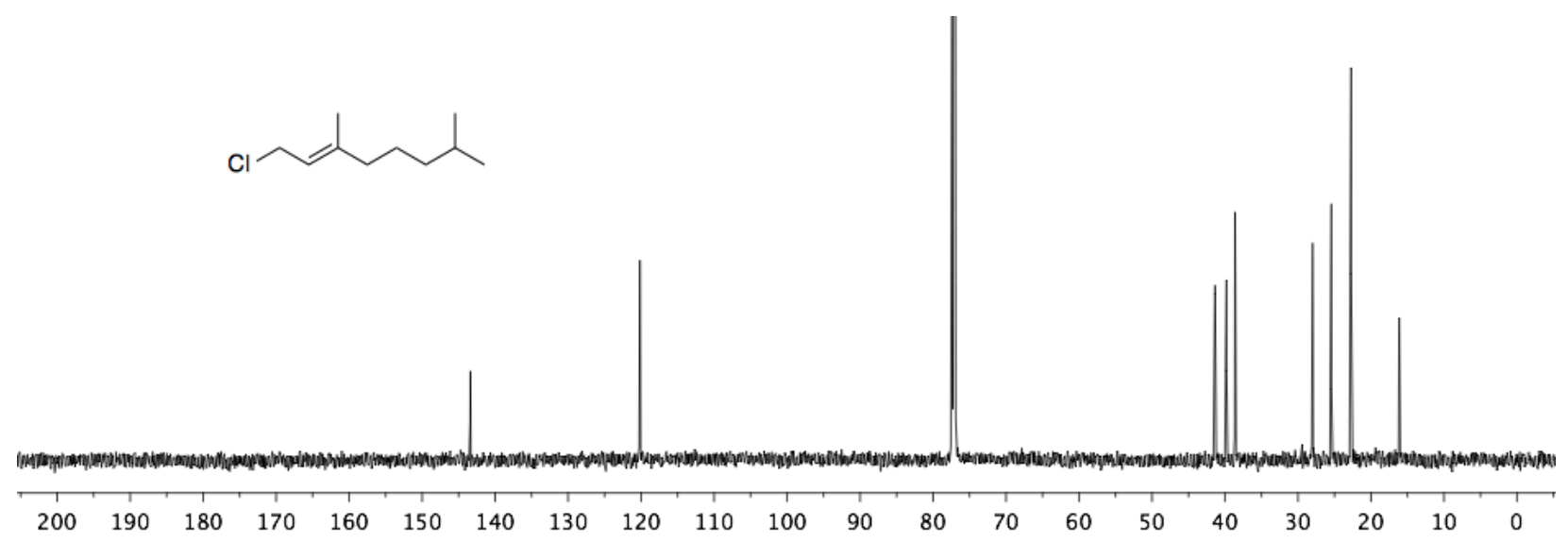




\section{${ }^{1} \mathrm{H}-{ }^{1} \mathrm{H}$ COSY NMR Spectrum of 22}

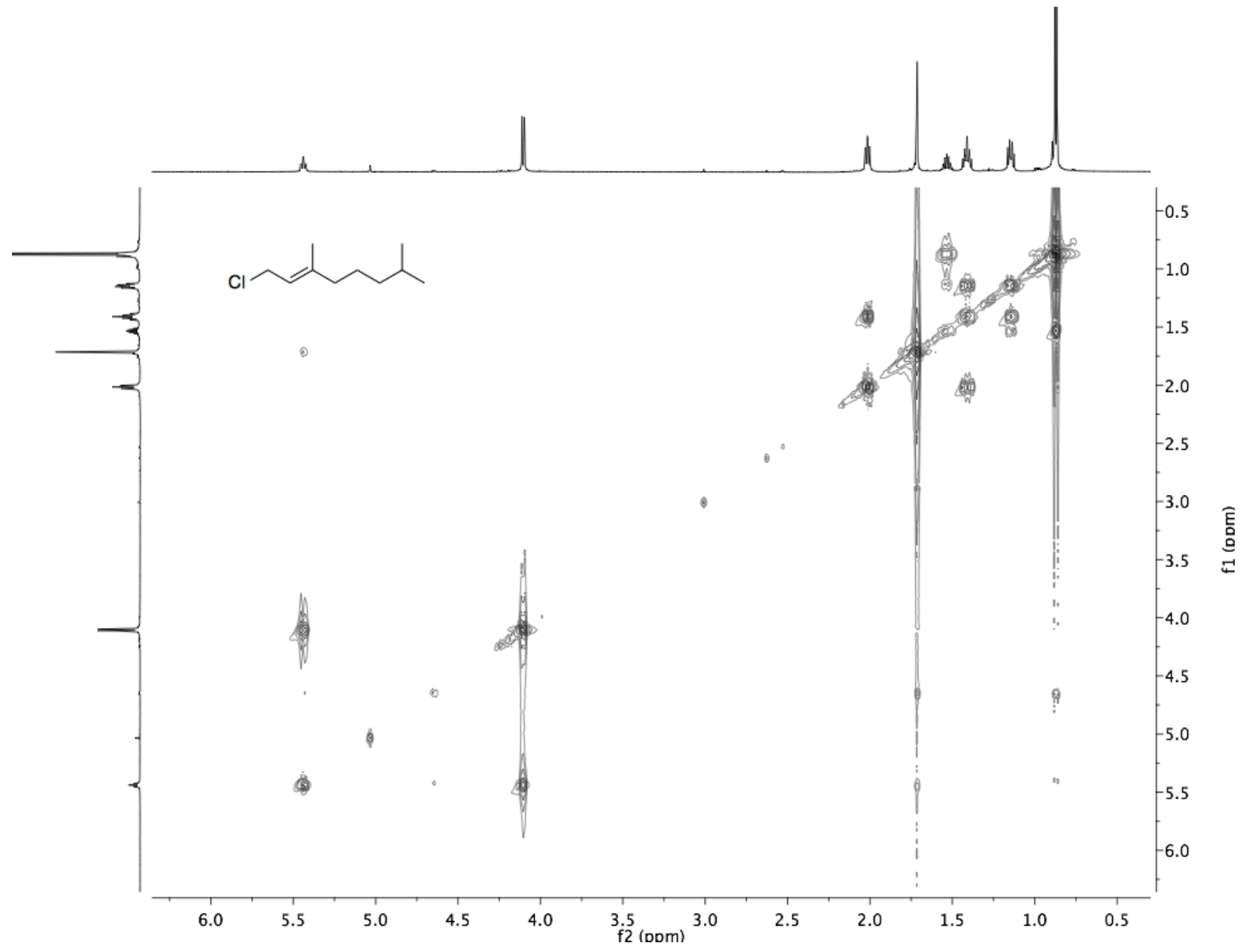

${ }^{1} \mathrm{H}$ NMR Spectrum of $24 \mathrm{~A}$

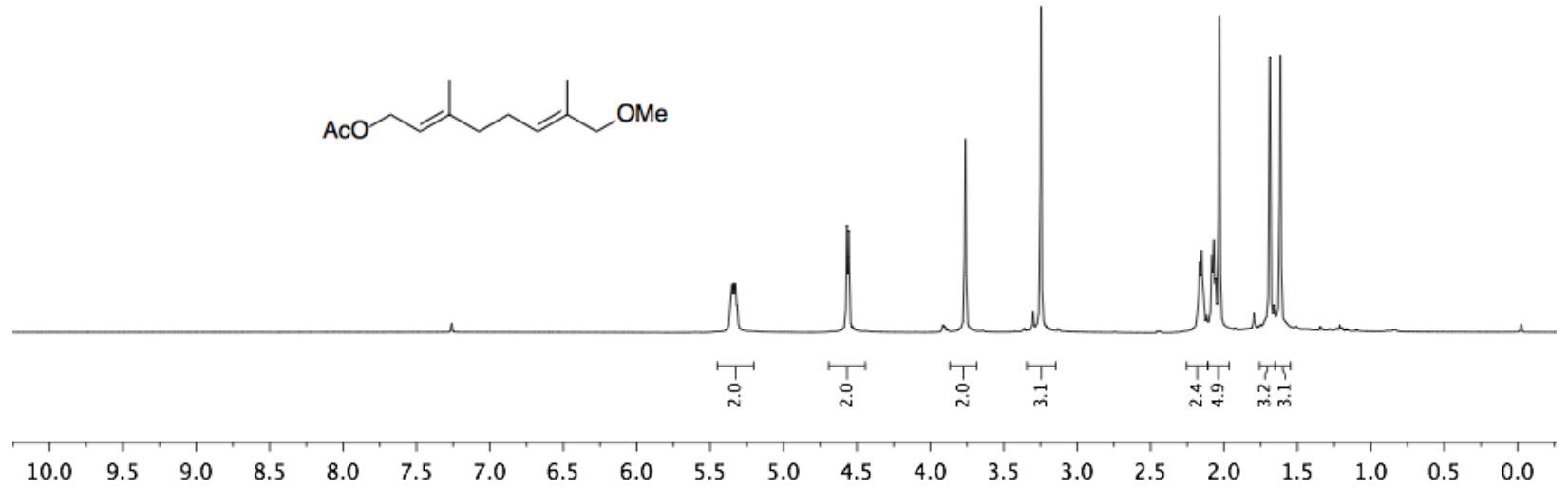


${ }^{13}$ C NMR Spectrum of 24A

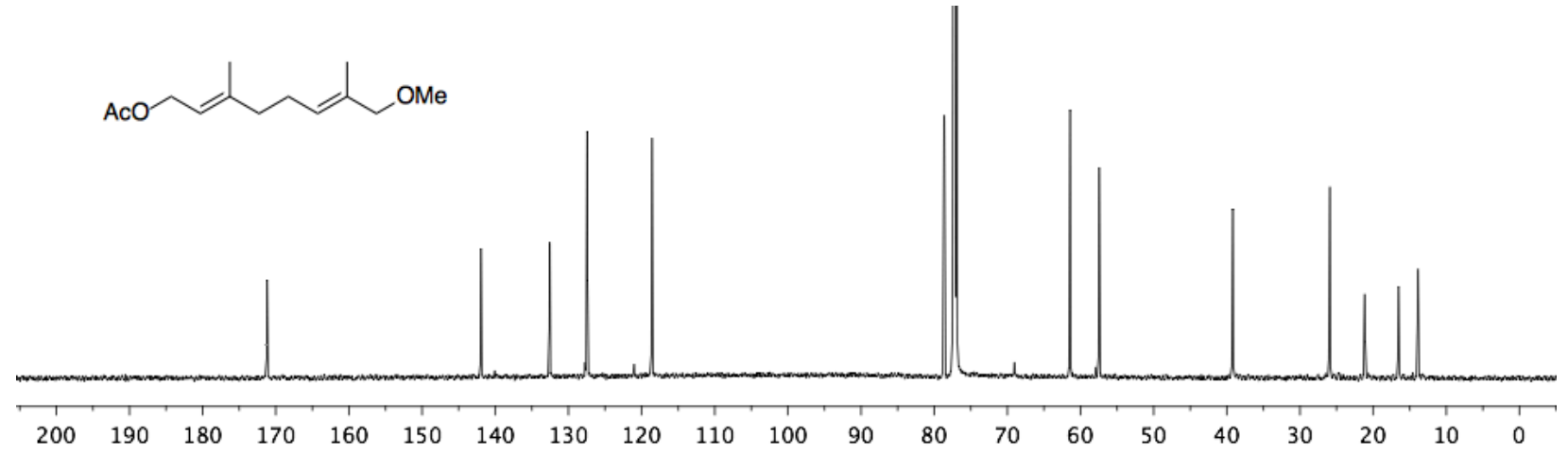

${ }^{1} \mathrm{H}-{ }^{1} \mathrm{H}$ COSY NMR Spectrum of $24 \mathrm{~A}$

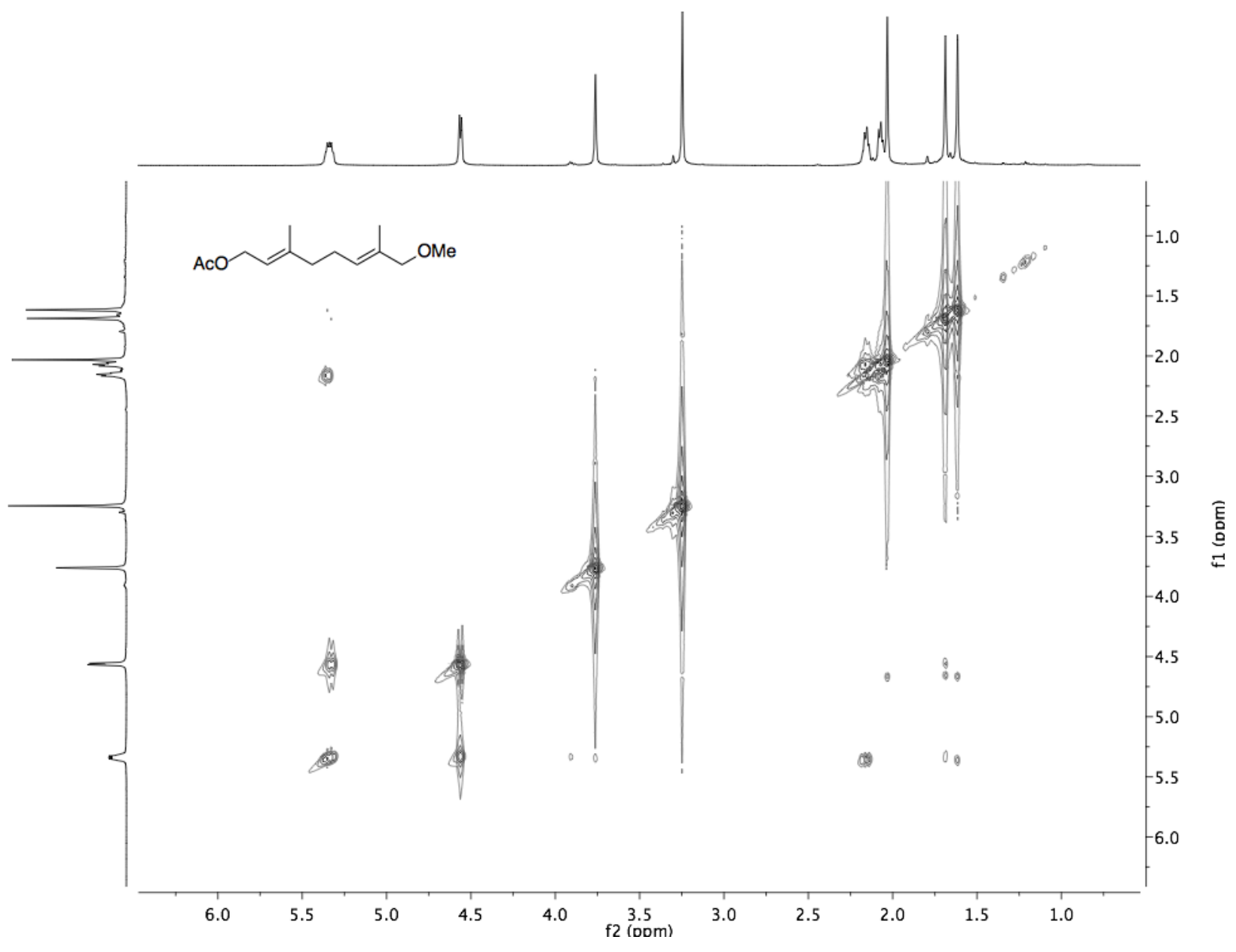


${ }^{1}$ H NMR Spectrum of 24B

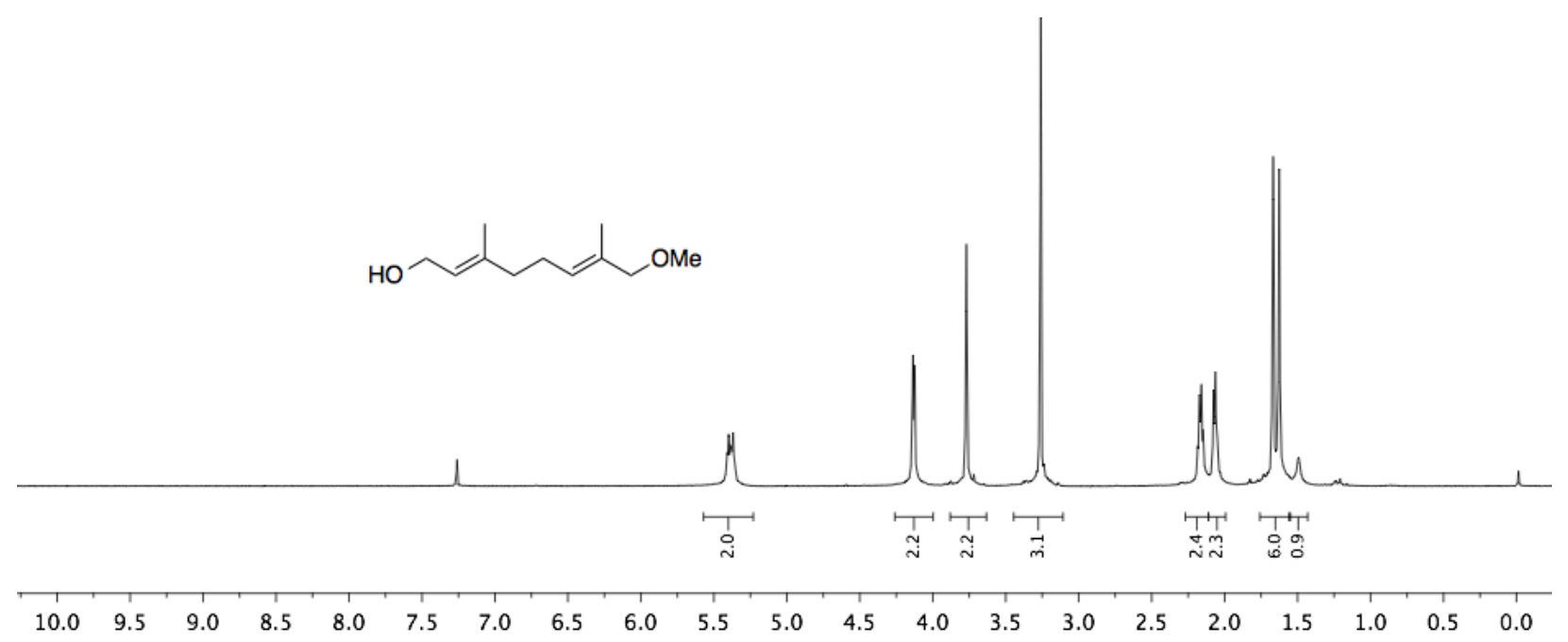

${ }^{13}$ C NMR Spectrum of 24B

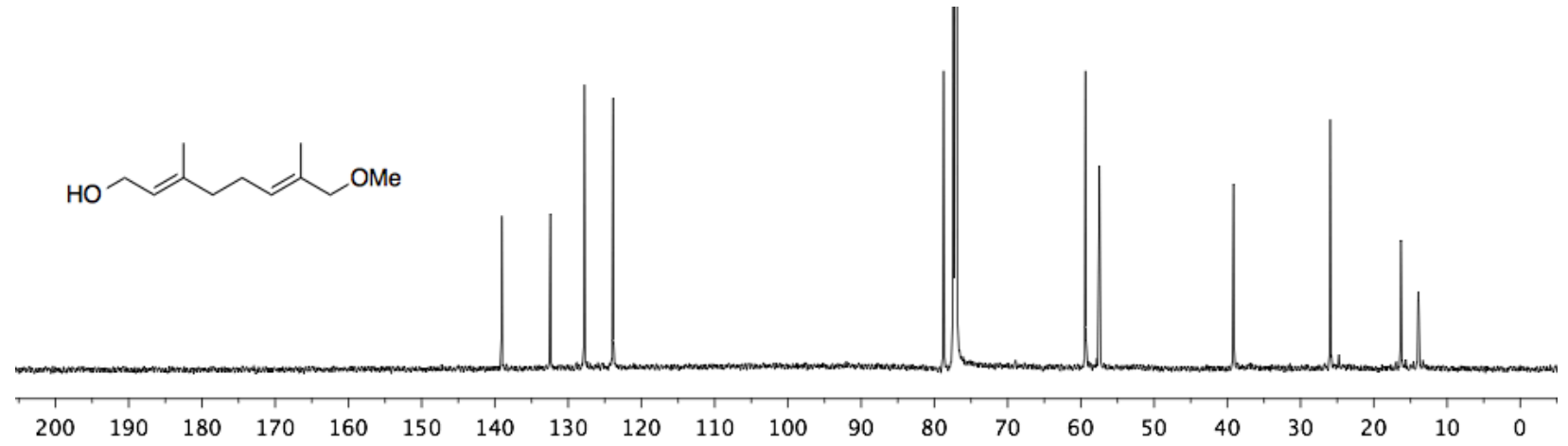


${ }^{1} \mathrm{H}-{ }^{1} \mathrm{H}$ COSY NMR Spectrum of 24B

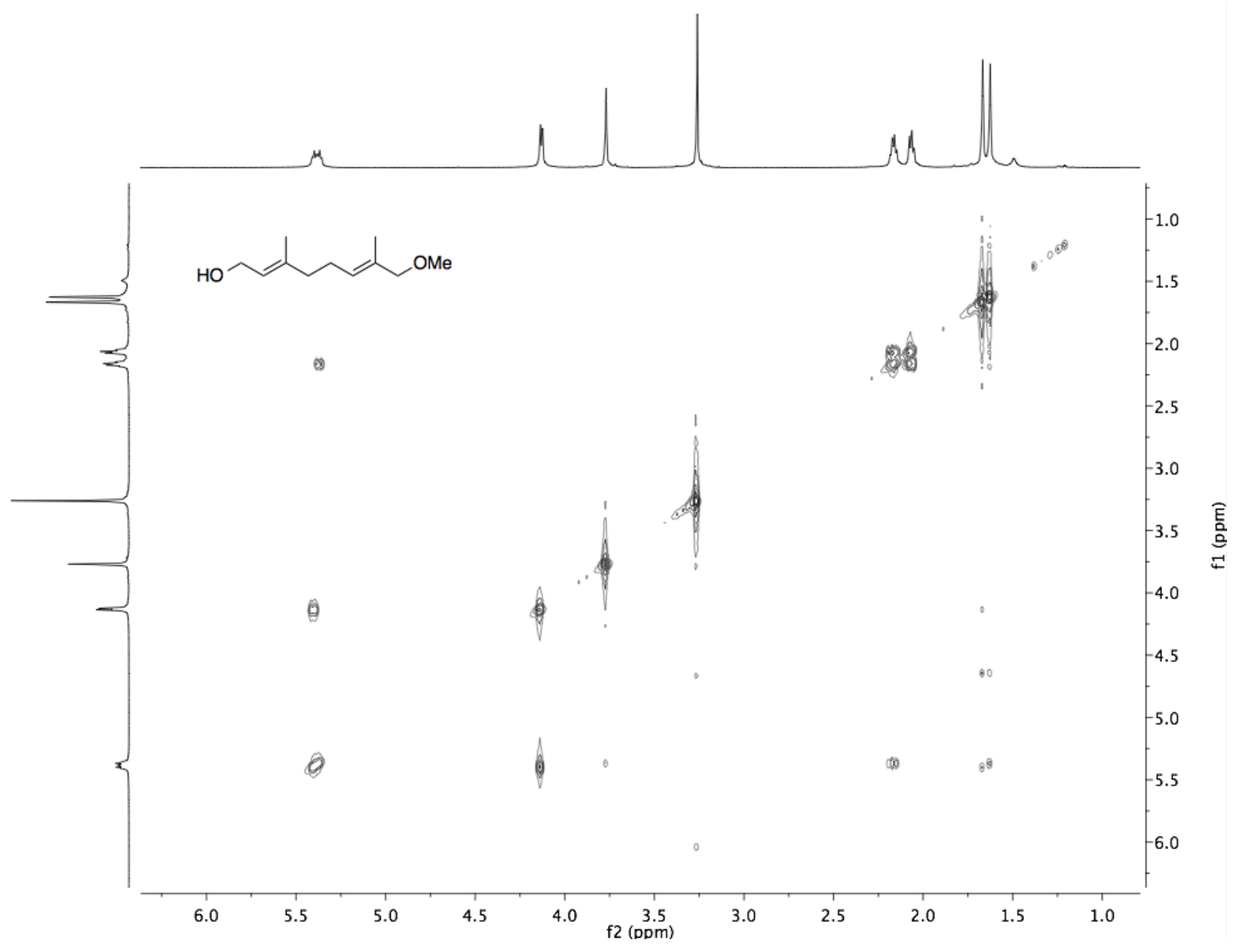

${ }^{1}$ H NMR Spectrum of 24

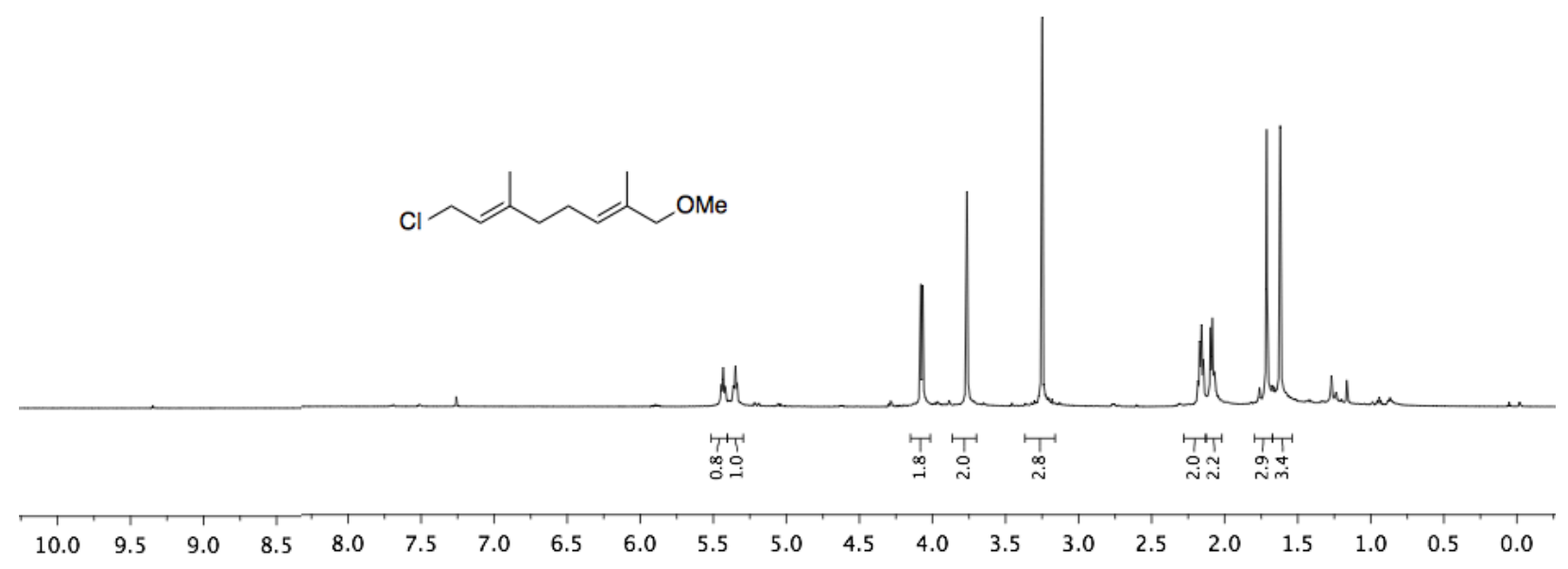


${ }^{13}$ C NMR Spectrum of 24

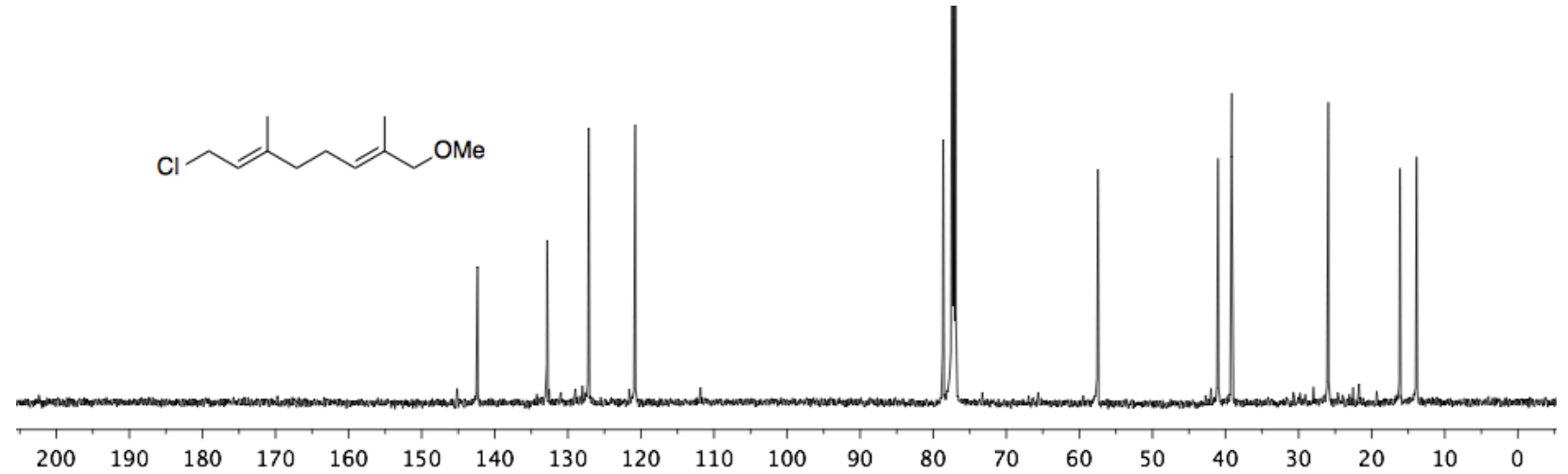

${ }^{1} \mathrm{H}-{ }^{1} \mathrm{H}$ COSY NMR Spectrum of 24

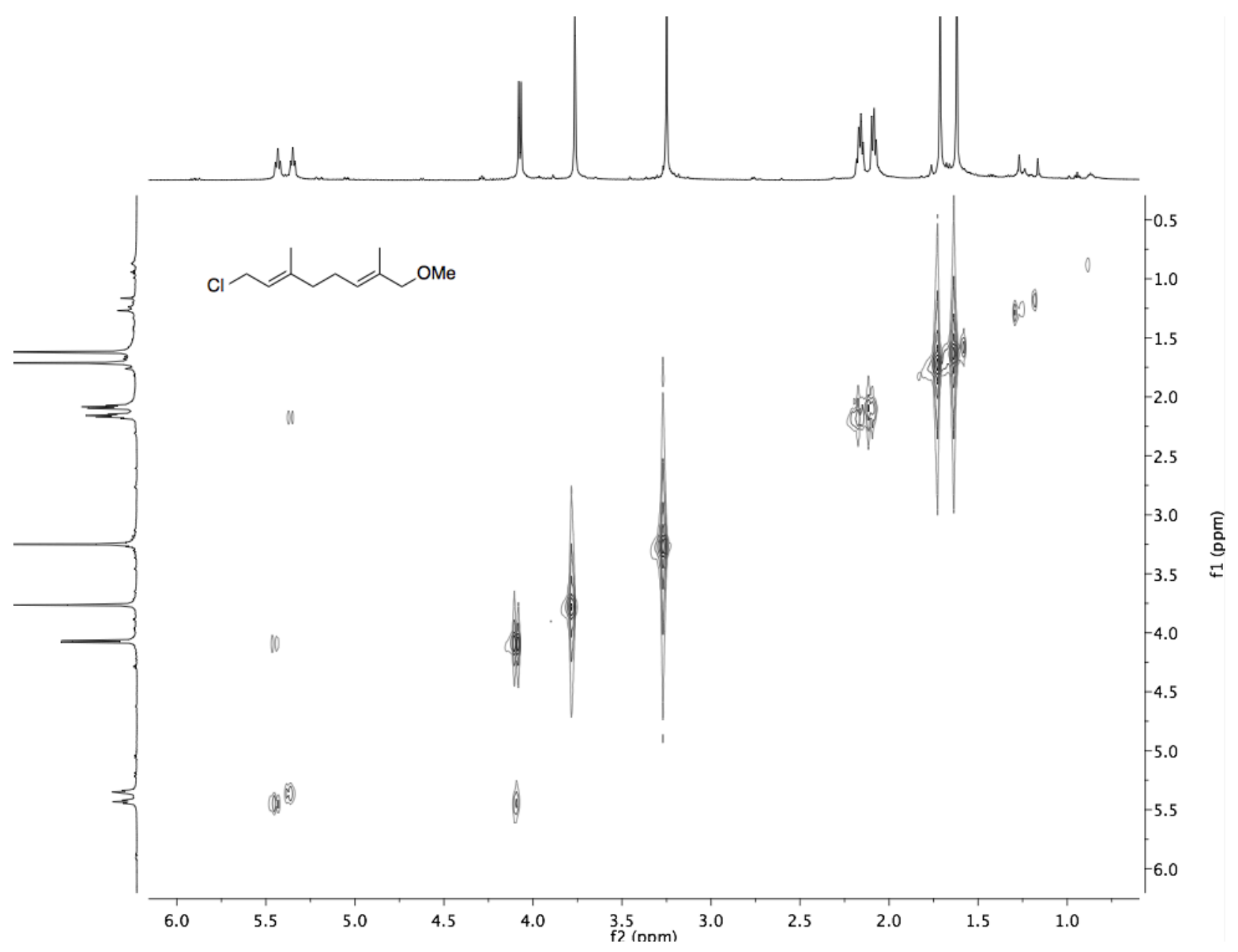




\section{Atomic Coordinates of Calculated Structures}

\subsection{Conformations of Geranyl Chloride Reactant}

This structure was the input for the geranyl chloride conformation search in Molecular Operating Environment.

\begin{tabular}{|c|c|c|c|c|c|}
\hline HETATM & $1 \mathrm{C}$ & * & 0 & $\begin{array}{lllll}0.171 & 0.482 & -0.100 & 1.00 & 0.00\end{array}$ & $\mathrm{C}$ \\
\hline HETATM & $2 \mathrm{C} 1$ & $*$ & 0 & $\begin{array}{lllll}1.626 & 0.063 & -0.156 & 1.00 & 0.00\end{array}$ & $\mathrm{C}$ \\
\hline HETATM & 3 CL2 & * & 0 & $\begin{array}{lllll}-0.266 & 0.253 & 1.618 & 1.00 & 0.00\end{array}$ & CL \\
\hline HETATM & $4 \mathrm{C}$ & * & 0 & $\begin{array}{lllll}2.080 & -1.085 & -0.689 & 1.00 & 0.00\end{array}$ & $\mathrm{C}$ \\
\hline HETATM & $5 \mathrm{C} 1$ & $*$ & 0 & $\begin{array}{lllll}3.569 & -1.396 & -0.705 & 1.00 & 0.00\end{array}$ & $\mathrm{C}$ \\
\hline HETATM & $6 \mathrm{C} 11$ & * & 0 & $\begin{array}{lllll}4.060 & -1.107 & -2.136 & 1.00 & 0.00\end{array}$ & $\mathrm{C}$ \\
\hline HETATM & $7 \mathrm{C} 112$ & * & 0 & $\begin{array}{lllll}5.549 & -1.406 & -2.127 & 1.00 & 0.00\end{array}$ & $\mathrm{C}$ \\
\hline HETATM & $8 \mathrm{C} 2$ & $*$ & 0 & $\begin{array}{lllll}1.159 & -2.122 & -1.314 & 1.00 & 0.00\end{array}$ & $\mathrm{C}$ \\
\hline HETATM & $9 \mathrm{C}$ & * & 0 & $\begin{array}{lllll}6.119 & -2.544 & -2.563 & 1.00 & 0.00\end{array}$ & $\mathrm{C}$ \\
\hline HETATM & $10 \mathrm{C} 1$ & * & 0 & $\begin{array}{lllll}7.625 & -2.731 & -2.523 & 1.00 & 0.00\end{array}$ & $\mathrm{C}$ \\
\hline HETATM & $11 \mathrm{C} 2$ & $*$ & 0 & $\begin{array}{lllll}5.329 & -3.711 & -3.134 & 1.00 & 0.00\end{array}$ & $\mathrm{C}$ \\
\hline HETATM & $12 \mathrm{H} 1$ & $*$ & 0 & $\begin{array}{lllll}2.296 & 0.763 & 0.273 & 1.00 & 0.00\end{array}$ & $\mathrm{H}$ \\
\hline HETATM & $13 \mathrm{H} 3$ & $*$ & 0 & $\begin{array}{lllll}-0.468 & -0.158 & -0.690 & 1.00 & 0.00\end{array}$ & $\mathrm{H}$ \\
\hline HETATM & $14 \mathrm{H} 4$ & * & 0 & $\begin{array}{lllll}0.075 & 1.535 & -0.327 & 1.00 & 0.00\end{array}$ & $\mathrm{H}$ \\
\hline HETATM & $15 \mathrm{H} 111$ & $*$ & 0 & $\begin{array}{lllll}3.545 & -1.743 & -2.842 & 1.00 & 0.00\end{array}$ & $\mathrm{H}$ \\
\hline HETATM & $16 \mathrm{H} 112$ & $*$ & 0 & $\begin{array}{lllll}6.142 & -0.623 & -1.728 & 1.00 & 0.00\end{array}$ & \\
\hline HETATM & $17 \mathrm{H} 113$ & $*$ & 0 & $\begin{array}{lllll}3.922 & -0.058 & -2.357 & 1.00 & 0.00\end{array}$ & $\mathrm{H}$ \\
\hline HETATM & $18 \mathrm{H} 12$ & $*$ & 0 & $\begin{array}{lllll}3.725 & -2.439 & -0.464 & 1.00 & 0.00\end{array}$ & $\mathrm{H}$ \\
\hline HETATM & $19 \mathrm{H} 13$ & $*$ & 0 & $\begin{array}{lllll}4.085 & -0.738 & -0.017 & 1.00 & 0.00\end{array}$ & $\mathrm{H}$ \\
\hline HETATM & $20 \mathrm{H} 21$ & $*$ & 0 & $\begin{array}{lllll}0.776 & -1.700 & -2.233 & 1.00 & 0.00\end{array}$ & $\mathrm{H}$ \\
\hline HETATM & $21 \mathrm{H} 22$ & $*$ & 0 & $\begin{array}{lllll}0.366 & -2.327 & -0.608 & 1.00 & 0.00\end{array}$ & $\mathrm{H}$ \\
\hline HETATM & $22 \mathrm{H} 23$ & $*$ & 0 & $\begin{array}{lllll}1.728 & -3.021 & -1.510 & 1.00 & 0.00\end{array}$ & $\mathrm{H}$ \\
\hline HETATM & $23 \mathrm{H} 11$ & $*$ & 0 & $\begin{array}{lllll}7.951 & -2.930 & -3.535 & 1.00 & 0.00\end{array}$ & $\mathrm{H}$ \\
\hline HETATM & $24 \mathrm{H} 12$ & $*$ & 0 & $\begin{array}{lllll}7.829 & -3.569 & -1.870 & 1.00 & 0.00\end{array}$ & $\mathrm{H}$ \\
\hline HETATM & $25 \mathrm{H} 13$ & $*$ & 0 & $\begin{array}{lllll}8.078 & -1.826 & -2.143 & 1.00 & 0.00\end{array}$ & $\mathrm{H}$ \\
\hline HETATM & $26 \mathrm{H} 21$ & $*$ & 0 & $\begin{array}{lllll}5.963 & -4.586 & -3.132 & 1.00 & 0.00\end{array}$ & $\mathrm{H}$ \\
\hline HETATM & $27 \mathrm{H} 22$ & $*$ & 0 & $\begin{array}{lllll}5.044 & -3.441 & -4.141 & 1.00 & 0.00\end{array}$ & $\mathrm{H}$ \\
\hline HETATM & $28 \mathrm{H} 23$ & $*$ & 0 & $\begin{array}{lllll}4.466 & -3.875 & -2.504 & 1.00 & 0.00\end{array}$ & $\mathrm{H}$ \\
\hline
\end{tabular}

These structures are outputs from the conformation search in Molecular Operating Environment without further refinement.

Conformation \#1

$\begin{array}{llllllll}\text { HETATM } & 1 & \mathrm{C} & 0 & 2.325 & -1.754 & -4.416 & \mathrm{C} \\ \text { HETATM } & 2 & \mathrm{C} & 0 & 2.428 & -1.099 & -3.053 & \mathrm{C} \\ \text { HETATM } & 3 & \mathrm{H} & 0 & 1.762 & -0.284 & -2.916 & \mathrm{H} \\ \text { HETATM } & 4 & \mathrm{C} & 0 & 3.270 & -1.473 & -2.074 & \mathrm{C} \\ \text { HETATM } & 5 & \mathrm{C} & 0 & 3.267 & -0.750 & -0.735 & \mathrm{C} \\ \text { HETATM } & 6 & \mathrm{C} & 0 & 2.461 & -1.637 & 0.230 & \mathrm{C} \\ \text { HETATM } & 7 & \mathrm{H} & 0 & 1.444 & -1.723 & -0.130 & \mathrm{H} \\ \text { HETATM } & 8 & \mathrm{C} & 0 & 2.454 & -0.894 & 1.554 & \mathrm{C} \\ \text { HETATM } & 9 & \mathrm{H} & 0 & 1.732 & -0.118 & 1.608 & \mathrm{H} \\ \text { HETATM } & 10 & \mathrm{C} & 0 & 3.269 & -1.148 & 2.592 & \mathrm{C} \\ \text { HETATM } & 11 \mathrm{C} & 0 & 3.165 & -0.352 & 3.878 & \mathrm{C} \\ \text { HETATM } & 12 \mathrm{H} & 0 & 2.974 & -1.053 & 4.678 & \mathrm{H} \\ \text { HETATM } & 13 \mathrm{H} & 0 & 2.352 & 0.354 & 3.785 & \mathrm{H} \\ \text { HETATM } & 14 & \mathrm{H} & 0 & 4.106 & 0.163 & 4.018 & \mathrm{H} \\ \text { HETATM } & 15 & \mathrm{C} & 0 & 4.328 & -2.236 & 2.574 & \mathrm{C} \\ \text { HETATM } & 16 \mathrm{H} & 0 & 5.007 & -2.071 & 3.399 & \mathrm{H}\end{array}$




\begin{tabular}{|c|c|c|c|c|c|c|}
\hline HETATM & $17 \mathrm{H}$ & 0 & 4.860 & -2.171 & 1.635 & $\mathrm{H}$ \\
\hline HETATM & $18 \mathrm{H}$ & 0 & 3.815 & -3.182 & 2.678 & $\mathrm{H}$ \\
\hline HETATM & $19 \mathrm{H}$ & 0 & 2.935 & -2.603 & 0.330 & $\mathrm{H}$ \\
\hline HETATM & $20 \mathrm{H}$ & 0 & 2.765 & 0.204 & -0.835 & $\mathrm{H}$ \\
\hline HETATM & $21 \mathrm{H}$ & 0 & 4.279 & -0.636 & -0.371 & $\mathrm{H}$ \\
\hline HETATM & $22 \mathrm{C}$ & 0 & 4.239 & -2.635 & -2.223 & $\mathrm{C}$ \\
\hline HETATM & $23 \mathrm{H}$ & 0 & 4.943 & -2.609 & -1.403 & $\mathrm{H}$ \\
\hline HETATM & $24 \mathrm{H}$ & 0 & 4.761 & -2.516 & -3.163 & $\mathrm{H}$ \\
\hline HETATM & $25 \mathrm{H}$ & 0 & 3.656 & -3.545 & -2.200 & $\mathrm{H}$ \\
\hline HETATM & $26 \mathrm{Cl}$ & 0 & 0.566 & -1.981 & -4.639 & $\mathrm{Cl}$ \\
\hline HETATM & $27 \mathrm{H}$ & 0 & 2.780 & -2.734 & -4.433 & $\mathrm{H}$ \\
\hline HETATM & $28 \mathrm{H}$ & 0 & 2.672 & -1.081 & -5.188 & $\mathrm{H}$ \\
\hline \multicolumn{7}{|c|}{ Conformation \#2 } \\
\hline HETATM & $1 \mathrm{C}$ & 0 & 1.753 & 0.362 & -3.516 & $\mathrm{C}$ \\
\hline HETATM & $2 \mathrm{C}$ & 0 & 1.816 & -0.269 & -2.139 & $\mathrm{C}$ \\
\hline HETATM & $3 \mathrm{H}$ & 0 & 1.054 & -0.985 & -1.958 & $\mathrm{H}$ \\
\hline HETATM & $4 \mathrm{C}$ & 0 & 2.729 & 0.018 & -1.195 & $\mathrm{C}$ \\
\hline HETATM & $5 \mathrm{C}$ & 0 & 2.703 & -0.703 & 0.145 & $\mathrm{C}$ \\
\hline HETATM & $6 \mathrm{C}$ & 0 & 3.782 & -1.797 & 0.061 & $\mathrm{C}$ \\
\hline HETATM & $7 \mathrm{H}$ & 0 & 3.562 & -2.459 & -0.765 & $\mathrm{H}$ \\
\hline HETATM & $8 \mathrm{C}$ & 0 & 3.717 & -2.534 & 1.388 & $\mathrm{C}$ \\
\hline HETATM & $9 \mathrm{H}$ & 0 & 4.222 & -2.041 & 2.180 & $\mathrm{H}$ \\
\hline HETATM & $10 \mathrm{C}$ & 0 & 3.085 & -3.701 & 1.597 & $\mathrm{C}$ \\
\hline HETATM & $11 \mathrm{C}$ & 0 & 3.094 & -4.361 & 2.962 & $\mathrm{C}$ \\
\hline HETATM & $12 \mathrm{H}$ & 0 & 2.070 & -4.414 & 3.303 & $\mathrm{H}$ \\
\hline HETATM & $13 \mathrm{H}$ & 0 & 3.693 & -3.761 & 3.633 & $\mathrm{H}$ \\
\hline HETATM & $14 \mathrm{H}$ & 0 & 3.524 & -5.346 & 2.841 & $\mathrm{H}$ \\
\hline HETATM & $15 \mathrm{C}$ & 0 & 2.336 & -4.449 & 0.507 & $\mathrm{C}$ \\
\hline HETATM & $16 \mathrm{H}$ & 0 & 3.078 & -4.904 & -0.136 & $\mathrm{H}$ \\
\hline HETATM & $17 \mathrm{H}$ & 0 & 1.725 & -3.738 & -0.030 & $\mathrm{H}$ \\
\hline HETATM & $18 \mathrm{H}$ & 0 & 1.713 & -5.201 & 0.971 & $\mathrm{H}$ \\
\hline HETATM & $19 \mathrm{H}$ & 0 & 4.758 & -1.342 & -0.036 & $\mathrm{H}$ \\
\hline HETATM & $20 \mathrm{H}$ & 0 & 1.740 & -1.174 & 0.288 & $\mathrm{H}$ \\
\hline HETATM & $21 \mathrm{H}$ & 0 & 2.938 & -0.014 & 0.945 & $\mathrm{H}$ \\
\hline HETATM & $22 \mathrm{C}$ & 0 & 3.840 & 1.034 & -1.408 & $\mathrm{C}$ \\
\hline HETATM & $23 \mathrm{H}$ & 0 & 4.300 & 1.252 & -0.454 & $\mathrm{H}$ \\
\hline HETATM & $24 \mathrm{H}$ & 0 & 3.400 & 1.931 & -1.821 & $\mathrm{H}$ \\
\hline HETATM & $25 \mathrm{H}$ & 0 & 4.555 & 0.589 & -2.086 & $\mathrm{H}$ \\
\hline HETATM & $26 \mathrm{Cl}$ & 0 & 1.560 & -1.041 & -4.607 & $\mathrm{Cl}$ \\
\hline HETATM & $27 \mathrm{H}$ & 0 & 2.678 & 0.852 & -3.783 & $\mathrm{H}$ \\
\hline HETATM & $28 \mathrm{H}$ & 0 & 0.870 & 0.978 & -3.611 & 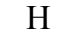 \\
\hline
\end{tabular}

Conformation \#3

$\begin{array}{llllllll}\text { HETATM } & 1 & \mathrm{C} & 0 & 4.623 & 0.585 & -2.559 & \mathrm{C} \\ \text { HETATM } & 2 & \mathrm{C} & 0 & 4.064 & -0.488 & -1.644 & \mathrm{C} \\ \text { HETATM } & 3 & \mathrm{H} & 0 & 4.797 & -1.148 & -1.254 & \mathrm{H} \\ \text { HETATM } & 4 & \mathrm{C} & 0 & 2.765 & -0.639 & -1.334 & \mathrm{C} \\ \text { HETATM } & 5 & \mathrm{C} & 0 & 2.320 & -1.749 & -0.393 & \mathrm{C} \\ \text { HETATM } & 6 & \mathrm{C} & 0 & 1.869 & -2.915 & -1.290 & \mathrm{C} \\ \text { HETATM } & 7 & \mathrm{H} & 0 & 1.051 & -2.603 & -1.922 & \mathrm{H} \\ \text { HETATM } & 8 & \mathrm{C} & 0 & 1.432 & -4.014 & -0.337 & \mathrm{C} \\ \text { HETATM } & 9 & \mathrm{H} & 0 & 2.235 & -4.576 & 0.071 & \mathrm{H}\end{array}$




$\begin{array}{llllrlll}\text { HETATM } & 10 & \mathrm{C} & 0 & 0.161 & -4.288 & 0.003 & \mathrm{C} \\ \text { HETATM } & 11 & \mathrm{C} & 0 & -0.166 & -5.421 & 0.955 & \mathrm{C} \\ \text { HETATM } & 12 & \mathrm{H} & 0 & -0.828 & -6.100 & 0.436 & \mathrm{H} \\ \text { HETATM } & 13 & \mathrm{H} & 0 & -0.642 & -4.988 & 1.824 & \mathrm{H} \\ \text { HETATM } & 14 & \mathrm{H} & 0 & 0.754 & -5.919 & 1.231 & \mathrm{H} \\ \text { HETATM } & 15 & \mathrm{C} & 0 & -1.030 & -3.515 & -0.536 & \mathrm{C} \\ \text { HETATM } & 16 & \mathrm{H} & 0 & -1.898 & -3.758 & 0.063 & \mathrm{H} \\ \text { HETATM } & 17 & \mathrm{H} & 0 & -1.171 & -3.820 & -1.563 & \mathrm{H} \\ \text { HETATM } & 18 & \mathrm{H} & 0 & -0.807 & -2.460 & -0.458 & \mathrm{H} \\ \text { HETATM } & 19 & \mathrm{H} & 0 & 2.714 & -3.268 & -1.867 & \mathrm{H} \\ \text { HETATM } & 20 & \mathrm{H} & 0 & 1.491 & -1.410 & 0.214 & \mathrm{H} \\ \text { HETATM } & 21 & \mathrm{H} & 0 & 3.157 & -2.072 & 0.212 & \mathrm{H} \\ \text { HETATM } & 22 & \mathrm{C} & 0 & 1.678 & 0.274 & -1.876 & \mathrm{C} \\ \text { HETATM } & 23 & \mathrm{H} & 0 & 0.713 & -0.164 & -1.660 & \mathrm{H} \\ \text { HETATM } & 24 & \mathrm{H} & 0 & 1.817 & 0.362 & -2.945 & \mathrm{H} \\ \text { HETATM } & 25 & \mathrm{H} & 0 & 1.784 & 1.228 & -1.378 & \mathrm{H} \\ \text { HETATM } & 26 & \mathrm{Cl} & 0 & 6.064 & 1.177 & -1.682 & \mathrm{Cl} \\ \text { HETATM } & 27 & \mathrm{H} & 0 & 3.945 & 1.420 & -2.665 & \mathrm{H} \\ \text { HETATM } & 28 & \mathrm{H} & 0 & 4.958 & 0.152 & -3.491 & \mathrm{H}\end{array}$

Conformation \#4

$\begin{array}{llllllll}\text { HETATM } & 1 & \mathrm{C} & 0 & 3.624 & -1.972 & -3.965 & \mathrm{C} \\ \text { HETATM } & 2 & \mathrm{C} & 0 & 3.095 & -1.552 & -2.607 & \mathrm{C} \\ \text { HETATM } & 3 & \mathrm{H} & 0 & 3.764 & -0.947 & -2.050 & \mathrm{H} \\ \text { HETATM } & 4 & \mathrm{C} & 0 & 1.898 & -1.898 & -2.103 & \mathrm{C} \\ \text { HETATM } & 5 & \mathrm{C} & 0 & 1.468 & -1.406 & -0.730 & \mathrm{C} \\ \text { HETATM } & 6 & \mathrm{C} & 0 & 1.812 & -2.535 & 0.258 & \mathrm{C} \\ \text { HETATM } & 7 & \mathrm{H} & 0 & 1.234 & -3.417 & 0.021 & \mathrm{H} \\ \text { HETATM } & 8 & \mathrm{C} & 0 & 1.387 & -2.025 & 1.624 & \mathrm{C} \\ \text { HETATM } & 9 & \mathrm{H} & 0 & 0.348 & -2.122 & 1.816 & \mathrm{H} \\ \text { HETATM } & 10 & \mathrm{C} & 0 & 2.207 & -1.482 & 2.539 & \mathrm{C} \\ \text { HETATM } & 11 & \mathrm{C} & 0 & 1.678 & -1.017 & 3.883 & \mathrm{C} \\ \text { HETATM } & 12 & \mathrm{H} & 0 & 1.850 & 0.048 & 3.948 & \mathrm{H} \\ \text { HETATM } & 13 & \mathrm{H} & 0 & 2.222 & -1.555 & 4.648 & \mathrm{H} \\ \text { HETATM } & 14 & \mathrm{H} & 0 & 0.622 & -1.239 & 3.936 & \mathrm{H} \\ \text { HETATM } & 15 & \mathrm{C} & 0 & 3.699 & -1.303 & 2.320 & \mathrm{C} \\ \text { HETATM } & 16 & \mathrm{H} & 0 & 4.079 & -0.626 & 3.072 & \mathrm{H} \\ \text { HETATM } & 17 & \mathrm{H} & 0 & 3.846 & -0.885 & 1.335 & \mathrm{H} \\ \text { HETATM } & 18 & \mathrm{H} & 0 & 4.155 & -2.279 & 2.413 & \mathrm{H} \\ \text { HETATM } & 19 & \mathrm{H} & 0 & 2.875 & -2.730 & 0.227 & \mathrm{H} \\ \text { HETATM } & 20 & \mathrm{H} & 0 & 0.403 & -1.218 & -0.720 & \mathrm{H} \\ \text { HETATM } & 21 & \mathrm{H} & 0 & 2.035 & -0.523 & -0.464 & \mathrm{H} \\ \text { HETATM } & 22 & \mathrm{C} & 0 & 0.910 & -2.773 & -2.858 & \mathrm{C} \\ \text { HETATM } & 23 & \mathrm{H} & 0 & 1.444 & -3.634 & -3.235 & \mathrm{H} \\ \text { HETATM } & 24 & \mathrm{H} & 0 & 0.499 & -2.173 & -3.659 & \mathrm{H} \\ \text { HETATM } & 25 & \mathrm{H} & 0 & 0.130 & -3.087 & -2.178 & \mathrm{H} \\ \text { HETATM } & 26 \mathrm{Cl} & 0 & 4.329 & -0.468 & -4.626 & \mathrm{Cl} \\ \text { HETATM } & 27 & \mathrm{H} & 0 & 2.831 & -2.277 & -4.632 & \mathrm{H} \\ \text { HETATM } & 28 & \mathrm{H} & 0 & 4.425 & -2.690 & -3.854 & \mathrm{H}\end{array}$

Conformation \#5

$\begin{array}{llllllll}\text { HETATM } & 1 \mathrm{C} & 0 & 3.146 & -1.714 & 1.564 & \mathrm{C}\end{array}$ 


$\begin{array}{llllrrrr}\text { HETATM } & 2 & \mathrm{C} & 0 & 2.468 & -0.750 & 0.610 & \mathrm{C} \\ \text { HETATM } & 3 & \mathrm{H} & 0 & 2.846 & 0.239 & 0.657 & \mathrm{H} \\ \text { HETATM } & 4 & \mathrm{C} & 0 & 1.479 & -1.071 & -0.243 & \mathrm{C} \\ \text { HETATM } & 5 & \mathrm{C} & 0 & 0.899 & -0.017 & -1.179 & \mathrm{C} \\ \text { HETATM } & 6 & \mathrm{C} & 0 & 1.783 & 0.031 & -2.445 & \mathrm{C} \\ \text { HETATM } & 7 & \mathrm{H} & 0 & 1.489 & 0.889 & -3.033 & \mathrm{H} \\ \text { HETATM } & 8 & \mathrm{C} & 0 & 1.490 & -1.216 & -3.270 & \mathrm{C} \\ \text { HETATM } & 9 & \mathrm{H} & 0 & 0.501 & -1.259 & -3.650 & \mathrm{H} \\ \text { HETATM } & 10 & \mathrm{C} & 0 & 2.368 & -2.201 & -3.527 & \mathrm{C} \\ \text { HETATM } & 11 & \mathrm{C} & 0 & 1.974 & -3.397 & -4.372 & \mathrm{C} \\ \text { HETATM } & 12 & \mathrm{H} & 0 & 2.645 & -3.429 & -5.218 & \mathrm{H} \\ \text { HETATM } & 13 & \mathrm{H} & 0 & 0.951 & -3.269 & -4.698 & \mathrm{H} \\ \text { HETATM } & 14 & \mathrm{H} & 0 & 2.073 & -4.277 & -3.751 & \mathrm{H} \\ \text { HETATM } & 15 & \mathrm{C} & 0 & 3.795 & -2.198 & -3.008 & \mathrm{C} \\ \text { HETATM } & 16 & \mathrm{H} & 0 & 3.757 & -2.054 & -1.937 & \mathrm{H} \\ \text { HETATM } & 17 & \mathrm{H} & 0 & 4.315 & -1.388 & -3.499 & \mathrm{H} \\ \text { HETATM } & 18 & \mathrm{H} & 0 & 4.253 & -3.149 & -3.243 & \mathrm{H} \\ \text { HETATM } & 19 & \mathrm{H} & 0 & 2.822 & 0.090 & -2.154 & \mathrm{H} \\ \text { HETATM } & 20 & \mathrm{H} & 0 & 0.964 & 0.946 & -0.691 & \mathrm{H} \\ \text { HETATM } & 21 & \mathrm{H} & 0 & -0.122 & -0.254 & -1.444 & \mathrm{H} \\ \text { HETATM } & 22 & \mathrm{C} & 0 & 0.916 & -2.479 & -0.348 & \mathrm{C} \\ \text { HETATM } & 23 & \mathrm{H} & 0 & 1.703 & -3.113 & -0.732 & \mathrm{H} \\ \text { HETATM } & 24 & \mathrm{H} & 0 & 0.076 & -2.474 & -1.029 & \mathrm{H} \\ \text { HETATM } & 25 & \mathrm{H} & 0 & 0.598 & -2.784 & 0.639 & \mathrm{H} \\ \text { HETATM } & 26 & \mathrm{Cl} & 0 & 4.881 & -1.522 & 1.175 & \mathrm{Cl} \\ \text { HETATM } & 27 & \mathrm{H} & 0 & 2.883 & -2.742 & 1.365 & \mathrm{H} \\ \text { HETATM } & 28 & \mathrm{H} & 0 & 3.000 & -1.401 & 2.588 & \mathrm{H}\end{array}$

Conformation \#6

$\begin{array}{llllllll}\text { HETATM } & 1 & \mathrm{C} & 0 & 3.356 & -2.977 & 1.392 & \mathrm{C} \\ \text { HETATM } & 2 & \mathrm{C} & 0 & 2.990 & -2.898 & -0.077 & \mathrm{C} \\ \text { HETATM } & 3 & \mathrm{H} & 0 & 3.422 & -3.662 & -0.674 & \mathrm{H} \\ \text { HETATM } & 4 & \mathrm{C} & 0 & 2.204 & -1.957 & -0.629 & \mathrm{C} \\ \text { HETATM } & 5 & \mathrm{C} & 0 & 1.890 & -1.990 & -2.120 & \mathrm{C} \\ \text { HETATM } & 6 & \mathrm{C} & 0 & 3.067 & -1.336 & -2.877 & \mathrm{C} \\ \text { HETATM } & 7 & \mathrm{H} & 0 & 4.002 & -1.743 & -2.518 & \mathrm{H} \\ \text { HETATM } & 8 & \mathrm{C} & 0 & 3.030 & 0.157 & -2.591 & \mathrm{C} \\ \text { HETATM } & 9 & \mathrm{H} & 0 & 3.511 & 0.429 & -1.686 & \mathrm{H} \\ \text { HETATM } & 10 & \mathrm{C} & 0 & 2.449 & 1.087 & -3.369 & \mathrm{C} \\ \text { HETATM } & 11 & \mathrm{C} & 0 & 2.490 & 2.554 & -2.990 & \mathrm{C} \\ \text { HETATM } & 12 & \mathrm{H} & 0 & 2.960 & 3.086 & -3.806 & \mathrm{H} \\ \text { HETATM } & 13 & \mathrm{H} & 0 & 3.064 & 2.662 & -2.080 & \mathrm{H} \\ \text { HETATM } & 14 & \mathrm{H} & 0 & 1.469 & 2.879 & -2.841 & \mathrm{H} \\ \text { HETATM } & 15 & \mathrm{C} & 0 & 1.737 & 0.762 & -4.670 & \mathrm{C} \\ \text { HETATM } & 16 & \mathrm{H} & 0 & 1.111 & 1.603 & -4.937 & \mathrm{H} \\ \text { HETATM } & 17 & \mathrm{H} & 0 & 1.129 & -0.117 & -4.513 & \mathrm{H} \\ \text { HETATM } & 18 & \mathrm{H} & 0 & 2.499 & 0.594 & -5.418 & \mathrm{H} \\ \text { HETATM } & 19 & \mathrm{H} & 0 & 2.941 & -1.514 & -3.935 & \mathrm{H} \\ \text { HETATM } & 20 & \mathrm{H} & 0 & 0.974 & -1.454 & -2.331 & \mathrm{H} \\ \text { HETATM } & 21 & \mathrm{H} & 0 & 1.823 & -3.023 & -2.432 & \mathrm{H} \\ \text { HETATM } & 22 & \mathrm{C} & 0 & 1.567 & -0.840 & 0.184 & \mathrm{C} \\ \text { HETATM } & 23 & \mathrm{H} & 0 & 1.202 & -0.080 & -0.493 & \mathrm{H} \\ \text { HETATM } & 24 & \mathrm{H} & 0 & 2.324 & -0.422 & 0.833 & \mathrm{H} \\ \text { HETATM } & 25 & \mathrm{H} & 0 & 0.752 & -1.277 & 0.744 & \mathrm{H}\end{array}$




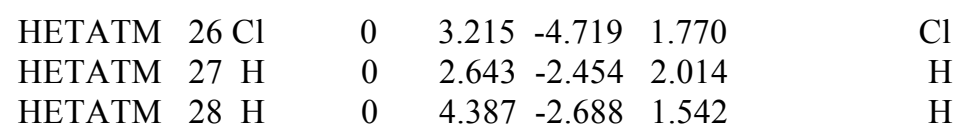

Conformation \#7

$\begin{array}{llllllll}\text { HETATM } & 1 & \mathrm{C} & 0 & 2.932 & -2.389 & -4.046 & \mathrm{C} \\ \text { HETATM } & 2 & \mathrm{C} & 0 & 3.262 & -1.391 & -2.953 & \mathrm{C} \\ \text { HETATM } & 3 & \mathrm{H} & 0 & 3.228 & -0.379 & -3.269 & \mathrm{H} \\ \text { HETATM } & 4 & \mathrm{C} & 0 & 3.583 & -1.706 & -1.686 & \mathrm{C} \\ \text { HETATM } & 5 & \mathrm{C} & 0 & 3.892 & -0.608 & -0.674 & \mathrm{C} \\ \text { HETATM } & 6 & \mathrm{C} & 0 & 2.559 & -0.142 & -0.049 & \mathrm{C} \\ \text { HETATM } & 7 & \mathrm{H} & 0 & 2.732 & 0.771 & 0.503 & \mathrm{H} \\ \text { HETATM } & 8 & \mathrm{C} & 0 & 2.080 & -1.234 & 0.894 & \mathrm{C} \\ \text { HETATM } & 9 & \mathrm{H} & 0 & 1.508 & -1.988 & 0.415 & \mathrm{H} \\ \text { HETATM } & 10 & \mathrm{C} & 0 & 2.332 & -1.294 & 2.213 & \mathrm{C} \\ \text { HETATM } & 11 & \mathrm{C} & 0 & 1.783 & -2.426 & 3.059 & \mathrm{C} \\ \text { HETATM } & 12 & \mathrm{H} & 0 & 1.180 & -3.067 & 2.431 & \mathrm{H} \\ \text { HETATM } & 13 & \mathrm{H} & 0 & 1.184 & -1.983 & 3.842 & \mathrm{H} \\ \text { HETATM } & 14 & \mathrm{H} & 0 & 2.627 & -2.968 & 3.462 & \mathrm{H} \\ \text { HETATM } & 15 & \mathrm{C} & 0 & 3.146 & -0.251 & 2.958 & \mathrm{C} \\ \text { HETATM } & 16 & \mathrm{H} & 0 & 2.494 & 0.592 & 3.139 & \mathrm{H} \\ \text { HETATM } & 17 & \mathrm{H} & 0 & 3.992 & 0.024 & 2.345 & \mathrm{H} \\ \text { HETATM } & 18 & \mathrm{H} & 0 & 3.487 & -0.684 & 3.888 & \mathrm{H} \\ \text { HETATM } & 19 & \mathrm{H} & 0 & 1.823 & 0.003 & -0.829 & \mathrm{H} \\ \text { HETATM } & 20 & \mathrm{H} & 0 & 4.324 & 0.232 & -1.201 & \mathrm{H} \\ \text { HETATM } & 21 & \mathrm{H} & 0 & 4.556 & -0.971 & 0.098 & \mathrm{H} \\ \text { HETATM } & 22 & \mathrm{C} & 0 & 3.649 & -3.144 & -1.195 & \mathrm{C} \\ \text { HETATM } & 23 & \mathrm{H} & 0 & 3.978 & -3.150 & -0.165 & \mathrm{H} \\ \text { HETATM } & 24 & \mathrm{H} & 0 & 4.355 & -3.673 & -1.820 & \mathrm{H} \\ \text { HETATM } & 25 & \mathrm{H} & 0 & 2.653 & -3.559 & -1.273 & \mathrm{H} \\ \text { HETATM } & 26 \mathrm{Cl} & 0 & 1.419 & -1.749 & -4.750 & \mathrm{Cl} \\ \text { HETATM } & 27 & \mathrm{H} & 0 & 2.715 & -3.371 & -3.651 & \mathrm{H} \\ \text { HETATM } & 28 & \mathrm{H} & 0 & 3.689 & -2.371 & -4.817 & \mathrm{H} \\ & & & & & & & \end{array}$

Conformation \#8

$\begin{array}{llllllll}\text { HETATM } & 1 & \mathrm{C} & 0 & 4.320 & -2.705 & 0.846 & \mathrm{C} \\ \text { HETATM } & 2 & \mathrm{C} & 0 & 3.712 & -2.608 & -0.539 & \mathrm{C} \\ \text { HETATM } & 3 & \mathrm{H} & 0 & 2.792 & -3.125 & -0.642 & \mathrm{H} \\ \text { HETATM } & 4 & \mathrm{C} & 0 & 4.240 & -1.935 & -1.577 & \mathrm{C} \\ \text { HETATM } & 5 & \mathrm{C} & 0 & 3.514 & -1.892 & -2.917 & \mathrm{C} \\ \text { HETATM } & 6 & \mathrm{C} & 0 & 2.821 & -0.518 & -3.048 & \mathrm{C} \\ \text { HETATM } & 7 & \mathrm{H} & 0 & 3.512 & 0.274 & -2.797 & \mathrm{H} \\ \text { HETATM } & 8 & \mathrm{C} & 0 & 1.669 & -0.506 & -2.055 & \mathrm{C} \\ \text { HETATM } & 9 & \mathrm{H} & 0 & 1.974 & -0.359 & -1.048 & \mathrm{H} \\ \text { HETATM } & 10 & \mathrm{C} & 0 & 0.371 & -0.679 & -2.359 & \mathrm{C} \\ \text { HETATM } & 11 & \mathrm{C} & 0 & -0.690 & -0.647 & -1.276 & \mathrm{C} \\ \text { HETATM } & 12 & \mathrm{H} & 0 & -1.176 & -1.612 & -1.270 & \mathrm{H} \\ \text { HETATM } & 13 & \mathrm{H} & 0 & -1.381 & 0.147 & -1.523 & \mathrm{H} \\ \text { HETATM } & 14 & \mathrm{H} & 0 & -0.209 & -0.456 & -0.326 & \mathrm{H} \\ \text { HETATM } & 15 & \mathrm{C} & 0 & -0.130 & -0.910 & -3.773 & \mathrm{C} \\ \text { HETATM } & 16 & \mathrm{H} & 0 & -0.009 & 0.019 & -4.312 & \mathrm{H} \\ \text { HETATM } & 17 & \mathrm{H} & 0 & -1.172 & -1.197 & -3.727 & \mathrm{H}\end{array}$




$\begin{array}{llllllll}\text { HETATM } & 18 & \mathrm{H} & 0 & 0.460 & -1.705 & -4.206 & \mathrm{H} \\ \text { HETATM } & 19 & \mathrm{H} & 0 & 2.451 & -0.414 & -4.057 & \mathrm{H} \\ \text { HETATM } & 20 & \mathrm{H} & 0 & 2.761 & -2.669 & -2.958 & \mathrm{H} \\ \text { HETATM } & 21 & \mathrm{H} & 0 & 4.236 & -2.000 & -3.715 & \mathrm{H} \\ \text { HETATM } & 22 & \mathrm{C} & 0 & 5.550 & -1.169 & -1.476 & \mathrm{C} \\ \text { HETATM } & 23 & \mathrm{H} & 0 & 5.369 & -0.307 & -0.849 & \mathrm{H} \\ \text { HETATM } & 24 & \mathrm{H} & 0 & 5.853 & -0.865 & -2.468 & \mathrm{H} \\ \text { HETATM } & 25 & \mathrm{H} & 0 & 6.289 & -1.830 & -1.046 & \mathrm{H} \\ \text { HETATM } & 26 & \mathrm{Cl} & 0 & 2.977 & -2.226 & 1.924 & \mathrm{Cl} \\ \text { HETATM } & 27 & \mathrm{H} & 0 & 5.120 & -1.994 & 0.993 & \mathrm{H} \\ \text { HETATM } & 28 & \mathrm{H} & 0 & 4.579 & -3.730 & 1.075 & \mathrm{H}\end{array}$

Conformation \#9

$\begin{array}{llllllll}\text { HETATM } & 1 & \mathrm{C} & 0 & 3.648 & -3.527 & -0.076 & \mathrm{C} \\ \text { HETATM } & 2 & \mathrm{C} & 0 & 2.476 & -2.566 & -0.042 & \mathrm{C} \\ \text { HETATM } & 3 & \mathrm{H} & 0 & 1.533 & -3.034 & 0.085 & \mathrm{H} \\ \text { HETATM } & 4 & \mathrm{C} & 0 & 2.570 & -1.231 & -0.176 & \mathrm{C} \\ \text { HETATM } & 5 & \mathrm{C} & 0 & 1.320 & -0.361 & -0.128 & \mathrm{C} \\ \text { HETATM } & 6 & \mathrm{C} & 0 & 0.731 & -0.290 & -1.555 & \mathrm{C} \\ \text { HETATM } & 7 & \mathrm{H} & 0 & 0.693 & -1.282 & -1.979 & \mathrm{H} \\ \text { HETATM } & 8 & \mathrm{C} & 0 & 1.603 & 0.651 & -2.376 & \mathrm{C} \\ \text { HETATM } & 9 & \mathrm{H} & 0 & 1.586 & 1.657 & -2.041 & \mathrm{H} \\ \text { HETATM } & 10 & \mathrm{C} & 0 & 2.347 & 0.296 & -3.438 & \mathrm{C} \\ \text { HETATM } & 11 & \mathrm{C} & 0 & 3.173 & 1.322 & -4.190 & \mathrm{C} \\ \text { HETATM } & 12 & \mathrm{H} & 0 & 4.208 & 1.020 & -4.115 & \mathrm{H} \\ \text { HETATM } & 13 & \mathrm{H} & 0 & 3.022 & 2.291 & -3.734 & \mathrm{H} \\ \text { HETATM } & 14 & \mathrm{H} & 0 & 2.832 & 1.323 & -5.216 & \mathrm{H} \\ \text { HETATM } & 15 & \mathrm{C} & 0 & 2.417 & -1.125 & -3.965 & \mathrm{C} \\ \text { HETATM } & 16 & \mathrm{H} & 0 & 3.165 & -1.172 & -4.744 & \mathrm{H} \\ \text { HETATM } & 17 & \mathrm{H} & 0 & 1.439 & -1.371 & -4.356 & \mathrm{H} \\ \text { HETATM } & 18 & \mathrm{H} & 0 & 2.689 & -1.767 & -3.139 & \mathrm{H} \\ \text { HETATM } & 19 & \mathrm{H} & 0 & -0.254 & 0.150 & -1.493 & \mathrm{H} \\ \text { HETATM } & 20 & \mathrm{H} & 0 & 1.556 & 0.631 & 0.231 & \mathrm{H} \\ \text { HETATM } & 21 & \mathrm{H} & 0 & 0.592 & -0.846 & 0.508 & \mathrm{H} \\ \text { HETATM } & 22 & \mathrm{C} & 0 & 3.901 & -0.524 & -0.374 & \mathrm{C} \\ \text { HETATM } & 23 & \mathrm{H} & 0 & 4.466 & -0.634 & 0.541 & \mathrm{H} \\ \text { HETATM } & 24 & \mathrm{H} & 0 & 3.717 & 0.521 & -0.584 & \mathrm{H} \\ \text { HETATM } & 25 & \mathrm{H} & 0 & 4.399 & -0.987 & -1.215 & \mathrm{H} \\ \text { HETATM } & 26 & \mathrm{Cl} & 0 & 3.311 & -4.648 & 1.275 & \mathrm{Cl} \\ \text { HETATM } & 27 & \mathrm{H} & 0 & 4.584 & -3.033 & 0.139 & \mathrm{H} \\ \text { HETATM } & 28 & \mathrm{H} & 0 & 3.640 & -4.101 & -0.992 & \mathrm{H}\end{array}$

Conformation \#10

$\begin{array}{llllrrrr}\text { HETATM } & 1 & \mathrm{C} & 0 & 5.179 & -2.046 & -0.951 & \mathrm{C} \\ \text { HETATM } & 2 & \mathrm{C} & 0 & 3.668 & -2.162 & -0.995 & \mathrm{C} \\ \text { HETATM } & 3 & \mathrm{H} & 0 & 3.261 & -2.116 & -1.974 & \mathrm{H} \\ \text { HETATM } & 4 & \mathrm{C} & 0 & 2.866 & -2.294 & 0.076 & \mathrm{C} \\ \text { HETATM } & 5 & \mathrm{C} & 0 & 1.355 & -2.399 & -0.100 & \mathrm{C} \\ \text { HETATM } & 6 & \mathrm{C} & 0 & 0.726 & -1.036 & 0.261 & \mathrm{C} \\ \text { HETATM } & 7 & \mathrm{H} & 0 & -0.347 & -1.151 & 0.303 & \mathrm{H} \\ \text { HETATM } & 8 & \mathrm{C} & 0 & 1.111 & -0.059 & -0.840 & \mathrm{C} \\ \text { HETATM } & 9 & \mathrm{H} & 0 & 2.099 & 0.316 & -0.752 & \mathrm{H}\end{array}$




$\begin{array}{llllrrrr}\text { HETATM } & 10 & \mathrm{C} & 0 & 0.321 & 0.318 & -1.861 & \mathrm{C} \\ \text { HETATM } & 11 & \mathrm{C} & 0 & 0.811 & 1.307 & -2.900 & \mathrm{C} \\ \text { HETATM } & 12 & \mathrm{H} & 0 & 0.814 & 0.800 & -3.854 & \mathrm{H} \\ \text { HETATM } & 13 & \mathrm{H} & 0 & 1.809 & 1.625 & -2.632 & \mathrm{H} \\ \text { HETATM } & 14 & \mathrm{H} & 0 & 0.128 & 2.145 & -2.896 & \mathrm{H} \\ \text { HETATM } & 15 & \mathrm{C} & 0 & -1.095 & -0.195 & -2.053 & \mathrm{C} \\ \text { HETATM } & 16 & \mathrm{H} & 0 & -1.075 & -1.271 & -1.952 & \mathrm{H} \\ \text { HETATM } & 17 & \mathrm{H} & 0 & -1.430 & 0.081 & -3.043 & \mathrm{H} \\ \text { HETATM } & 18 & \mathrm{H} & 0 & -1.709 & 0.268 & -1.293 & \mathrm{H} \\ \text { HETATM } & 19 & \mathrm{H} & 0 & 1.121 & -0.680 & 1.202 & \mathrm{H} \\ \text { HETATM } & 20 & \mathrm{H} & 0 & 0.975 & -3.153 & 0.576 & \mathrm{H} \\ \text { HETATM } & 21 & \mathrm{H} & 0 & 1.116 & -2.635 & -1.129 & \mathrm{H} \\ \text { HETATM } & 22 & \mathrm{C} & 0 & 3.403 & -2.356 & 1.496 & \mathrm{C} \\ \text { HETATM } & 23 & \mathrm{H} & 0 & 3.967 & -1.450 & 1.673 & \mathrm{H} \\ \text { HETATM } & 24 & \mathrm{H} & 0 & 4.026 & -3.237 & 1.568 & \mathrm{H} \\ \text { HETATM } & 25 & \mathrm{H} & 0 & 2.572 & -2.425 & 2.184 & \mathrm{H} \\ \text { HETATM } & 26 & \mathrm{Cl} & 0 & 5.728 & -3.280 & -2.121 & \mathrm{Cl} \\ \text { HETATM } & 27 & \mathrm{H} & 0 & 5.582 & -2.312 & 0.014 & \mathrm{H} \\ \text { HETATM } & 28 & \mathrm{H} & 0 & 5.493 & -1.079 & -1.319 & \mathrm{H}\end{array}$

Conformation \#11

$\begin{array}{llllllll}\text { HETATM } & 1 & \mathrm{C} & 0 & 4.132 & 0.019 & -0.900 & \mathrm{C} \\ \text { HETATM } & 2 & \mathrm{C} & 0 & 2.752 & -0.512 & -1.233 & \mathrm{C} \\ \text { HETATM } & 3 & \mathrm{H} & 0 & 2.035 & -0.360 & -0.466 & \mathrm{H} \\ \text { HETATM } & 4 & \mathrm{C} & 0 & 2.419 & -1.153 & -2.366 & \mathrm{C} \\ \text { HETATM } & 5 & \mathrm{C} & 0 & 0.994 & -1.646 & -2.582 & \mathrm{C} \\ \text { HETATM } & 6 & \mathrm{C} & 0 & 0.945 & -3.146 & -2.213 & \mathrm{C} \\ \text { HETATM } & 7 & \mathrm{H} & 0 & -0.014 & -3.540 & -2.520 & \mathrm{H} \\ \text { HETATM } & 8 & \mathrm{C} & 0 & 1.021 & -3.247 & -0.695 & \mathrm{C} \\ \text { HETATM } & 9 & \mathrm{H} & 0 & 0.198 & -2.804 & -0.193 & \mathrm{H} \\ \text { HETATM } & 10 & \mathrm{C} & 0 & 2.013 & -3.828 & 0.001 & \mathrm{C} \\ \text { HETATM } & 11 & \mathrm{C} & 0 & 1.983 & -3.868 & 1.516 & \mathrm{C} \\ \text { HETATM } & 12 & \mathrm{H} & 0 & 2.007 & -4.907 & 1.813 & \mathrm{H} \\ \text { HETATM } & 13 & \mathrm{H} & 0 & 1.075 & -3.392 & 1.858 & \mathrm{H} \\ \text { HETATM } & 14 & \mathrm{H} & 0 & 2.853 & -3.332 & 1.870 & \mathrm{H} \\ \text { HETATM } & 15 & \mathrm{C} & 0 & 3.220 & -4.471 & -0.656 & \mathrm{C} \\ \text { HETATM } & 16 & \mathrm{H} & 0 & 2.861 & -5.296 & -1.256 & \mathrm{H} \\ \text { HETATM } & 17 & \mathrm{H} & 0 & 3.891 & -4.821 & 0.116 & \mathrm{H} \\ \text { HETATM } & 18 & \mathrm{H} & 0 & 3.699 & -3.714 & -1.262 & \mathrm{H} \\ \text { HETATM } & 19 & \mathrm{H} & 0 & 1.759 & -3.668 & -2.693 & \mathrm{H} \\ \text { HETATM } & 20 & \mathrm{H} & 0 & 0.738 & -1.533 & -3.627 & \mathrm{H} \\ \text { HETATM } & 21 & \mathrm{H} & 0 & 0.311 & -1.096 & -1.948 & \mathrm{H} \\ \text { HETATM } & 22 & \mathrm{C} & 0 & 3.415 & -1.414 & -3.485 & \mathrm{C} \\ \text { HETATM } & 23 & \mathrm{H} & 0 & 3.616 & -0.464 & -3.961 & \mathrm{H} \\ \text { HETATM } & 24 & \mathrm{H} & 0 & 2.977 & -2.109 & -4.187 & \mathrm{H} \\ \text { HETATM } & 25 & \mathrm{H} & 0 & 4.306 & -1.839 & -3.045 & \mathrm{H} \\ \text { HETATM } & 26 & \mathrm{Cl} & 0 & 3.813 & 1.667 & -0.285 & \mathrm{Cl} \\ \text { HETATM } & 27 & \mathrm{H} & 0 & 4.752 & 0.117 & -1.779 & \mathrm{H} \\ \text { HETATM } & 28 & \mathrm{H} & 0 & 4.572 & -0.555 & -0.097 & \mathrm{H}\end{array}$

Conformation \#12

$\begin{array}{llllllll}\text { HETATM } & 1 \mathrm{C} & 0 & 1.100 & -2.981 & 0.176 & \mathrm{C}\end{array}$ 


$\begin{array}{llllllll}\text { HETATM } & 2 & \mathrm{C} & 0 & 1.165 & -1.843 & -0.823 & \mathrm{C} \\ \text { HETATM } & 3 & \mathrm{H} & 0 & 1.106 & -0.880 & -0.383 & \mathrm{H} \\ \text { HETATM } & 4 & \mathrm{C} & 0 & 1.296 & -1.982 & -2.154 & \mathrm{C} \\ \text { HETATM } & 5 & \mathrm{C} & 0 & 1.358 & -0.755 & -3.057 & \mathrm{C} \\ \text { HETATM } & 6 & \mathrm{C} & 0 & 2.846 & -0.439 & -3.330 & \mathrm{C} \\ \text { HETATM } & 7 & \mathrm{H} & 0 & 3.382 & -1.350 & -3.552 & \mathrm{H} \\ \text { HETATM } & 8 & \mathrm{C} & 0 & 3.394 & 0.259 & -2.093 & \mathrm{C} \\ \text { HETATM } & 9 & \mathrm{H} & 0 & 2.974 & 1.216 & -1.917 & \mathrm{H} \\ \text { HETATM } & 10 & \mathrm{C} & 0 & 4.316 & -0.246 & -1.255 & \mathrm{C} \\ \text { HETATM } & 11 & \mathrm{C} & 0 & 4.774 & 0.531 & -0.037 & \mathrm{C} \\ \text { HETATM } & 12 & \mathrm{H} & 0 & 4.273 & 1.489 & -0.028 & \mathrm{H} \\ \text { HETATM } & 13 & \mathrm{H} & 0 & 5.845 & 0.657 & -0.116 & \mathrm{H} \\ \text { HETATM } & 14 & \mathrm{H} & 0 & 4.503 & -0.051 & 0.833 & \mathrm{H} \\ \text { HETATM } & 15 & \mathrm{C} & 0 & 4.943 & -1.616 & -1.437 & \mathrm{C} \\ \text { HETATM } & 16 & \mathrm{H} & 0 & 5.480 & -1.606 & -2.375 & \mathrm{H} \\ \text { HETATM } & 17 & \mathrm{H} & 0 & 4.140 & -2.338 & -1.440 & \mathrm{H} \\ \text { HETATM } & 18 & \mathrm{H} & 0 & 5.614 & -1.803 & -0.611 & \mathrm{H} \\ \text { HETATM } & 19 & \mathrm{H} & 0 & 2.900 & 0.264 & -4.150 & \mathrm{H} \\ \text { HETATM } & 20 & \mathrm{H} & 0 & 0.892 & 0.090 & -2.568 & \mathrm{H} \\ \text { HETATM } & 21 & \mathrm{H} & 0 & 0.871 & -0.982 & -3.995 & \mathrm{H} \\ \text { HETATM } & 22 & \mathrm{C} & 0 & 1.408 & -3.340 & -2.828 & \mathrm{C} \\ \text { HETATM } & 23 & \mathrm{H} & 0 & 1.483 & -3.198 & -3.897 & \mathrm{H} \\ \text { HETATM } & 24 & \mathrm{H} & 0 & 0.517 & -3.901 & -2.582 & \mathrm{H} \\ \text { HETATM } & 25 & \mathrm{H} & 0 & 2.300 & -3.817 & -2.448 & \mathrm{H} \\ \text { HETATM } & 26 & \mathrm{Cl} & 0 & 2.383 & -2.582 & 1.356 & \mathrm{Cl} \\ \text { HETATM } & 27 & \mathrm{H} & 0 & 1.355 & -3.932 & -0.268 & \mathrm{H} \\ \text { HETATM } & 28 & \mathrm{H} & 0 & 0.155 & -2.969 & 0.700 & \mathrm{H}\end{array}$

Conformation \#13

$\begin{array}{llllllll}\text { HETATM } & 1 & \mathrm{C} & 0 & 2.299 & -1.234 & -3.737 & \mathrm{C} \\ \text { HETATM } & 2 & \mathrm{C} & 0 & 2.362 & -0.438 & -2.449 & \mathrm{C} \\ \text { HETATM } & 3 & \mathrm{H} & 0 & 1.433 & -0.022 & -2.150 & \mathrm{H} \\ \text { HETATM } & 4 & \mathrm{C} & 0 & 3.471 & -0.246 & -1.712 & \mathrm{C} \\ \text { HETATM } & 5 & \mathrm{C} & 0 & 3.403 & 0.542 & -0.408 & \mathrm{C} \\ \text { HETATM } & 6 & \mathrm{C} & 0 & 3.672 & -0.421 & 0.774 & \mathrm{C} \\ \text { HETATM } & 7 & \mathrm{H} & 0 & 4.640 & -0.884 & 0.646 & \mathrm{H} \\ \text { HETATM } & 8 & \mathrm{C} & 0 & 2.585 & -1.486 & 0.786 & \mathrm{C} \\ \text { HETATM } & 9 & \mathrm{H} & 0 & 1.684 & -1.214 & 0.296 & \mathrm{H} \\ \text { HETATM } & 10 & \mathrm{C} & 0 & 2.709 & -2.692 & 1.365 & \mathrm{C} \\ \text { HETATM } & 11 & \mathrm{C} & 0 & 1.584 & -3.706 & 1.298 & \mathrm{C} \\ \text { HETATM } & 12 & \mathrm{H} & 0 & 0.789 & -3.301 & 0.688 & \mathrm{H} \\ \text { HETATM } & 13 & \mathrm{H} & 0 & 1.244 & -3.881 & 2.309 & \mathrm{H} \\ \text { HETATM } & 14 & \mathrm{H} & 0 & 1.986 & -4.606 & 0.852 & \mathrm{H} \\ \text { HETATM } & 15 & \mathrm{C} & 0 & 3.962 & -3.145 & 2.093 & \mathrm{C} \\ \text { HETATM } & 16 & \mathrm{H} & 0 & 4.405 & -2.291 & 2.584 & \mathrm{H} \\ \text { HETATM } & 17 & \mathrm{H} & 0 & 4.625 & -3.566 & 1.350 & \mathrm{H} \\ \text { HETATM } & 18 & \mathrm{H} & 0 & 3.676 & -3.886 & 2.827 & \mathrm{H} \\ \text { HETATM } & 19 & \mathrm{H} & 0 & 3.605 & 0.142 & 1.694 & \mathrm{H} \\ \text { HETATM } & 20 & \mathrm{H} & 0 & 2.420 & 0.976 & -0.287 & \mathrm{H} \\ \text { HETATM } & 21 & \mathrm{H} & 0 & 4.171 & 1.304 & -0.417 & \mathrm{H} \\ \text { HETATM } & 22 & \mathrm{C} & 0 & 4.827 & -0.810 & -2.106 & \mathrm{C} \\ \text { HETATM } & 23 & \mathrm{H} & 0 & 4.855 & -1.836 & -1.765 & \mathrm{H} \\ \text { HETATM } & 24 & \mathrm{H} & 0 & 5.594 & -0.220 & -1.624 & \mathrm{H} \\ \text { HETATM } & 25 & \mathrm{H} & 0 & 4.933 & -0.743 & -3.179 & \mathrm{H}\end{array}$




\begin{tabular}{|c|c|c|c|c|c|c|}
\hline HETATM & $26 \mathrm{Cl}$ & 0 & 0.720 & -2.068 & -3.648 & $\mathrm{Cl}$ \\
\hline HETATM & $27 \mathrm{H}$ & 0 & 3.063 & -1.997 & -3.772 & $\mathrm{H}$ \\
\hline HETATM & $28 \mathrm{H}$ & 0 & 2.275 & -0.572 & -4.592 & $\mathrm{H}$ \\
\hline \multicolumn{7}{|c|}{ Conformation \#14 } \\
\hline HETATM & $1 \mathrm{C}$ & 0 & 3.655 & -1.007 & -3.470 & $\mathrm{C}$ \\
\hline HETATM & $2 \mathrm{C}$ & 0 & 3.231 & -0.984 & -2.014 & $\mathrm{C}$ \\
\hline HETATM & $3 \mathrm{H}$ & 0 & 3.451 & -1.888 & -1.509 & $\mathrm{H}$ \\
\hline HETATM & $4 \mathrm{C}$ & 0 & 2.624 & 0.050 & -1.396 & $\mathrm{C}$ \\
\hline HETATM & $5 \mathrm{C}$ & 0 & 2.213 & 0.067 & 0.083 & $\mathrm{C}$ \\
\hline HETATM & $6 \mathrm{C}$ & 0 & 2.323 & -1.310 & 0.782 & $\mathrm{C}$ \\
\hline HETATM & $7 \mathrm{H}$ & 0 & 3.307 & -1.733 & 0.638 & $\mathrm{H}$ \\
\hline HETATM & $8 \mathrm{C}$ & 0 & 1.253 & -2.227 & 0.206 & $\mathrm{C}$ \\
\hline HETATM & $9 \mathrm{H}$ & 0 & 0.905 & -1.961 & -0.761 & $\mathrm{H}$ \\
\hline HETATM & $10 \mathrm{C}$ & 0 & 0.765 & -3.311 & 0.831 & $\mathrm{C}$ \\
\hline HETATM & $11 \mathrm{C}$ & 0 & -0.317 & -4.160 & 0.194 & $\mathrm{C}$ \\
\hline HETATM & $12 \mathrm{H}$ & 0 & -1.151 & -4.192 & 0.882 & $\mathrm{H}$ \\
\hline HETATM & $13 \mathrm{H}$ & 0 & 0.098 & -5.144 & 0.027 & $\mathrm{H}$ \\
\hline HETATM & $14 \mathrm{H}$ & 0 & -0.610 & -3.703 & -0.741 & $\mathrm{H}$ \\
\hline HETATM & $15 \mathrm{C}$ & 0 & 1.227 & -3.762 & 2.206 & $\mathrm{C}$ \\
\hline HETATM & $16 \mathrm{H}$ & 0 & 2.273 & -3.515 & 2.320 & $\mathrm{H}$ \\
\hline HETATM & $17 \mathrm{H}$ & 0 & 1.093 & -4.833 & 2.271 & $\mathrm{H}$ \\
\hline HETATM & $18 \mathrm{H}$ & 0 & 0.615 & -3.247 & 2.933 & $\mathrm{H}$ \\
\hline HETATM & $19 \mathrm{H}$ & 0 & 2.110 & -1.154 & 1.830 & $\mathrm{H}$ \\
\hline HETATM & $20 \mathrm{H}$ & 0 & 1.183 & 0.391 & 0.158 & $\mathrm{H}$ \\
\hline HETATM & $21 \mathrm{H}$ & 0 & 2.879 & 0.746 & 0.597 & $\mathrm{H}$ \\
\hline HETATM & $22 \mathrm{C}$ & 0 & 2.316 & 1.346 & -2.129 & $\mathrm{C}$ \\
\hline HETATM & $23 \mathrm{H}$ & 0 & 3.262 & 1.791 & -2.407 & $\mathrm{H}$ \\
\hline HETATM & $24 \mathrm{H}$ & 0 & 1.770 & 2.006 & -1.469 & $\mathrm{H}$ \\
\hline HETATM & $25 \mathrm{H}$ & 0 & 1.717 & 1.101 & -2.994 & $\mathrm{H}$ \\
\hline HETATM & $26 \mathrm{Cl}$ & 0 & 5.374 & -1.490 & -3.395 & $\mathrm{Cl}$ \\
\hline HETATM & $27 \mathrm{H}$ & 0 & 3.615 & -0.030 & -3.928 & $\mathrm{H}$ \\
\hline HETATM & $28 \mathrm{H}$ & 0 & 3.119 & -1.777 & -4.007 & 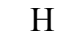 \\
\hline
\end{tabular}




\subsection{Refined Reactant Geometries}

Prenyl Chloride

$\begin{array}{llllrrrr}\text { HETATM } & 1 & \mathrm{C} & 0 & 0.231 & -0.630 & 0.454 & \mathrm{C} \\ \text { HETATM } & 2 & \mathrm{H} & 0 & 0.194 & -1.716 & 0.494 & \mathrm{H} \\ \text { HETATM } & 3 & \mathrm{C} & 0 & 1.367 & -0.043 & 0.046 & \mathrm{C} \\ \text { HETATM } & 4 & \mathrm{C} & 0 & 1.573 & 1.442 & -0.091 & \mathrm{C} \\ \text { HETATM } & 5 & \mathrm{H} & 0 & 2.371 & 1.781 & 0.579 & \mathrm{H} \\ \text { HETATM } & 6 & \mathrm{H} & 0 & 1.902 & 1.683 & -1.107 & \mathrm{H} \\ \text { HETATM } & 7 & \mathrm{H} & 0 & 0.683 & 2.034 & 0.119 & \mathrm{H} \\ \text { HETATM } & 8 & \mathrm{C} & 0 & 2.568 & -0.878 & -0.317 & \mathrm{C} \\ \text { HETATM } & 9 & \mathrm{H} & 0 & 2.864 & -0.699 & -1.357 & \mathrm{H} \\ \text { HETATM } & 10 & \mathrm{H} & 0 & 3.432 & -0.608 & 0.301 & \mathrm{H} \\ \text { HETATM } & 11 & \mathrm{H} & 0 & 2.380 & -1.946 & -0.194 & \mathrm{H} \\ \text { HETATM } & 12 & \mathrm{C} & 0 & -1.012 & 0.054 & 0.878 & \mathrm{C} \\ \text { HETATM } & 13 \mathrm{H} & 0 & -1.476 & -0.422 & 1.738 & \mathrm{H} \\ \text { HETATM } & 14 \mathrm{H} & 0 & -0.904 & 1.117 & 1.064 & \mathrm{H} \\ \text { HETATM } & 15 \mathrm{Cl} & 0 & -2.342 & -0.053 & -0.439 & \mathrm{Cl}\end{array}$

Geranyl Chloride Far Up Conformation

\begin{tabular}{|c|c|c|c|c|c|c|}
\hline HETATM & $1 \mathrm{C}$ & 0 & -2.989 & -0.111 & 0.011 & $\mathrm{C}$ \\
\hline HETATM & $2 \mathrm{C}$ & 0 & -1.817 & -0.756 & -0.626 & $\mathrm{C}$ \\
\hline HETATM & $3 \mathrm{H}$ & 0 & -1.791 & -0.686 & -1.711 & $\mathrm{H}$ \\
\hline HETATM & $4 \mathrm{C}$ & 0 & -0.823 & -1.411 & -0.004 & $\mathrm{C}$ \\
\hline HETATM & $5 \mathrm{C}$ & 0 & 0.267 & -2.074 & -0.818 & $\mathrm{C}$ \\
\hline HETATM & $6 \mathrm{C}$ & 0 & 1.705 & -1.590 & -0.513 & $\mathrm{C}$ \\
\hline HETATM & $7 \mathrm{H}$ & 0 & 1.969 & -1.829 & 0.519 & $\mathrm{H}$ \\
\hline HETATM & $8 \mathrm{C}$ & 0 & 1.917 & -0.131 & -0.817 & $\mathrm{C}$ \\
\hline HETATM & $9 \mathrm{H}$ & 0 & 1.546 & 0.179 & -1.795 & $\mathrm{H}$ \\
\hline HETATM & $10 \mathrm{C}$ & 0 & 2.501 & 0.807 & -0.061 & $\mathrm{C}$ \\
\hline HETATM & $11 \mathrm{C}$ & 0 & 2.632 & 2.228 & -0.555 & $\mathrm{C}$ \\
\hline HETATM & $12 \mathrm{H}$ & 0 & 2.203 & 2.357 & -1.551 & $\mathrm{H}$ \\
\hline HETATM & $13 \mathrm{H}$ & 0 & 3.684 & 2.536 & -0.596 & $\mathrm{H}$ \\
\hline HETATM & $14 \mathrm{H}$ & 0 & 2.132 & 2.929 & 0.123 & $\mathrm{H}$ \\
\hline HETATM & $15 \mathrm{C}$ & 0 & 3.086 & 0.575 & 1.310 & $\mathrm{C}$ \\
\hline HETATM & $16 \mathrm{H}$ & 0 & 2.964 & -0.447 & 1.670 & $\mathrm{H}$ \\
\hline HETATM & $17 \mathrm{H}$ & 0 & 2.629 & 1.247 & 2.045 & $\mathrm{H}$ \\
\hline HETATM & $18 \mathrm{H}$ & 0 & 4.159 & 0.800 & 1.313 & $\mathrm{H}$ \\
\hline HETATM & $19 \mathrm{H}$ & 0 & 2.380 & -2.191 & -1.138 & $\mathrm{H}$ \\
\hline HETATM & $20 \mathrm{H}$ & 0 & 0.062 & -1.934 & -1.883 & $\mathrm{H}$ \\
\hline HETATM & $21 \mathrm{H}$ & 0 & 0.231 & -3.155 & -0.633 & $\mathrm{H}$ \\
\hline HETATM & $22 \mathrm{C}$ & 0 & -0.703 & -1.565 & 1.489 & $\mathrm{C}$ \\
\hline HETATM & $23 \mathrm{H}$ & 0 & 0.163 & -1.009 & 1.861 & $\mathrm{H}$ \\
\hline HETATM & $24 \mathrm{H}$ & 0 & -0.540 & -2.614 & 1.756 & $\mathrm{H}$ \\
\hline HETATM & $25 \mathrm{H}$ & 0 & -1.575 & -1.211 & 2.038 & $\mathrm{H}$ \\
\hline HETATM & $26 \mathrm{Cl}$ & 0 & -2.812 & 1.755 & 0.058 & $\mathrm{Cl}$ \\
\hline HETATM & $27 \mathrm{H}$ & 0 & -3.154 & -0.388 & 1.046 & $\mathrm{H}$ \\
\hline HETATM & $28 \mathrm{H}$ & 0 & -3.903 & -0.257 & -0.558 & $\mathrm{H}$ \\
\hline
\end{tabular}

Geranyl Chloride Up Conformation

$\begin{array}{llllllll}\text { HETATM } & 1 & \mathrm{C} & 0 & 3.028 & 0.116 & 0.624 & \mathrm{C}\end{array}$ 


$\begin{array}{llllrrrr}\text { HETATM } & 2 & \mathrm{H} & 0 & 3.586 & 0.302 & 1.537 & \mathrm{H} \\ \text { HETATM } & 3 & \mathrm{H} & 0 & 3.629 & -0.477 & -0.057 & \mathrm{H} \\ \text { HETATM } & 4 & \mathrm{Cl} & 0 & 2.954 & 1.807 & -0.182 & \mathrm{Cl} \\ \text { HETATM } & 5 & \mathrm{C} & 0 & 1.675 & -0.425 & 0.889 & \mathrm{C} \\ \text { HETATM } & 6 & \mathrm{H} & 0 & 1.179 & -0.012 & 1.764 & \mathrm{H} \\ \text { HETATM } & 7 & \mathrm{C} & 0 & 1.027 & -1.359 & 0.172 & \mathrm{C} \\ \text { HETATM } & 8 & \mathrm{C} & 0 & -0.336 & -1.841 & 0.619 & \mathrm{C} \\ \text { HETATM } & 9 & \mathrm{H} & 0 & -0.303 & -2.934 & 0.717 & \mathrm{H} \\ \text { HETATM } & 10 & \mathrm{H} & 0 & -0.561 & -1.440 & 1.611 & \mathrm{H} \\ \text { HETATM } & 11 & \mathrm{C} & 0 & 1.567 & -2.005 & -1.077 & \mathrm{C} \\ \text { HETATM } & 12 & \mathrm{H} & 0 & 1.572 & -3.095 & -0.973 & \mathrm{H} \\ \text { HETATM } & 13 & \mathrm{H} & 0 & 0.927 & -1.780 & -1.936 & \mathrm{H} \\ \text { HETATM } & 14 & \mathrm{H} & 0 & 2.577 & -1.687 & -1.335 & \mathrm{H} \\ \text { HETATM } & 15 & \mathrm{C} & 0 & -1.509 & -1.489 & -0.336 & \mathrm{C} \\ \text { HETATM } & 16 & \mathrm{H} & 0 & -2.400 & -2.001 & 0.032 & \mathrm{H} \\ \text { HETATM } & 17 & \mathrm{H} & 0 & -1.306 & -1.920 & -1.324 & \mathrm{H} \\ \text { HETATM } & 18 & \mathrm{C} & 0 & -1.746 & -0.010 & -0.477 & \mathrm{C} \\ \text { HETATM } & 19 & \mathrm{H} & 0 & -0.922 & 0.540 & -0.931 & \mathrm{H} \\ \text { HETATM } & 20 & \mathrm{C} & 0 & -2.822 & 0.699 & -0.111 & \mathrm{C} \\ \text { HETATM } & 21 & \mathrm{C} & 0 & -2.875 & 2.191 & -0.336 & \mathrm{C} \\ \text { HETATM } & 22 & \mathrm{H} & 0 & -3.725 & 2.464 & -0.973 & \mathrm{H} \\ \text { HETATM } & 23 & \mathrm{H} & 0 & -3.015 & 2.728 & 0.610 & \mathrm{H} \\ \text { HETATM } & 24 & \mathrm{H} & 0 & -1.965 & 2.568 & -0.807 & \mathrm{H} \\ \text { HETATM } & 25 & \mathrm{C} & 0 & -4.059 & 0.128 & 0.537 & \mathrm{C} \\ \text { HETATM } & 26 & \mathrm{H} & 0 & -4.004 & -0.945 & 0.717 & \mathrm{H} \\ \text { HETATM } & 27 & \mathrm{H} & 0 & -4.250 & 0.618 & 1.499 & \mathrm{H} \\ \text { HETATM } & 28 & \mathrm{H} & 0 & -4.942 & 0.318 & -0.084 & \mathrm{H}\end{array}$

Geranyl Chloride Down Conformation

$\begin{array}{llllrrrr}\text { HETATM } & 1 & \mathrm{C} & 0 & -1.857 & -0.476 & 0.125 & \mathrm{C} \\ \text { HETATM } & 2 & \mathrm{H} & 0 & -1.476 & -1.414 & -0.275 & \mathrm{H} \\ \text { HETATM } & 3 & \mathrm{C} & 0 & -1.082 & 0.617 & 0.036 & \mathrm{C} \\ \text { HETATM } & 4 & \mathrm{C} & 0 & 0.300 & 0.510 & -0.569 & \mathrm{C} \\ \text { HETATM } & 5 & \mathrm{H} & 0 & 0.420 & -0.464 & -1.050 & \mathrm{H} \\ \text { HETATM } & 6 & \mathrm{H} & 0 & 0.406 & 1.267 & -1.357 & \mathrm{H} \\ \text { HETATM } & 7 & \mathrm{C} & 0 & -1.486 & 1.990 & 0.510 & \mathrm{C} \\ \text { HETATM } & 8 & \mathrm{H} & 0 & -0.905 & 2.295 & 1.386 & \mathrm{H} \\ \text { HETATM } & 9 & \mathrm{H} & 0 & -1.282 & 2.731 & -0.270 & \mathrm{H} \\ \text { HETATM } & 10 & \mathrm{H} & 0 & -2.541 & 2.068 & 0.771 & \mathrm{H} \\ \text { HETATM } & 11 & \mathrm{C} & 0 & 1.448 & 0.700 & 0.455 & \mathrm{C} \\ \text { HETATM } & 12 & \mathrm{H} & 0 & 1.347 & -0.051 & 1.242 & \mathrm{H} \\ \text { HETATM } & 13 & \mathrm{H} & 0 & 1.335 & 1.676 & 0.943 & \mathrm{H} \\ \text { HETATM } & 14 & \mathrm{C} & 0 & 2.800 & 0.643 & -0.202 & \mathrm{C} \\ \text { HETATM } & 15 & \mathrm{H} & 0 & 2.995 & 1.462 & -0.895 & \mathrm{H} \\ \text { HETATM } & 16 & \mathrm{C} & 0 & 3.763 & -0.278 & -0.056 & \mathrm{C} \\ \text { HETATM } & 17 & \mathrm{C} & 0 & 5.062 & -0.165 & -0.816 & \mathrm{C} \\ \text { HETATM } & 18 & \mathrm{H} & 0 & 5.917 & -0.114 & -0.131 & \mathrm{H} \\ \text { HETATM } & 19 & \mathrm{H} & 0 & 5.227 & -1.046 & -1.448 & \mathrm{H} \\ \text { HETATM } & 20 & \mathrm{H} & 0 & 5.089 & 0.720 & -1.456 & \mathrm{H} \\ \text { HETATM } & 21 & \mathrm{C} & 0 & 3.676 & -1.483 & 0.846 & \mathrm{C} \\ \text { HETATM } & 22 & \mathrm{H} & 0 & 4.472 & -1.461 & 1.600 & \mathrm{H} \\ \text { HETATM } & 23 & \mathrm{H} & 0 & 2.724 & -1.568 & 1.370 & \mathrm{H} \\ \text { HETATM } & 24 & \mathrm{H} & 0 & 3.824 & -2.405 & 0.272 & \mathrm{H} \\ \text { HETATM } & 25 & \mathrm{C} & 0 & -3.204 & -0.557 & 0.734 & \mathrm{C}\end{array}$


$\begin{array}{lrllrrrr}\text { HETATM } & 26 \mathrm{H} & 0 & -3.343 & -1.464 & 1.316 & \mathrm{H} \\ \text { HETATM } & 27 \mathrm{H} & 0 & -3.487 & 0.305 & 1.329 & \mathrm{H} \\ \text { HETATM } & 28 \mathrm{Cl} & 0 & -4.544 & -0.679 & -0.572 & \mathrm{Cl}\end{array}$

Geranyl Chloride Far Down Conformation

$\begin{array}{llllrrrr}\text { HETATM } & 1 & \mathrm{C} & 0 & -2.398 & -1.055 & 0.180 & \mathrm{C} \\ \text { HETATM } & 2 & \mathrm{H} & 0 & -2.378 & -2.065 & -0.221 & \mathrm{H} \\ \text { HETATM } & 3 & \mathrm{H} & 0 & -2.237 & -1.086 & 1.252 & \mathrm{H} \\ \text { HETATM } & 4 & \mathrm{Cl} & 0 & -4.198 & -0.558 & -0.004 & \mathrm{Cl} \\ \text { HETATM } & 5 & \mathrm{C} & 0 & -1.501 & -0.137 & -0.557 & \mathrm{C} \\ \text { HETATM } & 6 & \mathrm{H} & 0 & -1.480 & -0.281 & -1.635 & \mathrm{H} \\ \text { HETATM } & 7 & \mathrm{C} & 0 & -0.725 & 0.821 & -0.024 & \mathrm{C} \\ \text { HETATM } & 8 & \mathrm{C} & 0 & 0.166 & 1.656 & -0.915 & \mathrm{C} \\ \text { HETATM } & 9 & \mathrm{H} & 0 & -0.010 & 1.390 & -1.962 & \mathrm{H} \\ \text { HETATM } & 10 & \mathrm{H} & 0 & -0.121 & 2.710 & -0.808 & \mathrm{H} \\ \text { HETATM } & 11 & \mathrm{C} & 0 & -0.673 & 1.154 & 1.445 & \mathrm{C} \\ \text { HETATM } & 12 & \mathrm{H} & 0 & -1.478 & 0.701 & 2.022 & \mathrm{H} \\ \text { HETATM } & 13 & \mathrm{H} & 0 & 0.275 & 0.830 & 1.887 & \mathrm{H} \\ \text { HETATM } & 14 & \mathrm{H} & 0 & -0.730 & 2.238 & 1.588 & \mathrm{H} \\ \text { HETATM } & 15 & \mathrm{C} & 0 & 1.681 & 1.542 & -0.614 & \mathrm{C} \\ \text { HETATM } & 16 & \mathrm{H} & 0 & 1.888 & 1.898 & 0.397 & \mathrm{H} \\ \text { HETATM } & 17 & \mathrm{H} & 0 & 2.193 & 2.243 & -1.286 & \mathrm{H} \\ \text { HETATM } & 18 & \mathrm{C} & 0 & 2.222 & 0.156 & -0.839 & \mathrm{C} \\ \text { HETATM } & 19 & \mathrm{H} & 0 & 1.953 & -0.275 & -1.804 & \mathrm{H} \\ \text { HETATM } & 20 & \mathrm{C} & 0 & 2.982 & -0.592 & -0.029 & \mathrm{C} \\ \text { HETATM } & 21 & \mathrm{C} & 0 & 3.436 & -1.969 & -0.450 & \mathrm{C} \\ \text { HETATM } & 22 & \mathrm{H} & 0 & 3.067 & -2.236 & -1.443 & \mathrm{H} \\ \text { HETATM } & 23 & \mathrm{H} & 0 & 4.530 & -2.038 & -0.465 & \mathrm{H} \\ \text { HETATM } & 24 & \mathrm{H} & 0 & 3.089 & -2.732 & 0.257 & \mathrm{H} \\ \text { HETATM } & 25 & \mathrm{C} & 0 & 3.470 & -0.171 & 1.335 & \mathrm{C} \\ \text { HETATM } & 26 & \mathrm{H} & 0 & 4.566 & -0.164 & 1.366 & \mathrm{H} \\ \text { HETATM } & 27 & \mathrm{H} & 0 & 3.126 & 0.818 & 1.637 & \mathrm{H} \\ \text { HETATM } & 28 & \mathrm{H} & 0 & 3.148 & -0.888 & 2.100 & \mathrm{H} \\ & & & & & & & \end{array}$

Pyridine

$\begin{array}{llllrrrr}\text { HETATM } & 1 & \mathrm{C} & 0 & -1.143 & 0.721 & 0.000 & \mathrm{C} \\ \text { HETATM } & 2 & \mathrm{C} & 0 & -1.197 & -0.672 & -0.000 & \mathrm{C} \\ \text { HETATM } & 3 & \mathrm{C} & 0 & 0.000 & -1.383 & -0.000 & \mathrm{C} \\ \text { HETATM } & 4 & \mathrm{C} & 0 & 1.197 & -0.672 & -0.000 & \mathrm{C} \\ \text { HETATM } & 5 & \mathrm{C} & 0 & 1.143 & 0.721 & 0.000 & \mathrm{C} \\ \text { HETATM } & 6 & \mathrm{~N} & 0 & -0.000 & 1.419 & 0.000 & \mathrm{~N} \\ \text { HETATM } & 7 & \mathrm{H} & 0 & 0.000 & -2.468 & 0.000 & \mathrm{H} \\ \text { HETATM } & 8 & \mathrm{H} & 0 & -2.059 & 1.305 & 0.000 & \mathrm{H} \\ \text { HETATM } & 9 & \mathrm{H} & 0 & -2.155 & -1.180 & -0.000 & \mathrm{H} \\ \text { HETATM } & 10 & \mathrm{H} & 0 & 2.155 & -1.180 & -0.000 & \mathrm{H} \\ \text { HETATM } & 11 & \mathrm{H} & 0 & 2.059 & 1.305 & 0.000 & \mathrm{H}\end{array}$




\subsection{Refined Transition-State Geometries}

Prenyl Chloride

\begin{tabular}{|c|c|c|c|c|c|c|}
\hline HETATM & $1 \mathrm{C}$ & 0 & -0.870 & -0.810 & -0.204 & $\mathrm{C}$ \\
\hline HETATM & $2 \mathrm{H}$ & 0 & -0.579 & -1.583 & -0.891 & $\mathrm{H}$ \\
\hline HETATM & $3 \mathrm{H}$ & 0 & -0.792 & -1.034 & 0.844 & $\mathrm{H}$ \\
\hline HETATM & $4 \mathrm{C}$ & 0 & 2.198 & -1.376 & 0.184 & $\mathrm{C}$ \\
\hline HETATM & $5 \mathrm{C}$ & 0 & 3.575 & -1.191 & 0.264 & $\mathrm{C}$ \\
\hline HETATM & $6 \mathrm{C}$ & 0 & 4.091 & 0.084 & 0.039 & $\mathrm{C}$ \\
\hline HETATM & $7 \mathrm{C}$ & 0 & 3.213 & 1.124 & -0.257 & $\mathrm{C}$ \\
\hline HETATM & $8 \mathrm{C}$ & 0 & 1.850 & 0.848 & -0.316 & $\mathrm{C}$ \\
\hline HETATM & $9 \mathrm{~N}$ & 0 & 1.360 & -0.374 & -0.100 & $\mathrm{~N}$ \\
\hline HETATM & $10 \mathrm{H}$ & 0 & 5.159 & 0.263 & 0.092 & $\mathrm{H}$ \\
\hline HETATM & $11 \mathrm{H}$ & 0 & 1.751 & -2.351 & 0.352 & $\mathrm{H}$ \\
\hline HETATM & $12 \mathrm{H}$ & 0 & 4.222 & -2.029 & 0.497 & $\mathrm{H}$ \\
\hline HETATM & $13 \mathrm{H}$ & 0 & 3.571 & 2.131 & -0.439 & $\mathrm{H}$ \\
\hline HETATM & $14 \mathrm{H}$ & 0 & 1.122 & 1.622 & -0.543 & $\mathrm{H}$ \\
\hline HETATM & $15 \mathrm{Cl}$ & 0 & -3.011 & -2.215 & -0.090 & $\mathrm{Cl}$ \\
\hline HETATM & $16 \mathrm{C}$ & 0 & -1.374 & 0.432 & -0.695 & $\mathrm{C}$ \\
\hline HETATM & $17 \mathrm{H}$ & 0 & -1.440 & 0.526 & -1.775 & $\mathrm{H}$ \\
\hline HETATM & $18 \mathrm{C}$ & 0 & -1.770 & 1.483 & 0.068 & $\mathrm{C}$ \\
\hline HETATM & $19 \mathrm{C}$ & 0 & -1.754 & 1.498 & 1.566 & $\mathrm{C}$ \\
\hline HETATM & $20 \mathrm{H}$ & 0 & -1.104 & 2.303 & 1.926 & $\mathrm{H}$ \\
\hline HETATM & $21 \mathrm{H}$ & 0 & -2.756 & 1.723 & 1.945 & $\mathrm{H}$ \\
\hline HETATM & $22 \mathrm{H}$ & 0 & -1.423 & 0.565 & 2.018 & $\mathrm{H}$ \\
\hline HETATM & $23 \mathrm{C}$ & 0 & -2.287 & 2.732 & -0.581 & $\mathrm{C}$ \\
\hline HETATM & $24 \mathrm{H}$ & 0 & -3.330 & 2.904 & -0.290 & $\mathrm{H}$ \\
\hline HETATM & $25 \mathrm{H}$ & 0 & -1.727 & 3.609 & -0.238 & $\mathrm{H}$ \\
\hline HETATM & $26 \mathrm{H}$ & 0 & -2.237 & 2.688 & -1.669 & $\mathrm{H}$ \\
\hline
\end{tabular}

Geranyl Chloride Far Up Conformation

$\begin{array}{llllllll}\text { HETATM } & 1 & \mathrm{C} & 0 & -0.940 & 1.188 & -0.196 & \mathrm{C} \\ \text { HETATM } & 2 & \mathrm{H} & 0 & -1.407 & 1.884 & -0.867 & \mathrm{H} \\ \text { HETATM } & 3 & \mathrm{H} & 0 & -1.061 & 1.370 & 0.856 & \mathrm{H} \\ \text { HETATM } & 4 & \mathrm{C} & 0 & -4.063 & 1.047 & 0.222 & \mathrm{C} \\ \text { HETATM } & 5 & \mathrm{C} & 0 & -5.363 & 0.559 & 0.306 & \mathrm{C} \\ \text { HETATM } & 6 & \mathrm{C} & 0 & -5.583 & -0.796 & 0.070 & \mathrm{C} \\ \text { HETATM } & 7 & \mathrm{C} & 0 & -4.498 & -1.611 & -0.241 & \mathrm{C} \\ \text { HETATM } & 8 & \mathrm{C} & 0 & -3.232 & -1.038 & -0.303 & \mathrm{C} \\ \text { HETATM } & 9 & \mathrm{~N} & 0 & -3.025 & 0.260 & -0.076 & \mathrm{~N} \\ \text { HETATM } & 10 & \mathrm{H} & 0 & -6.584 & -1.209 & 0.126 & \mathrm{H} \\ \text { HETATM } & 11 & \mathrm{H} & 0 & -3.843 & 2.095 & 0.399 & \mathrm{H} \\ \text { HETATM } & 12 & \mathrm{H} & 0 & -6.179 & 1.229 & 0.552 & \mathrm{H} \\ \text { HETATM } & 13 & \mathrm{H} & 0 & -4.625 & -2.670 & -0.432 & \mathrm{H} \\ \text { HETATM } & 14 & \mathrm{H} & 0 & -2.351 & -1.628 & -0.541 & \mathrm{H} \\ \text { HETATM } & 15 & \mathrm{Cl} & 0 & 0.782 & 3.085 & -0.053 & \mathrm{Cl} \\ \text { HETATM } & 16 & \mathrm{C} & 0 & -0.179 & 0.097 & -0.710 & \mathrm{C} \\ \text { HETATM } & 17 & \mathrm{H} & 0 & -0.107 & 0.031 & -1.791 & \mathrm{H} \\ \text { HETATM } & 18 & \mathrm{C} & 0 & 0.434 & -0.860 & 0.036 & \mathrm{C} \\ \text { HETATM } & 19 & \mathrm{C} & 0 & 1.152 & -1.997 & -0.645 & \mathrm{C} \\ \text { HETATM } & 20 & \mathrm{C} & 0 & 0.449 & -0.867 & 1.535 & \mathrm{C} \\ \text { HETATM } & 21 \mathrm{C} & 0 & 2.650 & -2.146 & -0.277 & \mathrm{C} \\ \text { HETATM } & 22 & \mathrm{C} & 0 & 3.485 & -0.982 & -0.737 & \mathrm{C}\end{array}$




$\begin{array}{llllrrrrr}\text { HETATM } & 23 & \mathrm{C} & 0 & 4.377 & -0.265 & -0.042 & \mathrm{C} \\ \text { HETATM } & 24 & \mathrm{C} & 0 & 5.122 & 0.876 & -0.692 & \mathrm{C} \\ \text { HETATM } & 25 & \mathrm{C} & 0 & 4.739 & -0.496 & 1.403 & \mathrm{C} \\ \text { HETATM } & 26 & \mathrm{H} & 0 & 1.406 & -0.474 & 1.894 & \mathrm{H} \\ \text { HETATM } & 27 & \mathrm{H} & 0 & 0.645 & -2.930 & -0.364 & \mathrm{H} \\ \text { HETATM } & 28 & \mathrm{H} & 0 & -0.338 & -0.261 & 1.980 & \mathrm{H} \\ \text { HETATM } & 29 & \mathrm{H} & 0 & 0.359 & -1.886 & 1.920 & \mathrm{H} \\ \text { HETATM } & 30 & \mathrm{H} & 0 & 1.054 & -1.901 & -1.729 & \mathrm{H} \\ \text { HETATM } & 31 & \mathrm{H} & 0 & 3.000 & -3.063 & -0.768 & \mathrm{H} \\ \text { HETATM } & 32 & \mathrm{H} & 0 & 2.755 & -2.324 & 0.795 & \mathrm{H} \\ \text { HETATM } & 33 & \mathrm{H} & 0 & 3.328 & -0.707 & -1.780 & \mathrm{H} \\ \text { HETATM } & 34 & \mathrm{H} & 0 & 5.806 & -0.731 & 1.499 & \mathrm{H} \\ \text { HETATM } & 35 & \mathrm{H} & 0 & 4.571 & 0.412 & 1.993 & \mathrm{H} \\ \text { HETATM } & 36 & \mathrm{H} & 0 & 4.181 & -1.308 & 1.871 & \mathrm{H} \\ \text { HETATM } & 37 & \mathrm{H} & 0 & 6.206 & 0.721 & -0.640 & \mathrm{H} \\ \text { HETATM } & 38 & \mathrm{H} & 0 & 4.846 & 1.001 & -1.740 & \mathrm{H} \\ \text { HETATM } & 39 & \mathrm{H} & 0 & 4.917 & 1.821 & -0.175 & \mathrm{H}\end{array}$

Geranyl Chloride Up Conformation

$\begin{array}{llllrrrr}\text { HETATM } & 1 & \mathrm{C} & 0 & -1.102 & 1.230 & -0.052 & \mathrm{C} \\ \text { HETATM } & 2 & \mathrm{H} & 0 & -1.465 & 1.813 & -0.878 & \mathrm{H} \\ \text { HETATM } & 3 & \mathrm{H} & 0 & -1.455 & 1.497 & 0.927 & \mathrm{H} \\ \text { HETATM } & 4 & \mathrm{C} & 0 & -4.224 & 0.826 & -0.287 & \mathrm{C} \\ \text { HETATM } & 5 & \mathrm{C} & 0 & -5.471 & 0.223 & -0.408 & \mathrm{C} \\ \text { HETATM } & 6 & \mathrm{C} & 0 & -5.533 & -1.166 & -0.506 & \mathrm{C} \\ \text { HETATM } & 7 & \mathrm{C} & 0 & -4.349 & -1.897 & -0.479 & \mathrm{C} \\ \text { HETATM } & 8 & \mathrm{C} & 0 & -3.145 & -1.209 & -0.355 & \mathrm{C} \\ \text { HETATM } & 9 & \mathrm{~N} & 0 & -3.090 & 0.120 & -0.262 & \mathrm{~N} \\ \text { HETATM } & 10 & \mathrm{H} & 0 & -6.489 & -1.669 & -0.602 & \mathrm{H} \\ \text { HETATM } & 11 & \mathrm{H} & 0 & -4.126 & 1.905 & -0.208 & \mathrm{H} \\ \text { HETATM } & 12 & \mathrm{H} & 0 & -6.368 & 0.830 & -0.425 & \mathrm{H} \\ \text { HETATM } & 13 & \mathrm{H} & 0 & -4.351 & -2.978 & -0.552 & \mathrm{H} \\ \text { HETATM } & 14 & \mathrm{H} & 0 & -2.193 & -1.731 & -0.330 & \mathrm{H} \\ \text { HETATM } & 15 & \mathrm{Cl} & 0 & 0.449 & 3.236 & 0.249 & \mathrm{Cl} \\ \text { HETATM } & 16 & \mathrm{C} & 0 & -0.178 & 0.166 & -0.276 & \mathrm{C} \\ \text { HETATM } & 17 & \mathrm{H} & 0 & 0.107 & -0.011 & -1.309 & \mathrm{H} \\ \text { HETATM } & 18 & \mathrm{C} & 0 & 0.364 & -0.624 & 0.687 & \mathrm{C} \\ \text { HETATM } & 19 & \mathrm{C} & 0 & 1.324 & -1.721 & 0.311 & \mathrm{C} \\ \text { HETATM } & 20 & \mathrm{H} & 0 & 0.949 & -2.661 & 0.734 & \mathrm{H} \\ \text { HETATM } & 21 & \mathrm{H} & 0 & 1.350 & -1.843 & -0.775 & \mathrm{H} \\ \text { HETATM } & 22 & \mathrm{C} & 0 & 0.088 & -0.453 & 2.152 & \mathrm{C} \\ \text { HETATM } & 23 & \mathrm{H} & 0 & 0.064 & -1.421 & 2.659 & \mathrm{H} \\ \text { HETATM } & 24 & \mathrm{H} & 0 & 0.890 & 0.129 & 2.621 & \mathrm{H} \\ \text { HETATM } & 25 & \mathrm{H} & 0 & -0.848 & 0.064 & 2.359 & \mathrm{H} \\ \text { HETATM } & 26 & \mathrm{C} & 0 & 2.775 & -1.516 & 0.831 & \mathrm{C} \\ \text { HETATM } & 27 & \mathrm{H} & 0 & 3.318 & -2.448 & 0.664 & \mathrm{H} \\ \text { HETATM } & 28 & \mathrm{H} & 0 & 2.744 & -1.378 & 1.919 & \mathrm{H} \\ \text { HETATM } & 29 & \mathrm{C} & 0 & 3.475 & -0.349 & 0.191 & \mathrm{C} \\ \text { HETATM } & 30 & \mathrm{H} & 0 & 2.968 & 0.608 & 0.314 & \mathrm{H} \\ \text { HETATM } & 31 & \mathrm{C} & 0 & 4.622 & -0.346 & -0.501 & \mathrm{C} \\ \text { HETATM } & 32 & \mathrm{C} & 0 & 5.176 & 0.938 & -1.068 & \mathrm{C} \\ \text { HETATM } & 33 & \mathrm{H} & 0 & 6.175 & 1.147 & -0.668 & \mathrm{H} \\ \text { HETATM } & 34 & \mathrm{H} & 0 & 5.288 & 0.872 & -2.157 & \\ \text { HETATM } & 35 & \mathrm{H} & 0 & 4.537 & 1.795 & -0.846 & \mathrm{H} \\ & & & & & & & \end{array}$




$\begin{array}{llllllll}\text { HETATM } & 36 & \mathrm{C} & 0 & 5.471 & -1.561 & -0.781 & \mathrm{C} \\ \text { HETATM } & 37 & \mathrm{H} & 0 & 5.048 & -2.490 & -0.399 & \mathrm{H} \\ \text { HETATM } & 38 & \mathrm{H} & 0 & 5.626 & -1.682 & -1.859 & \mathrm{H} \\ \text { HETATM } & 39 & \mathrm{H} & 0 & 6.468 & -1.444 & -0.340 & \mathrm{H}\end{array}$

Geranyl Chloride Down Conformation

\begin{tabular}{|c|c|c|c|c|c|c|}
\hline HETATM & $1 \mathrm{C}$ & 0 & -1.995 & 1.320 & -0.387 & $\mathrm{C}$ \\
\hline HETATM & $2 \mathrm{H}$ & 0 & -2.590 & 1.242 & -1.279 & $\mathrm{H}$ \\
\hline HETATM & $3 \mathrm{H}$ & 0 & -2.522 & 1.442 & 0.541 & $\mathrm{H}$ \\
\hline HETATM & $4 \mathrm{C}$ & 0 & -3.819 & -1.256 & -0.190 & $\mathrm{C}$ \\
\hline HETATM & $5 \mathrm{C}$ & 0 & -4.235 & -2.576 & -0.049 & $\mathrm{C}$ \\
\hline HETATM & $6 \mathrm{C}$ & 0 & -3.270 & -3.561 & 0.150 & $\mathrm{C}$ \\
\hline HETATM & $7 \mathrm{C}$ & 0 & -1.929 & -3.191 & 0.201 & $\mathrm{C}$ \\
\hline HETATM & $8 \mathrm{C}$ & 0 & -1.605 & -1.845 & 0.050 & $\mathrm{C}$ \\
\hline HETATM & $9 \mathrm{~N}$ & 0 & -2.530 & -0.905 & -0.141 & $\mathrm{~N}$ \\
\hline HETATM & $10 \mathrm{H}$ & 0 & -3.559 & -4.600 & 0.264 & $\mathrm{H}$ \\
\hline HETATM & $11 \mathrm{H}$ & 0 & -4.535 & -0.455 & -0.347 & $\mathrm{H}$ \\
\hline HETATM & $12 \mathrm{H}$ & 0 & -5.289 & -2.821 & -0.094 & $\mathrm{H}$ \\
\hline HETATM & $13 \mathrm{H}$ & 0 & -1.146 & -3.924 & 0.354 & $\mathrm{H}$ \\
\hline HETATM & $14 \mathrm{H}$ & 0 & -0.574 & -1.503 & 0.081 & $\mathrm{H}$ \\
\hline HETATM & $15 \mathrm{Cl}$ & 0 & -2.475 & 3.811 & -0.704 & $\mathrm{Cl}$ \\
\hline HETATM & $16 \mathrm{C}$ & 0 & -0.573 & 1.280 & -0.468 & $\mathrm{C}$ \\
\hline HETATM & $17 \mathrm{H}$ & 0 & -0.152 & 1.201 & -1.467 & $\mathrm{H}$ \\
\hline HETATM & $18 \mathrm{C}$ & 0 & 0.283 & 1.361 & 0.585 & $\mathrm{C}$ \\
\hline HETATM & $19 \mathrm{C}$ & 0 & 1.769 & 1.336 & 0.345 & $\mathrm{C}$ \\
\hline HETATM & $20 \mathrm{H}$ & 0 & 1.985 & 1.564 & -0.702 & $\mathrm{H}$ \\
\hline HETATM & $21 \mathrm{H}$ & 0 & 2.239 & 2.121 & 0.949 & $\mathrm{H}$ \\
\hline HETATM & $22 \mathrm{C}$ & 0 & -0.154 & 1.517 & 2.010 & $\mathrm{C}$ \\
\hline HETATM & $23 \mathrm{H}$ & 0 & 0.424 & 0.866 & 2.671 & $\mathrm{H}$ \\
\hline HETATM & $24 \mathrm{H}$ & 0 & 0.045 & 2.544 & 2.337 & $\mathrm{H}$ \\
\hline HETATM & $25 \mathrm{H}$ & 0 & -1.211 & 1.314 & 2.173 & $\mathrm{H}$ \\
\hline HETATM & $26 \mathrm{C}$ & 0 & 2.432 & -0.022 & 0.704 & $\mathrm{C}$ \\
\hline HETATM & $27 \mathrm{H}$ & 0 & 1.999 & -0.805 & 0.078 & $\mathrm{H}$ \\
\hline HETATM & $28 \mathrm{H}$ & 0 & 2.182 & -0.279 & 1.740 & $\mathrm{H}$ \\
\hline HETATM & $29 \mathrm{C}$ & 0 & 3.929 & 0.034 & 0.564 & $\mathrm{C}$ \\
\hline HETATM & $30 \mathrm{H}$ & 0 & 4.415 & 0.731 & 1.248 & $\mathrm{H}$ \\
\hline HETATM & $31 \mathrm{C}$ & 0 & 4.713 & -0.646 & -0.282 & C \\
\hline HETATM & $32 \mathrm{C}$ & 0 & 6.210 & -0.454 & -0.268 & C \\
\hline HETATM & $33 \mathrm{H}$ & 0 & 6.726 & -1.400 & -0.065 & $\mathrm{H}$ \\
\hline HETATM & $34 \mathrm{H}$ & 0 & 6.572 & -0.110 & -1.244 & $\mathrm{H}$ \\
\hline HETATM & $35 \mathrm{H}$ & 0 & 6.525 & 0.272 & 0.485 & $\mathrm{H}$ \\
\hline HETATM & $36 \mathrm{C}$ & 0 & 4.227 & -1.645 & -1.301 & $\mathrm{C}$ \\
\hline HETATM & $37 \mathrm{H}$ & 0 & 4.653 & -2.636 & -1.103 & $\mathrm{H}$ \\
\hline HETATM & $38 \mathrm{H}$ & 0 & 3.143 & -1.750 & -1.330 & $\mathrm{H}$ \\
\hline HETATM & $39 \mathrm{H}$ & 0 & 4.562 & -1.363 & -2.306 & \\
\hline
\end{tabular}

Geranyl Chloride Far Down Conformation

$\begin{array}{llllllll}\text { HETATM } & 1 & \mathrm{C} & 0 & 2.261 & -0.543 & -0.144 & \mathrm{C} \\ \text { HETATM } & 2 & \mathrm{H} & 0 & 2.998 & -0.090 & -0.781 & \mathrm{H} \\ \text { HETATM } & 3 & \mathrm{H} & 0 & 2.397 & -0.430 & 0.916 & \mathrm{H} \\ \text { HETATM } & 4 & \mathrm{C} & 0 & 2.298 & 2.594 & 0.244 & \mathrm{C} \\ \text { HETATM } & 5 & \mathrm{C} & 0 & 1.910 & 3.929 & 0.287 & \mathrm{C}\end{array}$




$\begin{array}{lccccccc}\text { HETATM } & 6 & \mathrm{C} & 0 & 0.583 & 4.246 & 0.009 & \mathrm{C} \\ \text { HETATM } & 7 & \mathrm{C} & 0 & -0.306 & 3.219 & -0.298 & \mathrm{C} \\ \text { HETATM } & 8 & \mathrm{C} & 0 & 0.169 & 1.910 & -0.316 & \mathrm{C} \\ \text { HETATM } & 9 & \mathrm{~N} & 0 & 1.441 & 1.610 & -0.052 & \mathrm{~N} \\ \text { HETATM } & 10 & \mathrm{H} & 0 & 0.246 & 5.276 & 0.032 & \mathrm{H} \\ \text { HETATM } & 11 & \mathrm{H} & 0 & 3.322 & 2.299 & 0.450 & \mathrm{H} \\ \text { HETATM } & 12 & \mathrm{H} & 0 & 2.635 & 4.697 & 0.531 & \mathrm{H} \\ \text { HETATM } & 13 & \mathrm{H} & 0 & -1.346 & 3.422 & -0.521 & \mathrm{H} \\ \text { HETATM } & 14 & \mathrm{H} & 0 & -0.485 & 1.072 & -0.545 & \mathrm{H} \\ \text { HETATM } & 15 & \mathrm{Cl} & 0 & 4.023 & -2.367 & 0.043 & \mathrm{Cl} \\ \text { HETATM } & 16 & \mathrm{C} & 0 & 1.162 & -1.252 & -0.713 & \mathrm{C} \\ \text { HETATM } & 17 & \mathrm{H} & 0 & 1.147 & -1.313 & -1.797 & \mathrm{H} \\ \text { HETATM } & 18 & \mathrm{C} & 0 & 0.172 & -1.873 & -0.021 & \mathrm{C} \\ \text { HETATM } & 19 & \mathrm{C} & 0 & -0.870 & -2.669 & -0.765 & \mathrm{C} \\ \text { HETATM } & 20 & \mathrm{H} & 0 & -0.741 & -2.531 & -1.842 & \mathrm{H} \\ \text { HETATM } & 21 & \mathrm{H} & 0 & -0.665 & -3.730 & -0.564 & \mathrm{H} \\ \text { HETATM } & 22 & \mathrm{C} & 0 & 0.073 & -1.881 & 1.475 & \mathrm{C} \\ \text { HETATM } & 23 & \mathrm{H} & 0 & 0.969 & -1.516 & 1.972 & \mathrm{H} \\ \text { HETATM } & 24 & \mathrm{H} & 0 & -0.769 & -1.262 & 1.801 & \mathrm{H} \\ \text { HETATM } & 25 & \mathrm{H} & 0 & -0.128 & -2.894 & 1.837 & \mathrm{H} \\ \text { HETATM } & 26 & \mathrm{C} & 0 & -2.343 & -2.395 & -0.380 & \mathrm{C} \\ \text { HETATM } & 27 & \mathrm{H} & 0 & -2.498 & -2.617 & 0.678 & \mathrm{H} \\ \text { HETATM } & 28 & \mathrm{H} & 0 & -2.948 & -3.129 & -0.928 & \mathrm{H} \\ \text { HETATM } & 29 & \mathrm{C} & 0 & -2.809 & -1.009 & -0.737 & \mathrm{C} \\ \text { HETATM } & 30 & \mathrm{H} & 0 & -2.538 & -0.692 & -1.744 & \mathrm{H} \\ \text { HETATM } & 31 & \mathrm{C} & 0 & -3.536 & -0.157 & -0.001 & \mathrm{C} \\ \text { HETATM } & 32 & \mathrm{C} & 0 & -3.955 & 1.182 & -0.557 & \mathrm{C} \\ \text { HETATM } & 33 & \mathrm{H} & 0 & -3.568 & 1.349 & -1.564 & \mathrm{H} \\ \text { HETATM } & 34 & \mathrm{H} & 0 & -5.048 & 1.264 & -0.597 & \mathrm{H} \\ \text { HETATM } & 35 & \mathrm{H} & 0 & -3.610 & 2.002 & 0.083 & \mathrm{H} \\ \text { HETATM } & 36 & \mathrm{C} & 0 & -4.031 & -0.426 & 1.398 & \mathrm{C} \\ \text { HETATM } & 37 & \mathrm{H} & 0 & -5.127 & -0.411 & 1.425 & \mathrm{H} \\ \text { HETATM } & 38 & \mathrm{H} & 0 & -3.707 & -1.385 & 1.802 & \mathrm{H} \\ \text { HETATM } & 39 & \mathrm{H} & 0 & -3.697 & 0.359 & 2.085 & \end{array}$

